\title{
Fixation of Dinitrogen at an Asymmetric Binuclear Titanium Complex
}

Dae Young Bae, Gunhee Lee, and Eunsung Lee*

Department of Chemistry, Pohang University of Science and Technology (POSTECH), Pohang, 37673, Republic of Korea 


\section{Table of contents}

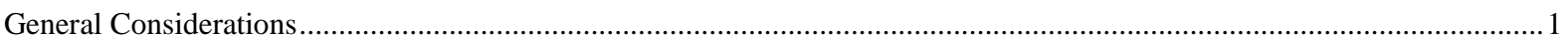

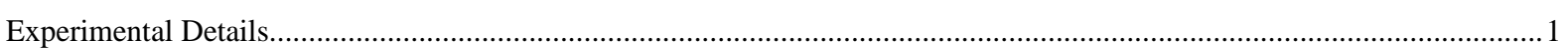

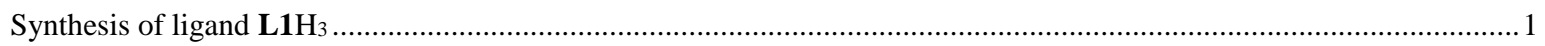

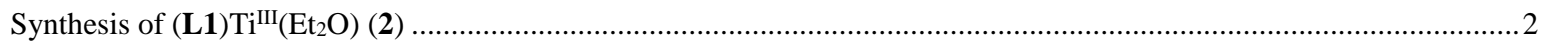

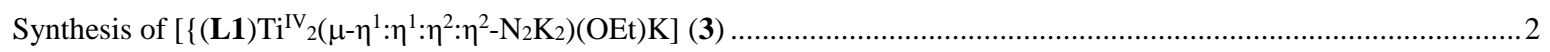

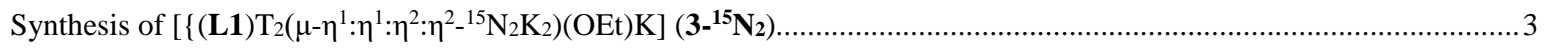

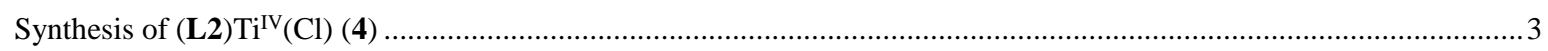

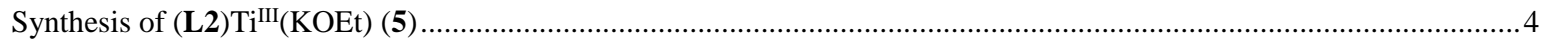

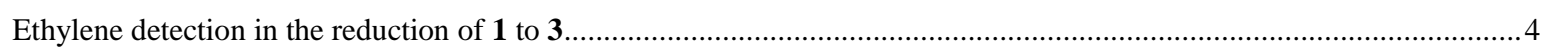

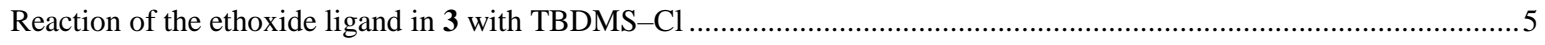

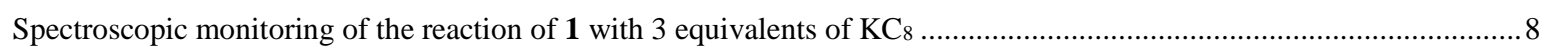

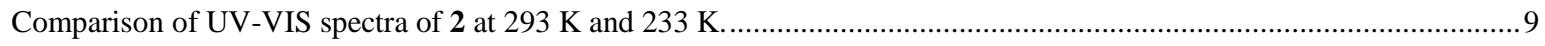

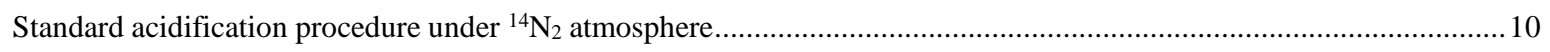

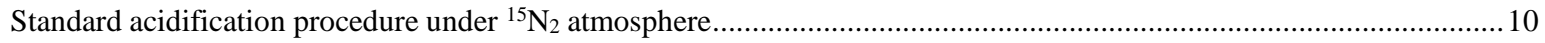

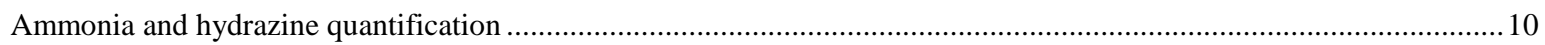

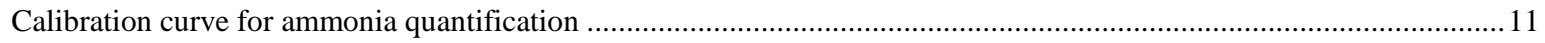

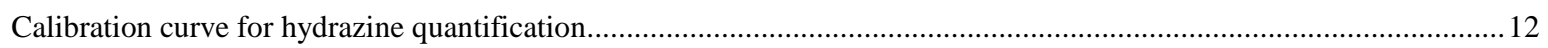

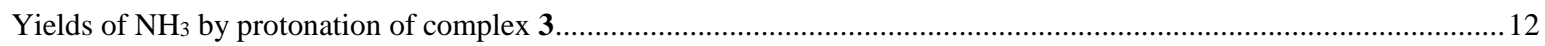

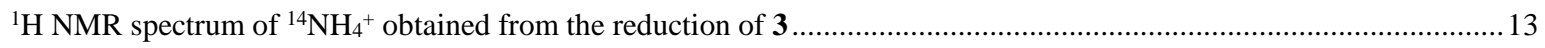

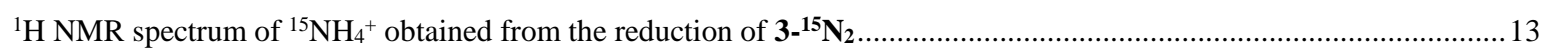

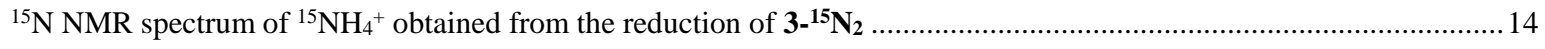

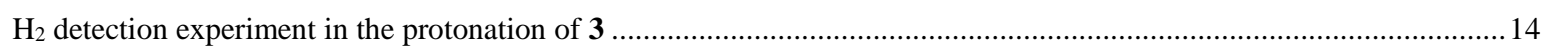

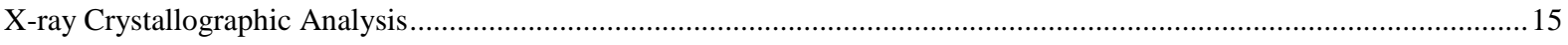

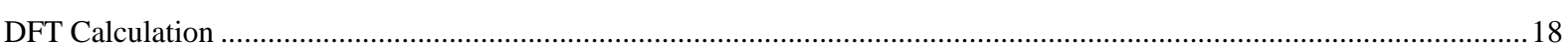

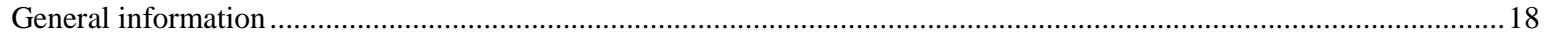

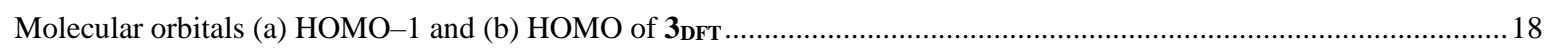

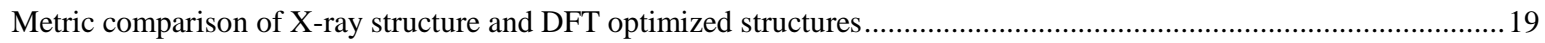

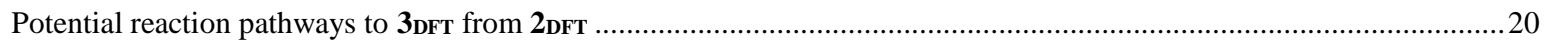

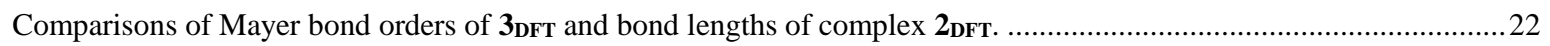

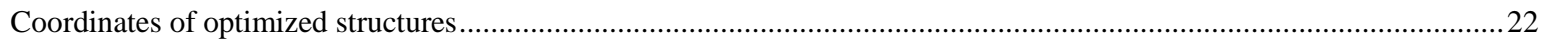

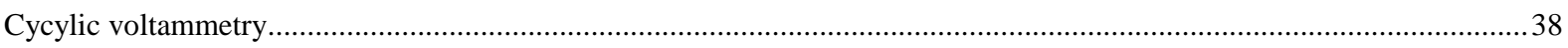

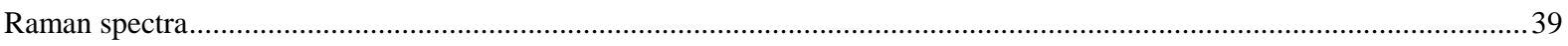

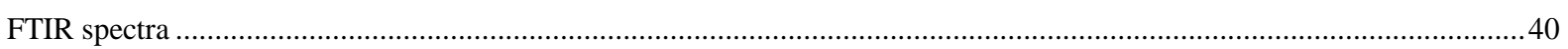

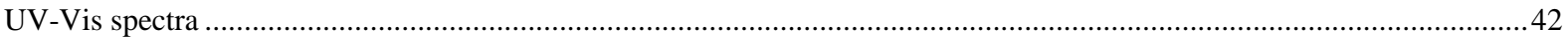

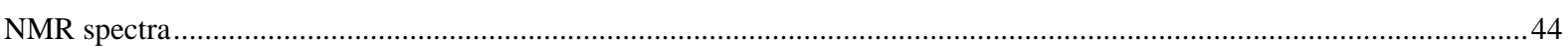

Table S4. Structurally characterized and reported transition metal dinitrogen dimers with different geometry on each metal

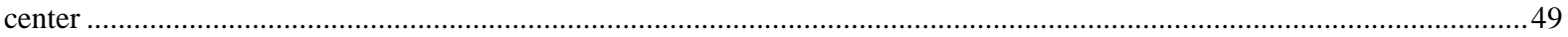

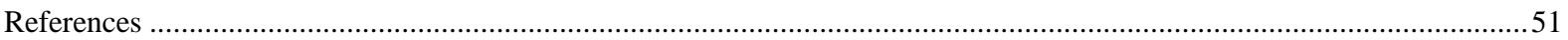




\section{General Considerations}

All air- and moisture-sensitive manipulations were performed using oven-dried or flame-dried glassware, including basic Schlenk and glovebox techniques under $\mathrm{N}_{2}$ atmosphere. Air- and moisture-insensitive reactions were carried out under an ambient atmosphere and magnetically stirred. Benzene, benzene- $d_{6}$, diethyl ether, toluene, toluene- $d_{8}$, and THF were distilled from deep purple sodium benzophenone ketyl. DMSO- $d_{6}$ was dried over activated $3 \AA$ molecular sieves and stored under $\mathrm{N}_{2}$. Ammonium chloride was sublimed for the calibration curve of the indophenol method. ${ }^{1}$ Unless stated otherwise, all other chemicals were used as received. Isotope enriched ${ }^{15} \mathrm{~N}_{2}$ was purchased from Aldrich. All deuterated solvents were purchased from Aldrich and Cambridge Isotope Laboratories. $\mathrm{PCy}_{3} \mathrm{HCl}_{2}{ }^{2} \mathrm{PCy}_{3} \mathrm{HI},{ }^{2} \mathrm{PCy}_{3} \mathrm{HBAr}_{4}{ }_{4}\left(\mathrm{BAr}_{4}{ }^{-}=\mathrm{B}\left\{3,5-\left(\mathrm{CF}_{3}\right)_{2} \mathrm{C}_{6} \mathrm{H}_{3}\right\} 4^{-}\right),{ }^{2} \mathrm{HBAr}_{4} \cdot 2 \mathrm{Et}_{2} \mathrm{O},{ }^{3}$ $(\mathbf{L 1}) \mathrm{Ti}^{\mathrm{IV}}(\mathrm{Cl})$ (complex 1; L1 = tris(4,6-di-tert-butyl-2-hydroxybenzyl)amine) ${ }^{4}, \mathbf{L} 2 \mathrm{H}_{3}(\mathbf{L 2}=\operatorname{tris}\{[2$-(hydroxy)2',4',6'-triisopropyl-3-biphenylyl]methyl $\}$ amine), ${ }^{5}$ and $\mathrm{KC}_{8}{ }^{6}$ were prepared following the literature procedures. NMR spectra were recorded on Bruker DRX 500/AVANCE III HD 850 spectrometers operating at 500/850 MHz $\left({ }^{1} \mathrm{H}\right), 125 / 212.5 \mathrm{MHz}\left({ }^{13} \mathrm{C}\right)$, and $50.7 / 86.2 \mathrm{MHz}\left({ }^{15} \mathrm{~N}\right) .{ }^{1} \mathrm{H}$ chemical shifts were referenced internally to the residual solvent peaks $\left(\mathrm{C}_{6} \mathrm{D}_{6}, \delta=7.16 / \mathrm{PhMe}-d_{8}, \delta=2.08,6.97,7.01,7.09 / \mathrm{DMSO}-d_{6}, \delta=2.50\right)$ of the deuterated solvent. ${ }^{13} \mathrm{C}$ chemical shifts were referenced to deuterated solvent peaks $\left(\mathrm{C}_{6} \mathrm{D}_{6}, \delta=128.06 / \mathrm{PhMe}-d_{8}, \delta=137.48,128.87\right.$, $127.96,125.13,20.43)$ of the deuterated solvent. ${ }^{15} \mathrm{~N}$ chemical shifts were referenced externally to $90 \%$ formamide in dimethyl sulfoxide- $d_{6}(\delta=112.4)$. Signals are listed in ppm, and multiplicity identified as $\mathrm{s}=$ singlet, $\mathrm{br}=\mathrm{broad}$, $\mathrm{d}=$ doublet, $\mathrm{t}=$ triplet, $\mathrm{q}=$ quartet, $\mathrm{m}=$ multiplet; coupling constants in $\mathrm{Hz}$; integration. Purified compounds were further dried under high vacuum $\left(\sim 10^{-3}\right.$ Torr). Solution state magnetic susceptibility was measured by Evans' $\operatorname{method}^{7}$ on a Bruker DRX 500 spectrometers in benzene- $d_{6}$ at 297 K. Raman spectra were recorded on a Renishaw inVia Raman microscope. Samples were measured as powders using a $633 \mathrm{~nm}$ laser at 50\% intensity. UV-VIS spectra were recorded using an Agilent Technologies Cary 6000i UV-Vis-NIR spectrometer. Samples were prepared under $\mathrm{N}_{2}$ in a sealed $1 \mathrm{~cm}$ path length cuvette. Infrared spectra were obtained as thin films formed by evaporation of solutions on $\mathrm{KBr}$ windows using a Bruker Alpha or Cary 600 system.

\section{Experimental Details}

Synthesis of ligand $\mathbf{L 1 H}_{3}$

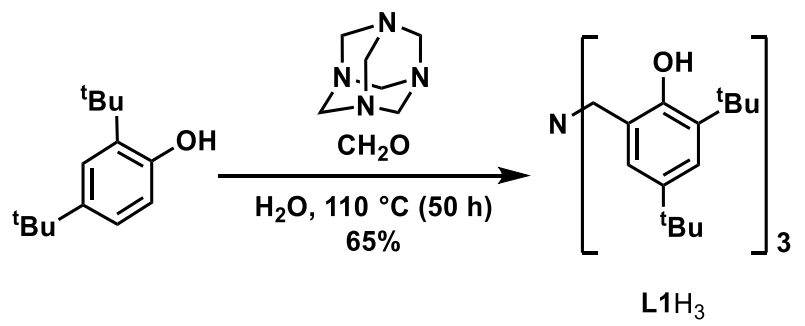

Tris(4,6-di-tert-butyl-2-hydroxybenzyl)amine was synthesized using a modified procedure from literature. ${ }^{8} 2,4-$ Di-tert-butylphenol (2.0 g, $9.7 \mathrm{mmol}, 12.0$ equiv.) and hexamethylenetetramine ( $0.114 \mathrm{~g}, 0.813 \mathrm{mmol}, 1.0$ equiv.) were added to a $20 \mathrm{ml}$ vial. Formalin $37 \% \mathrm{w} / \mathrm{w}(0.146 \mathrm{~g}, 4.9 \mathrm{mmol}, 6.0$ equiv. $)$ was added to the vial. The resulting mixture was stirred for 50 hours at $110{ }^{\circ} \mathrm{C}$. The reaction mixture was cooled to room temperature and $\mathrm{MeOH}$ was added to the vial. The vial was sonicated until yellow powder precipitated. The yellow powder was filtered and washed with $\mathrm{MeOH}(5 \times 3 \mathrm{~mL})$. The washed solid was dried to afford $\mathbf{L 1 H}_{3}$ as a colorless powder $(1.41 \mathrm{~g}, 65 \%)$.

${ }^{1} \mathrm{H}$ NMR $\left(500 \mathrm{MHz}, \mathrm{C}_{6} \mathrm{D}_{6}, 23{ }^{\circ} \mathrm{C}, \delta\right): 7.47$ (d, $\left.J=2.4 \mathrm{~Hz}, 3 \mathrm{H}\right), 6.99$ (d, J=2.4 Hz, 3H), 6.74 (br s, 3H), 3.40 (s, $6 \mathrm{H}), 1.57(\mathrm{~s}, 27 \mathrm{H}), 1.34(\mathrm{~s}, 27 \mathrm{H}) .{ }^{13} \mathrm{C} \mathrm{NMR}\left(125 \mathrm{MHz}, \mathrm{C}_{6} \mathrm{D}_{6}, 23{ }^{\circ} \mathrm{C}, \delta\right)=152.1,142.4,136.9,125.8,124.2,122.5$, $56.7,35.2,34.4,31.9,30.0$. 
Synthesis of $(\mathbf{L 1}) \mathrm{Ti}^{\mathrm{III}}\left(\mathrm{Et}_{2} \mathrm{O}\right)(\mathbf{2})$
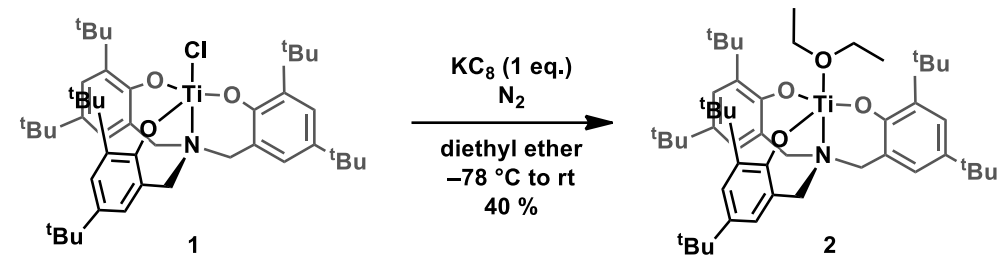

(L1) $\mathrm{Ti}^{\mathrm{IV}}(\mathrm{Cl})\left(50.0 \mathrm{mg}, 0.066 \mathrm{mmol}, 1.0\right.$ equiv.) and $\mathrm{KC}_{8}(9.0 \mathrm{mg}, 0.066 \mathrm{mmol}, 1.0$ equiv.) were added to a $20 \mathrm{~mL}$ vial followed by the addition of pre-cooled dry diethyl ether $\left(5 \mathrm{~mL},-78{ }^{\circ} \mathrm{C}\right)$. The mixture was left to warm to room temperature while stirring for 48 hours. The mixture was filtered through a pad of Celite, concentrated in vacuo, leading to precipitation of microcrystals, and left at room temperature for several days. The crystals were rinsed with a small amount of cold $\mathrm{Et}_{2} \mathrm{O}(3 \times 0.3 \mathrm{~mL})$ and dried in vacuo at $+23{ }^{\circ} \mathrm{C}$ to obtain 2 as light violet solid $(21.0 \mathrm{mg}, 40 \%)$. Despite multiple trials, we could not obtain satisfactory elemental analysis data due to high sensitivity of compound 2 . Solution magnetic moment $\left(\mathrm{C}_{6} \mathrm{D}_{6}, 300 \mathrm{~K}\right): 1.79 \mu_{\mathrm{B}}$.

Synthesis of $\left[\left\{(\mathbf{L} 1) \operatorname{Ti}^{\mathrm{IV}}{ }_{2}\left(\mu-\eta^{1}: \eta^{1}: \eta^{2}: \eta^{2}-\mathrm{N}_{2} \mathrm{~K}_{2}\right)(\mathrm{OEt}) \mathrm{K}\right](\mathbf{3})\right.$
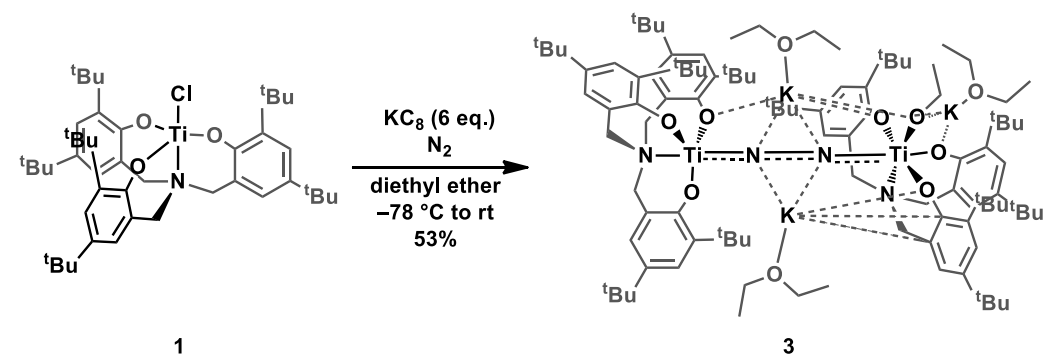

Method 1: $(\mathbf{L 1}) \mathrm{Ti}^{\mathrm{IV}}(\mathrm{Cl})\left(100.0 \mathrm{mg}, 0.133 \mathrm{mmol}, 1.0\right.$ equiv.) and $\mathrm{KC}_{8}(108.0 \mathrm{mg}, 0.798 \mathrm{mmol}, 6.0$ equiv. $)$ were added to a $20 \mathrm{~mL}$ vial followed by the addition of pre-cooled dry diethyl ether $\left(7 \mathrm{~mL},-78^{\circ} \mathrm{C}\right)$. The mixture was allowed to warm to room temperature while stirring over 48 hours. The mixture was filtered through a pad of Celite, concentrated in vacuo, leading to precipitation of microcrystals, and left at room temperature for several days. The crystals were rinsed with a small amount of cold $\mathrm{Et}_{2} \mathrm{O}(3 \times 0.3 \mathrm{~mL})$ and dried in vacuo at $+23{ }^{\circ} \mathrm{C}$ to afford 2 as a brownish purple solid $(62.2 \mathrm{mg}, 53 \%) .{ }^{1} \mathrm{H}$ NMR $\left(500 \mathrm{MHz}, \mathrm{PhMe}-d_{8}, 23{ }^{\circ} \mathrm{C}, \delta\right): 7.44(\mathrm{~d}, J=2.27$ $\mathrm{Hz}, 3 \mathrm{H}), 7.33(\mathrm{~d}, J=2.36 \mathrm{~Hz}, 1 \mathrm{H}), 7.19-7.16(\mathrm{~m}, 4 \mathrm{H}), 6.85(\mathrm{~d}, J=2.43 \mathrm{~Hz}, 3 \mathrm{H}), 6.34-6.33(\mathrm{~m}, 1 \mathrm{H}), 5.24(\mathrm{~d}, J=$ $13.6 \mathrm{~Hz}, 1 \mathrm{H}), 5.03(\mathrm{~d}, J=14.1 \mathrm{~Hz}, 1 \mathrm{H}), 4.66(\mathrm{q}, J=7.5 \mathrm{~Hz}, 1 \mathrm{H}), 4.43(\mathrm{q}, J=7.0 \mathrm{~Hz}, 1 \mathrm{H}), 3.93-3.90(\mathrm{~m}, 1 \mathrm{H})$, $3.72(\mathrm{~d}, J=12.6 \mathrm{~Hz}, 3 \mathrm{H}), 3.52(\mathrm{~m}, 1 \mathrm{H}), 3.41(\mathrm{~d}, J=14.5 \mathrm{~Hz}, 1 \mathrm{H}), 2.78(\mathrm{~d}, J=12.6 \mathrm{~Hz}, 1 \mathrm{H}), 2.47(\mathrm{~d}, J=13.1$ $\mathrm{Hz}, 3 \mathrm{H}), 1.67-1.66(\mathrm{~m}, 3 \mathrm{H}), 1.54(\mathrm{~s}, 9 \mathrm{H}), 1.53(\mathrm{~s}, 27 \mathrm{H}), 1.38-1.37$ (m, 9H), $1.36(\mathrm{~s}, 27 \mathrm{H}), 1.34-1.33$ (m, 9H), 1.29 (s, 9H), 1.25 (s, 9H), 1.24 (s, 9H). ${ }^{13} \mathrm{C}$ NMR $\left(125 \mathrm{MHz}, \mathrm{PhMe}-d_{8}, 23{ }^{\circ} \mathrm{C}, \delta\right): 161.6,138.3,135.2,135.0$, $134.8,134.3,132.2,126.6,126.2,124.7,124.4,123.1,123.0,122.9,121.9,71.9,67.8,65.9,58.6,40.5,35.3,34.3$, $34.2,33.8,32.9,32.5,32.4,32.4,32.1,31.9,31.2,30.8,30.7,30.6,30.6,30.2,30.2,30.1,15.6,15.2$. (Raman (cm $\left.{ }^{1}\right): 1304\left(\left({ }^{14} \mathrm{~N}-{ }^{14} \mathrm{~N}\right)\right)$. 


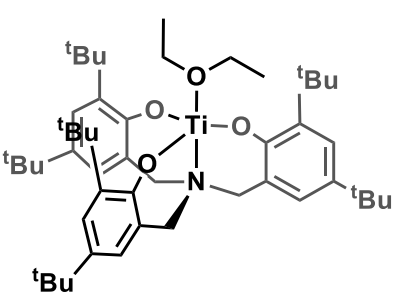

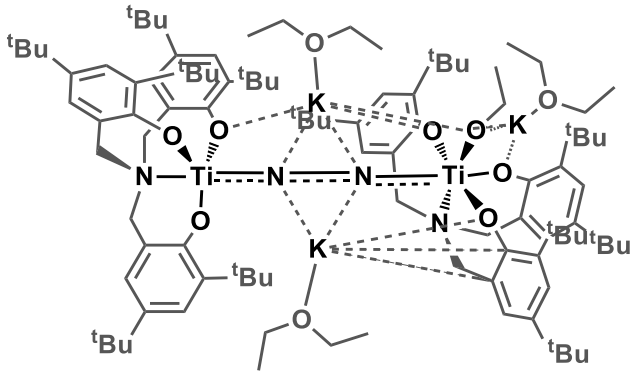

3

Method 2: 2 (30.0 mg, $0.038 \mathrm{mmol}, 1.0$ equiv.) and $\mathrm{KC}_{8}(20.5 \mathrm{mg}, 0.152 \mathrm{~mol}, 4.0$ equiv.) were added to a $20 \mathrm{~mL}$ vial followed by the addition of pre-cooled dry diethyl ether $\left(3 \mathrm{~mL},-78{ }^{\circ} \mathrm{C}\right)$. The mixture was allowed to warm to room temperature while stirring over 48 hours. The mixture was filtered through a pad of Celite, concentrated in vacuo, leading to precipitation of microcrystals, and left at room temperature for several days. The crystals were rinsed with a small amount of cold $\mathrm{Et}_{2} \mathrm{O}(3 \times 0.3 \mathrm{~mL})$ and dried in vacuo at $+23{ }^{\circ} \mathrm{C}$ to afford 2 as a brownishpurple solid (15.9 mg, 45\%).

Synthesis of $\left[\left\{(\mathbf{L 1}) \mathrm{T}_{2}\left(\mu-\eta^{1}: \eta^{1}: \eta^{2}: \eta^{2}-{ }^{15} \mathrm{~N}_{2} \mathrm{~K}_{2}\right)(\mathrm{OEt}) \mathrm{K}\right]\left(\mathbf{3}^{\mathbf{1 5}} \mathbf{N}_{2}\right)\right.$

(L1) $\mathrm{Ti}^{\mathrm{IV}}(\mathrm{Cl})\left(100 \mathrm{mg}, 0.133 \mathrm{mmol}, 1.0\right.$ equiv.) and $\mathrm{KC}_{8}(108 \mathrm{mg}, 0.798 \mathrm{mmol}, 6.0$ equiv.) were added to bulb $\mathrm{A}$ of the H-shape apparatus (Figure $\mathrm{S} 10)^{2,9}$ and $7 \mathrm{~mL}$ of diethyl ether to bulb $\mathrm{B}$ of the apparatus. Each bulb was freeze-pump-thaw degassed three times independently then exposed to ${ }^{15} \mathrm{~N}_{2}$ at room temperature. The solvent was transferred to bulb A and the reaction mixture was stirred for 48 hours, slowly warming up from $-78^{\circ} \mathrm{C}$ to room temperature. The mixture was filtered through a pad of Celite, concentrated in vacuo, leading to precipitation of microcrystals, and left at room temperature for several days. The crystals were rinsed with a small amount of cold $\mathrm{Et}_{2} \mathrm{O}(3 \times 0.3 \mathrm{~mL})$ and dried in vacuo at $+23{ }^{\circ} \mathrm{C}$ to afford $3-{ }^{15} \mathbf{N}_{2}$ as a brown solid $(18 \mathrm{mg}, 8 \%) .{ }^{15} \mathrm{~N} \mathrm{NMR}(\mathrm{PhMe}-$ $\left.d_{8}, 23{ }^{\circ} \mathrm{C}\right): \delta(\mathrm{ppm}) 411.08(\mathrm{dd}, J=72.2,13.6 \mathrm{~Hz}), 392.97(\mathrm{dd}, J=47.6,13.6 \mathrm{~Hz})$. Raman $\left(\mathrm{cm}^{-1}\right): 1250\left(\left({ }^{15} \mathrm{~N}-\right.\right.$ $\left.{ }^{15} \mathrm{~N}\right)$ ). All other spectroscopic data were consistent with 3.

Synthesis of $(\mathbf{L} 2) \mathrm{Ti}^{\mathrm{IV}}(\mathrm{Cl})(\mathbf{4})$

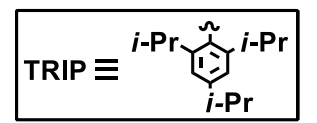<smiles>CCc1cccc([In]2CCCC2)c1O</smiles>

$\mathrm{L}^{2} \mathrm{H}_{3}$

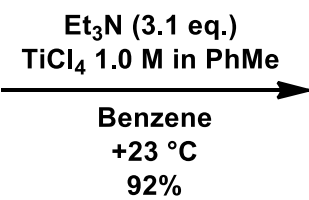

$92 \%$

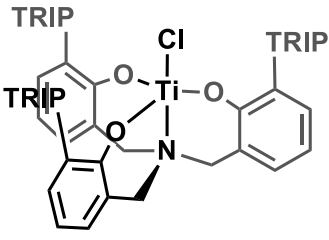

4

$\mathbf{2} 2 \mathrm{H}_{3}$ (150.0 mg, $0.159 \mathrm{mmol}, 1.0$ equiv.) was added to a $20 \mathrm{~mL}$ vial followed by addition of $7 \mathrm{~mL}$ of dry benzene and triethylamine $(68.8 \mu \mathrm{L}, 0.493 \mathrm{mmol}, 3.1$ equiv. $) .1 .0 \mathrm{M}$ of $\mathrm{TiCl}_{4}(159 \mathrm{~mL}, 0.159 \mu \mathrm{mol}, 1.0$ equiv. $)$ in $\mathrm{PhMe}$ was added dropwise to the vial. The mixture was stirred at $+23{ }^{\circ} \mathrm{C}$ for 12 hours. The solution was filtered through a pad of Celite and volatiles were removed in vacuo to obtain $\mathbf{4}$ as a colorless solid (149.3 mg, 92\%). X-ray quality single crystals were grown by vapor diffusion $\left(\mathrm{C}_{6} \mathrm{H}_{6} /\right.$ pentane) at room temperature over 14 days. ${ }^{1} \mathrm{H}$ NMR $(500$ $\left.\mathrm{MHz}, \mathrm{C}_{6} \mathrm{D}_{6}, 23^{\circ} \mathrm{C}, \delta\right): 7.19(\mathrm{~d}, J=1.50 \mathrm{~Hz}, 3 \mathrm{H}), 7.15(\mathrm{~d}, J=1.50 \mathrm{~Hz}, 3 \mathrm{H}) 7.11-7.09(\mathrm{~m}, 3 \mathrm{H}), 6.87-6.86(\mathrm{~m}, 6 \mathrm{H})$, $4.14(\mathrm{~d}, J=14.3 \mathrm{~Hz}, 3 \mathrm{H}), 2.82$ (sept, $J=6.8 \mathrm{~Hz}, 3 \mathrm{H}), 2.78$ (sept, $J=6.8 \mathrm{~Hz}, 3 \mathrm{H}), 2.62(\mathrm{~d}, J=14.3 \mathrm{~Hz}, 3 \mathrm{H}), 2.50$ $(\mathrm{sept}, J=6.8 \mathrm{~Hz}, 3 \mathrm{H}), 1.41(\mathrm{~d}, J=6.9 \mathrm{~Hz}, 9 \mathrm{H}), 1.25(\mathrm{~d}, J=6.9 \mathrm{~Hz}, 9 \mathrm{H}), 1.23(\mathrm{~d}, J=6.9 \mathrm{~Hz}, 9 \mathrm{H}), 1.21(\mathrm{~d}, J=6.9$ $\mathrm{Hz}, 9 \mathrm{H}), 1.12$ (d, $J=6.9 \mathrm{~Hz}, 9 \mathrm{H}), 0.99$ (d, $J=6.9 \mathrm{~Hz}, 9 \mathrm{H}) .13 \mathrm{C}$ NMR $\left(125 \mathrm{MHz}, \mathrm{C}_{6} \mathrm{D}_{6}, 23{ }^{\circ} \mathrm{C}, \delta\right): 161.2,148.9$, 
$147.4,146.3,131.8,131.7,130.2,129.1,123.9,122.0,121.2,120.3,57.7,34.9,31.4,31.1,25.5,24.6,24.5,24.2$, 23.9. Anal. Calcd. for $\mathrm{C}_{66} \mathrm{H}_{84} \mathrm{ClNO}_{3}$ Ti: $\mathrm{C}, 77.51 ; \mathrm{H}, 8.28 ; \mathrm{N}, 1.37$; found: $\mathrm{C}, 77.59 ; \mathrm{H}, 8.66 ; \mathrm{N}, 1.84$.

Synthesis of (L2) $\mathrm{Ti}^{\mathrm{III}}(\mathrm{KOEt})(\mathbf{5})$

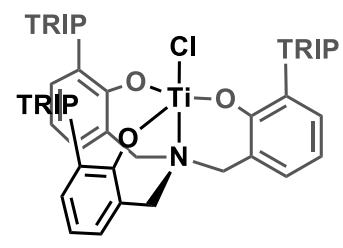

4

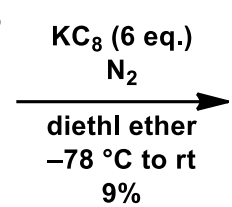

9\%

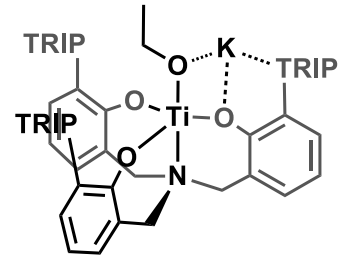

5

4 (40.0 mg, $0.039 \mathrm{mmol}, 1.0$ equiv.) and $\mathrm{KC}_{8}(31.7 \mathrm{mg}, 0.235 \mathrm{mmol}, 6.0$ equiv.) was added to a $20 \mathrm{~mL}$ vial followed by the addition of pre-cooled dry diethyl ether $\left(3 \mathrm{~mL},-78^{\circ} \mathrm{C}\right)$. The mixture was allowed to warm to room temperature while stirring over 48 hours. The volatiles were removed in vacuo. The solid residue was extracted with pentane $(3 \times 0.3 \mathrm{~mL})$. The mixture was filtered through a pad of Celite, concentrated in vacuo, and left at $-40{ }^{\circ} \mathrm{C}$ freezer for 5 months. The crystals were rinsed with a small amount of cold pentane $(3 \times 0.1 \mathrm{~mL})$ and dried in vacuo at $+23{ }^{\circ} \mathrm{C}$ to afford 5 as brownish-violet solid (3.6 mg, $\left.9 \%\right)$. Solution magnetic moment $\left(\mathrm{C}_{6} \mathrm{D}_{6}\right.$, $300 \mathrm{~K}): 1.86 \mu_{\mathrm{B}}$.

Ethylene detection in the reduction of $\mathbf{1}$ to $\mathbf{3}$

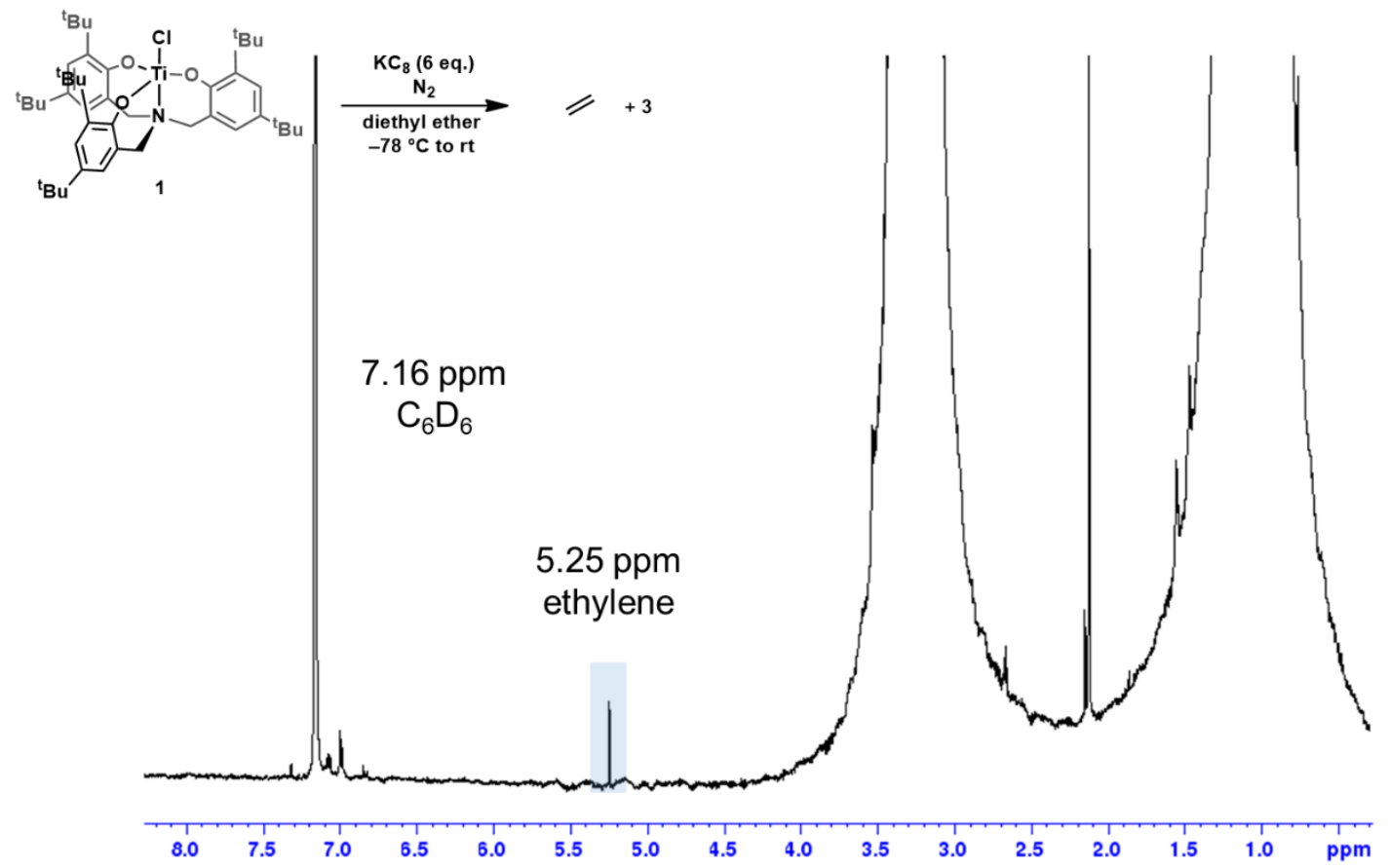

Figure S1. Vacuum transferred ${ }^{1} \mathrm{H}$ NMR spectrum of the reaction mixture of 1 and 6 equivalents of $\mathrm{KC}_{8}$

Complex 1 (30.0 mg, $40.0 \mu \mathrm{mol}, 1.0$ equiv.) and $\mathrm{KC}_{8}(32.3 \mathrm{mg}, 240.0 \mu \mathrm{mol}, 6.0$ equiv.) was added to a $25 \mathrm{~mL}$ Schlenk flask, followed by the addition of pre-cooled dry diethyl ether $\left(1.5 \mathrm{~mL},-78^{\circ} \mathrm{C}\right)$. The mixture was left to warm to room temperature while stirring for 48 hours. $\mathrm{C}_{6} \mathrm{D}_{6}(600 \mu \mathrm{L})$ which freeze-pump-thaw cycle proceeded three times in a JY NMR tube was prepared. The reaction mixture was vacuum transferred to the JY tube. Ethylene was detected by ${ }^{1} \mathrm{H}$ NMR. ${ }^{1} \mathrm{H}$ NMR $\left(500 \mathrm{MHz}, \mathrm{C}_{6} \mathrm{D}_{6}, 23{ }^{\circ} \mathrm{C}, \delta\right): 5.25$ (s, 4H). 
Reaction of the ethoxide ligand in $\mathbf{3}$ with TBDMS-Cl

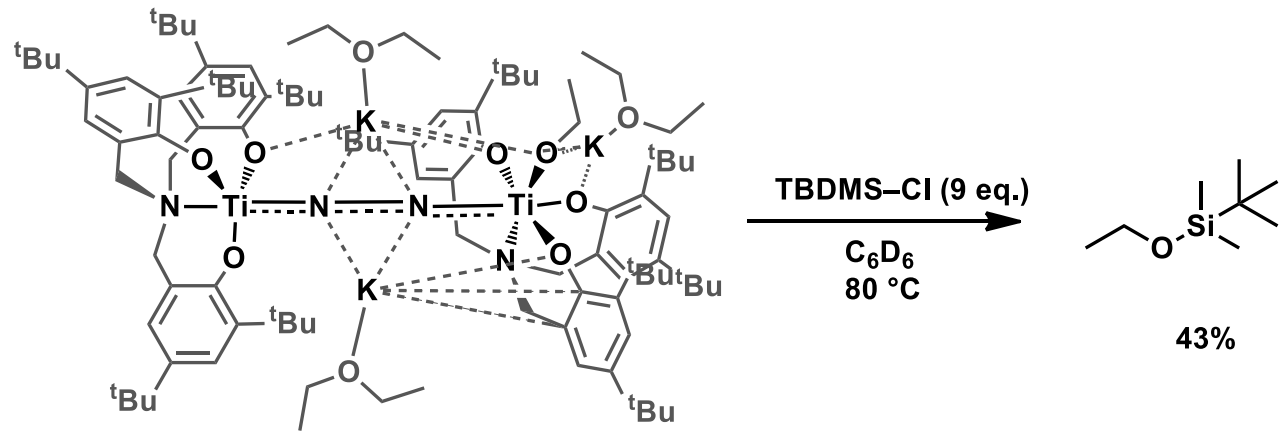

Complex 3 (5.0 mg, $2.71 \mu \mathrm{mol}, 1.0$ equiv.) was added to a JY NMR tube, followed by $600 \mu \mathrm{L}_{\text {of }} \mathrm{C}_{6} \mathrm{D}_{6}$ and tertbutyldimethylsilyl chloride $\left(3.8 \mathrm{mg}, 25.0 \mu \mathrm{mol}, 9.0\right.$ equiv.). The reaction mixture was heated at $80^{\circ} \mathrm{C}$ for 17 hours. After cooling down to room temperature, the volatiles were vacuum transferred to another JY tube and analyzed by ${ }^{1} \mathrm{H}$ NMR spectroscopy and GC-MS (Figure S2 and S3). The ${ }^{1} \mathrm{H}$ NMR yield of dimethyl-tert-butylethoxysilane was $43 \%$ using dibromomethane as an internal standard (Figure S2). Some chemical shifts were overlaid by volatiles including $\mathrm{Et}_{2} \mathrm{O}$, therefore, to analyze precisely, dimethyl-tert-butylethoxysilane was prepared independently by salt metathesis of TBDMS-Cl $(6.3 \mathrm{mg}, 42 \mu \mathrm{mol}, 1.0$ equiv.) and KOEt (3.5 mg, $42 \mu \mathrm{mol}, 1.0$ equiv.) in $\mathrm{C}_{6} \mathrm{D}_{6}$ for 17 hours at $80{ }^{\circ} \mathrm{C}$. The product was characterized by ${ }^{1} \mathrm{H}$ NMR and GC-MS, which agrees with the results described in Figures S4-S6. ${ }^{1} \mathrm{H}$ NMR $\left(500 \mathrm{MHz}, \mathrm{C}_{6} \mathrm{D}_{6}, 23{ }^{\circ} \mathrm{C}, \delta\right): 3.54(\mathrm{q}, J=6.9 \mathrm{~Hz}, 2 \mathrm{H}), 1.10(\mathrm{t}, J=$ $7.0 \mathrm{~Hz}, 3 \mathrm{H}), 0.98$ (s, 9H), 0.05 (s, 6H). GC-MS: 160.1 Da $\left(\mathrm{M}^{+}\right)$.

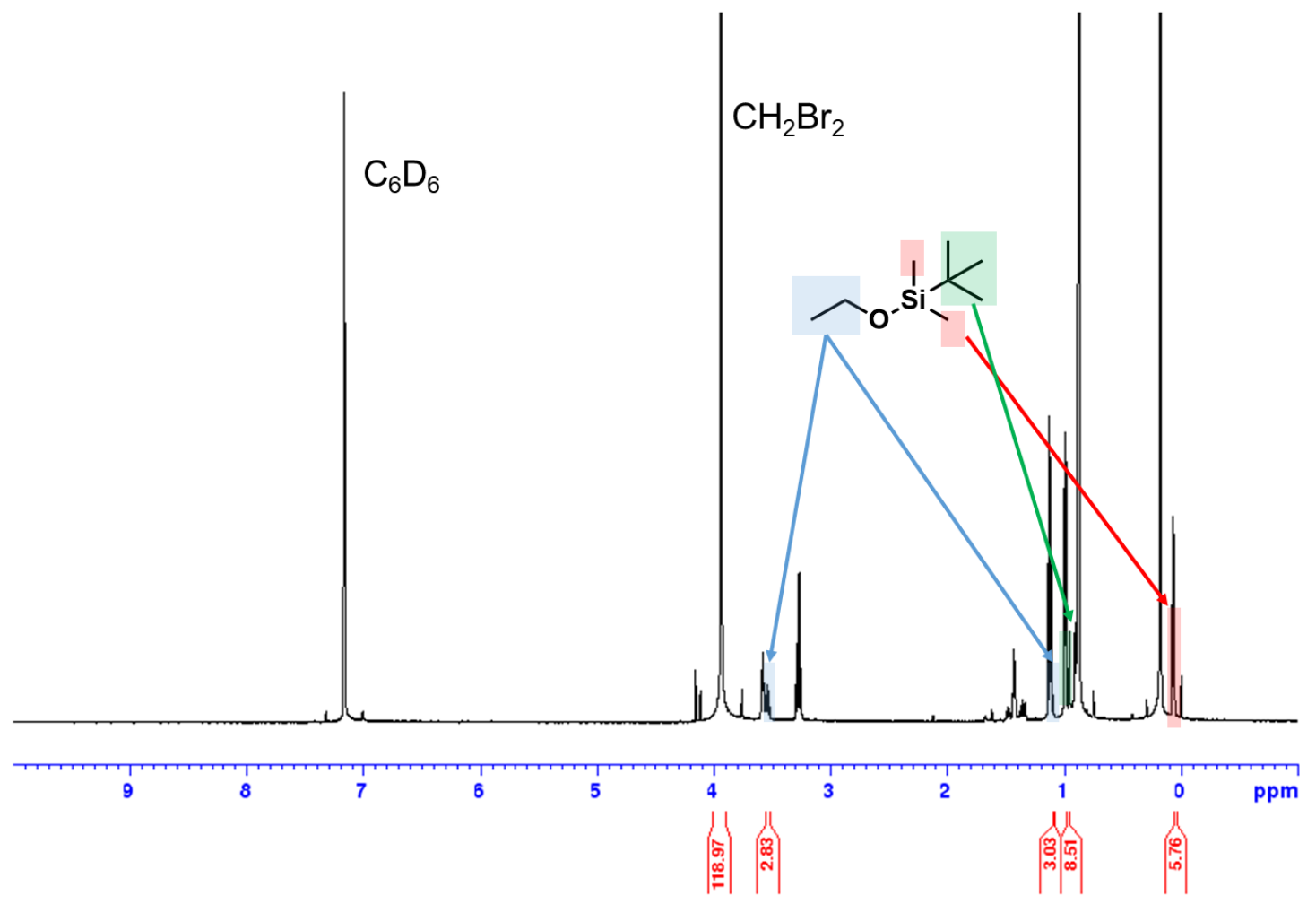

Figure S2. ${ }^{1} \mathrm{H}$ NMR spectrum of the vacuum transferred reaction mixture of 3 and TBDMS-Cl in $\mathrm{C}_{6} \mathrm{D}_{6}$. 
Abundance

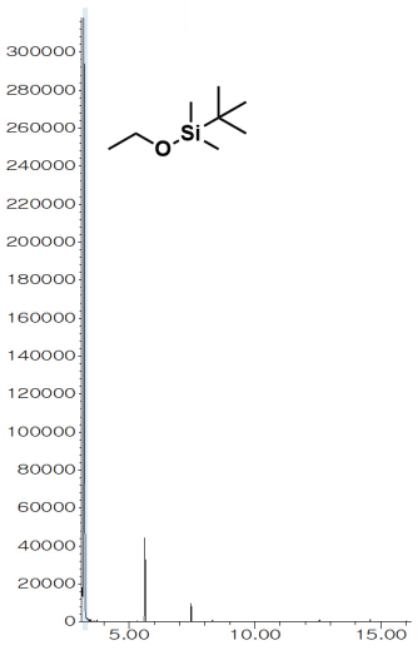

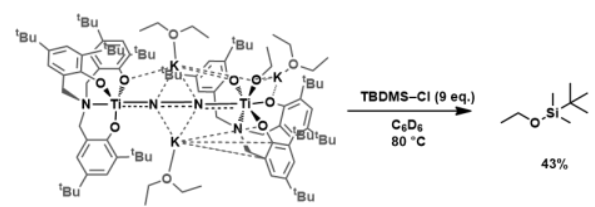

Abundance

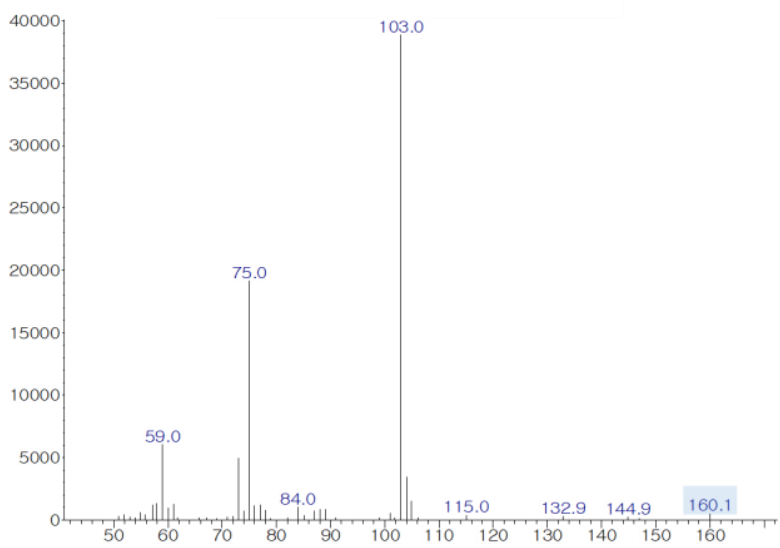

Figure S3. GC-MS spectrum (left) and observed MS spectrum (right) of the vacuum transferred reaction mixture of 3 and TBDMS- $\mathrm{Cl}$ in $\mathrm{C}_{6} \mathrm{D}_{6}$.

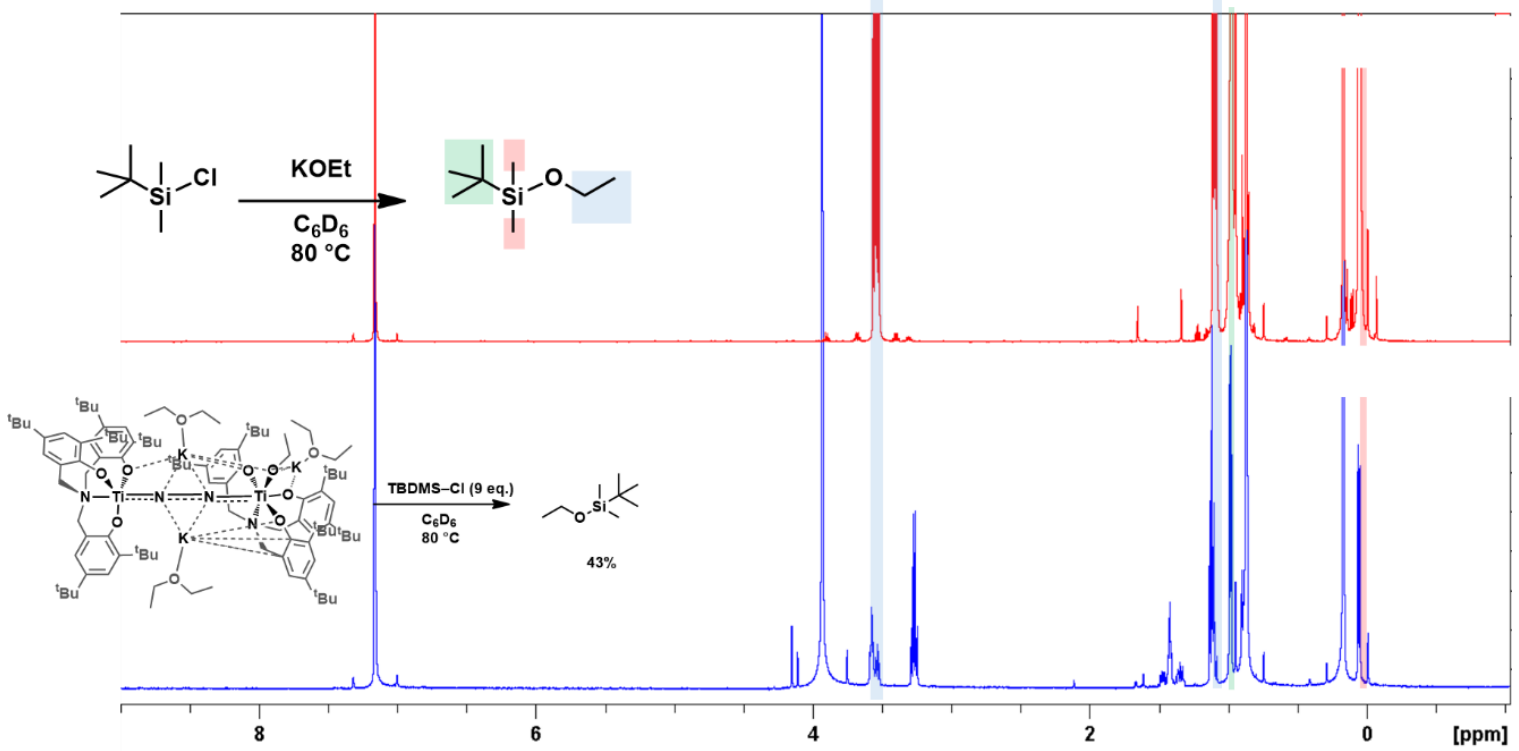

Figure S4. Comparison of ${ }^{1} \mathrm{H}$ NMR spectra of the reaction of TBDMS- $\mathrm{Cl}$ and KOEt and $\mathbf{3}$ and TBDMS-Cl in $\mathrm{C}_{6} \mathrm{D}_{6}$. 


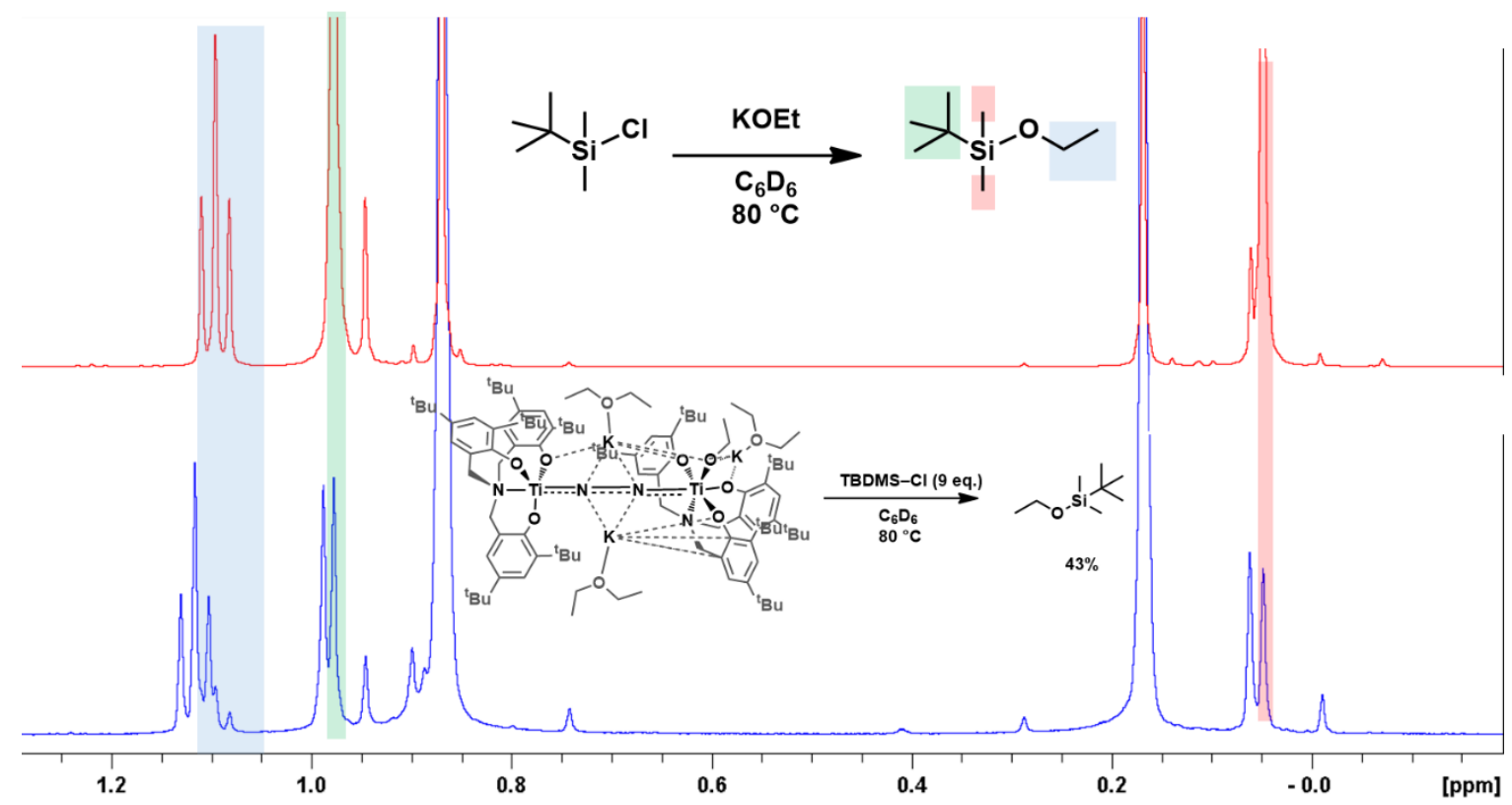

Figure S5. Comparison of ${ }^{1} \mathrm{H}$ NMR spectra of the reaction of TBDMS- $\mathrm{Cl}$ and KOEt and $\mathbf{3}$ and TBDMS-Cl in the upfield region $\left(\mathrm{C}_{6} \mathrm{D}_{6}\right)$.

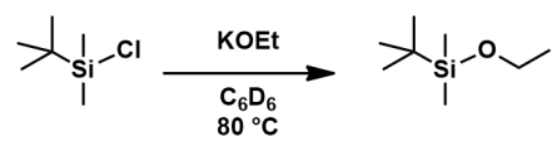

Abundance

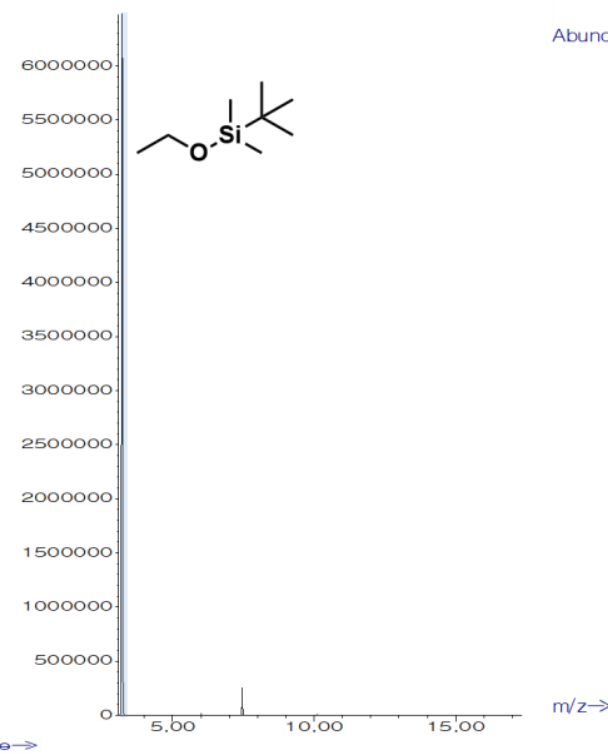

Abundance

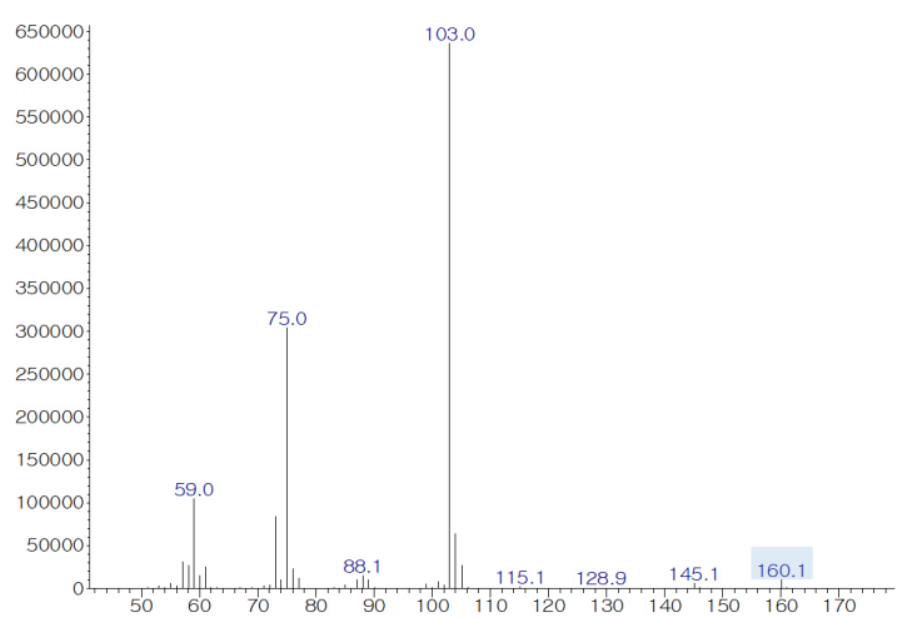

Figure S6. GC-MS spectrum (left) and observed MS spectrum (right) of the reaction mixture of TBDMS-Cl, and KOEt in $\mathrm{C}_{6} \mathrm{D}_{6}$. 
Spectroscopic monitoring of the reaction of 1 with 3 equivalents of $\mathrm{KC}_{8}$
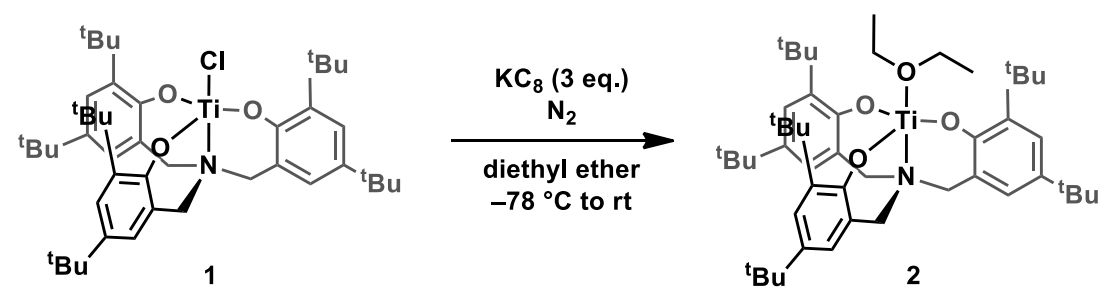

1 (10.0 mg, $13.3 \mu \mathrm{mol}, 1.0$ equiv.) and $\mathrm{KC}_{8}(5.4 \mathrm{mg}, 39.9 \mu \mathrm{mol}, 3.0$ equiv.) were added to a $4 \mathrm{~mL}$ vial followed by the addition of pre-cooled dry diethyl ether $\left(1 \mathrm{~mL},-78{ }^{\circ} \mathrm{C}\right)$. The mixture was allowed to warm to room temperature while stirring for 48 hours. The mixture was filtered through a pad of Celite and analyzed by IR spectroscopy. The residual solution was evaporated to dryness and analyzed by ${ }^{1} \mathrm{H}$ NMR spectroscopy in PhMe$d_{8}$.
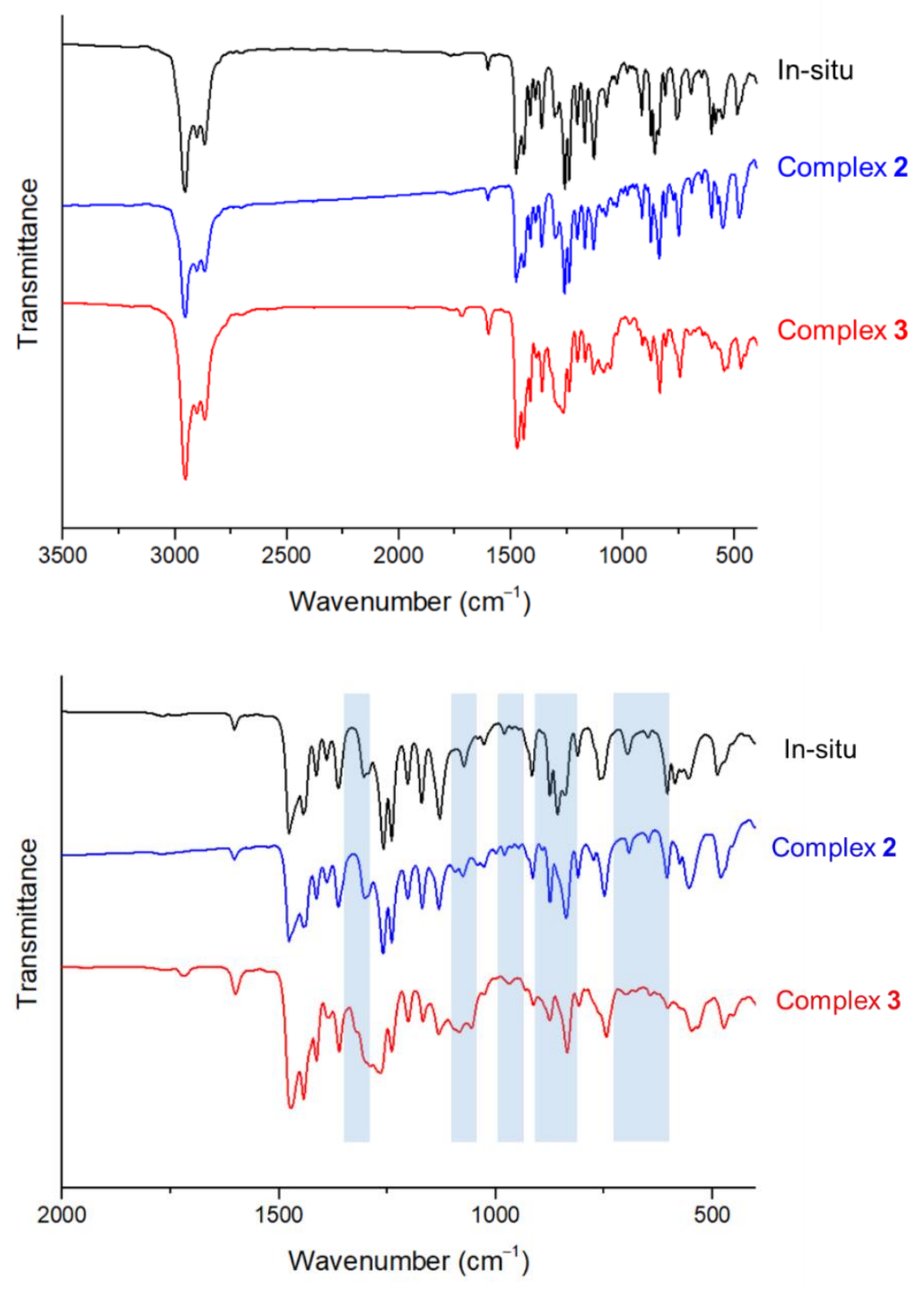

Figure S7. Comparison of IR spectra, black: in-situ reaction mixture from the reduction of $\mathbf{1}$ using $\mathrm{KC}_{8}(3 \mathrm{eq}$.) blue: complex 2 red: complex 3 (top: whole range, bottom: IR range from $2000 \mathrm{~cm}^{-1}$ to $400 \mathrm{~cm}^{-1}$ ). 


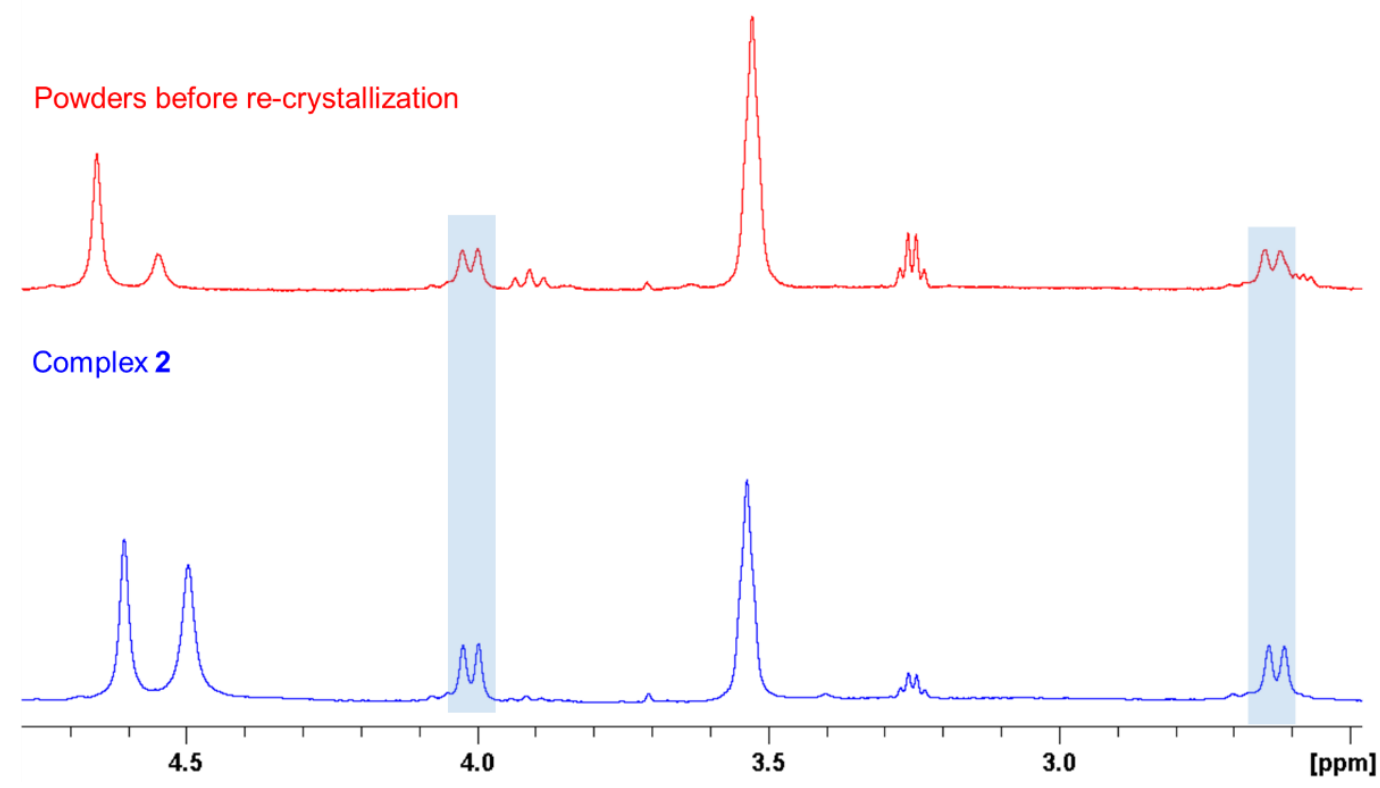

Figure S8. Comparison of ${ }^{1} \mathrm{H}$ NMR spectra in $\mathrm{PhMe}-d_{8}$, red: in-situ reaction mixture from the reduction of 1 using $\mathrm{KC}_{8}$ (3 eq.) blue: complex 2.

Comparison of UV-VIS spectra of 2 at $293 \mathrm{~K}$ and $233 \mathrm{~K}$.

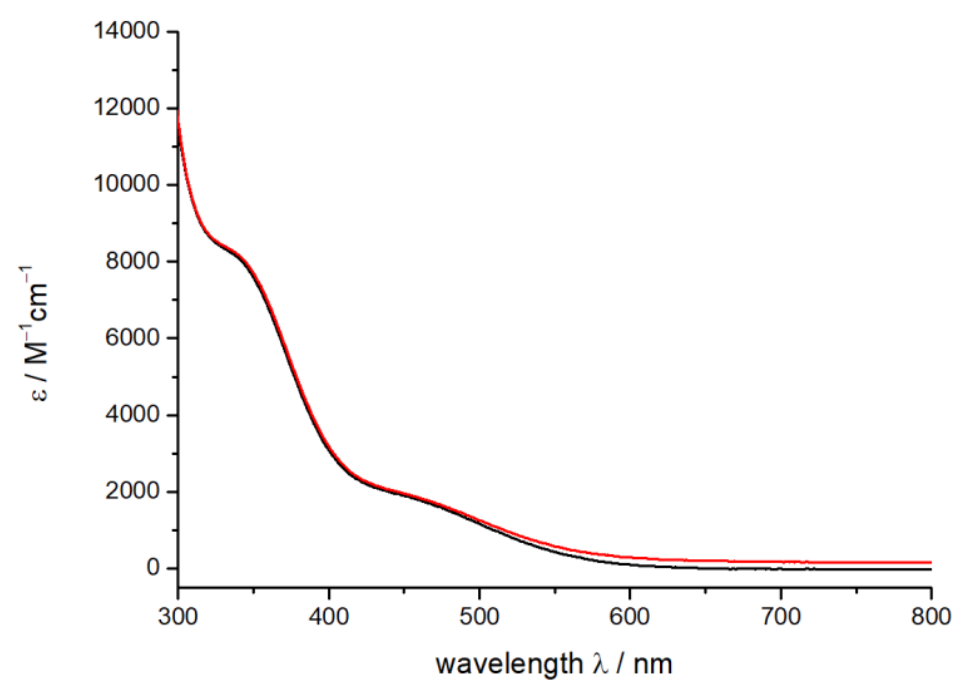

Figure S9. Comparison of UV-VIS spectra of 2 at $293 \mathrm{~K}$ (black trace) and $233 \mathrm{~K}$ (red trace) in $0.1 \mathrm{mM}$ PhMe. No change was found when 2 was stored at lower temperature $\left(-40^{\circ} \mathrm{C}\right)$ for 24 hours. 
In a nitrogen atmosphere glovebox, the complex $3(4.0 \mathrm{mg}, 2.17 \mu \mathrm{mol})$ was weighed into a $50 \mathrm{~mL}$ Schlenk flask equipped with a magnetic stirring bar. Then, 150 equivalents of a proton source and 150 equivalents of an electron source were added to the flask. The flask is cooled to $77 \mathrm{~K}$ in a cold well using liquid nitrogen. A pre-cooled solvent $(4 \mathrm{~mL})$ was added to the flask which was sealed subsequently. The reaction mixture was kept in the cold well for 5 minutes before transferring to a dry ice/acetone bath outside of the glovebox. Then, it was stirred in the cold bath for 10 hours and allowed to warm to room temperature for further 19 hours.

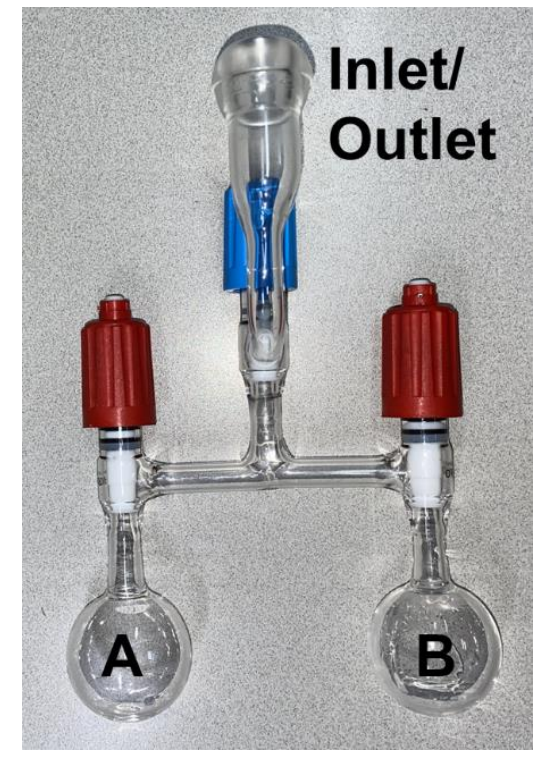

Figure S10. H-shape apparatus for ${ }^{15} \mathrm{~N}_{2}$ experiments

Standard acidification procedure under ${ }^{15} \mathrm{~N}_{2}$ atmosphere

In a nitrogen atmosphere glovebox, the complex $3-{ }^{15} \mathbf{N}_{2}(4.0 \mathrm{mg}, 2.17 \mu \mathrm{mol})$ was weighed into an H-shape apparatus (Figure S10). Then, 150 equivalents of a proton source and 150 equivalents of an electron source were added to bulb A of the apparatus, containing a magnetic stirring bar, and $4 \mathrm{~mL}$ of a solvent was added to bulb $\mathrm{B}$. The sealed apparatus was connected to a Schlenk line and freeze-pump-thaw degassing was performed 5 times on bulbs A and B. The apparatus was filled with ${ }^{15} \mathrm{~N}_{2}$ gas, bulb A was cooled in a liquid nitrogen bath and the solvent was transferred from bulb B to bulb A. The mixture was stirred at $-78{ }^{\circ} \mathrm{C}$ for 10 hours and allowed to warm to room temperature for further 19 hours.

\section{Ammonia and hydrazine quantification}

A Schlenk flask was filled with $3 \mathrm{~mL}$ of $2.0 \mathrm{M} \mathrm{HCl}$ in diethyl ether to trap $\mathrm{NH}_{3}$. A glass vacuum transfer apparatus was attached to a high-vacuum $\left(<10^{-3}\right.$ Torr) line and evacuated for $15 \mathrm{~min}$. A reaction vessel and the trap $(3 \mathrm{~mL}$ of $2.0 \mathrm{M} \mathrm{HCl}$ in diethyl ether) were connected to the apparatus, and then both of them were cooled with liquid nitrogen for 15 minutes. The reaction mixture was thawed and the remaining volatiles were vacuum-transferred to the trap under static vacuum. After the vacuum-transfer, the reaction vessel was taken into an $\mathrm{N}_{2}$ filled glovebox. To the solid residue in the reaction vessel, $40 \mathrm{mg}$ of potassium tert-butoxide in MeOH/THF (4 mL/ $2 \mathrm{~mL}) \mathrm{was}$ added. The flask was taken out of the glovebox and attached to the vacuum transfer apparatus. The homogeneous solution was stirred for 15 minutes and the volatiles were vacuum-transferred to the trap. All volatiles in the trap were removed under dynamic vacuum at room temperature. (I) The remaining solid including ammonium chloride in the trap was dissolved in $1 \mathrm{~mL}$ of $\mathrm{H}_{2} \mathrm{O}$. The amount of ammonia was quantified by the indophenol method ${ }^{1}$ 
using an aliquot of the solution $(20 \mu \mathrm{L})$. The indophenol absorbance was calibrated using sublimed $\mathrm{NH}_{4} \mathrm{Cl}$ within the range of 0.035 to 2.49 , and the absorbance data of all samples were interpolated in that region. (II) Otherwise, the quantification of ammonia was also conducted by integrating ${ }^{1} \mathrm{H}-\mathrm{NMR}$ peaks. The solid residue in the trap was extracted into $600 \mu \mathrm{L}$ of DMSO- $d_{6}$ solvent containing 1,3,5-trimethoxybenzene as an internal standard. Ammonium peaks in ${ }^{1} \mathrm{H}$-NMR were integrated against the two peaks of the internal standard. The amount of $\mathrm{N}_{2} \mathrm{H}_{4}$ was determined by the $p$-(dimethylamino)benzaldehyde method. ${ }^{10}$

Calibration curve for ammonia quantification

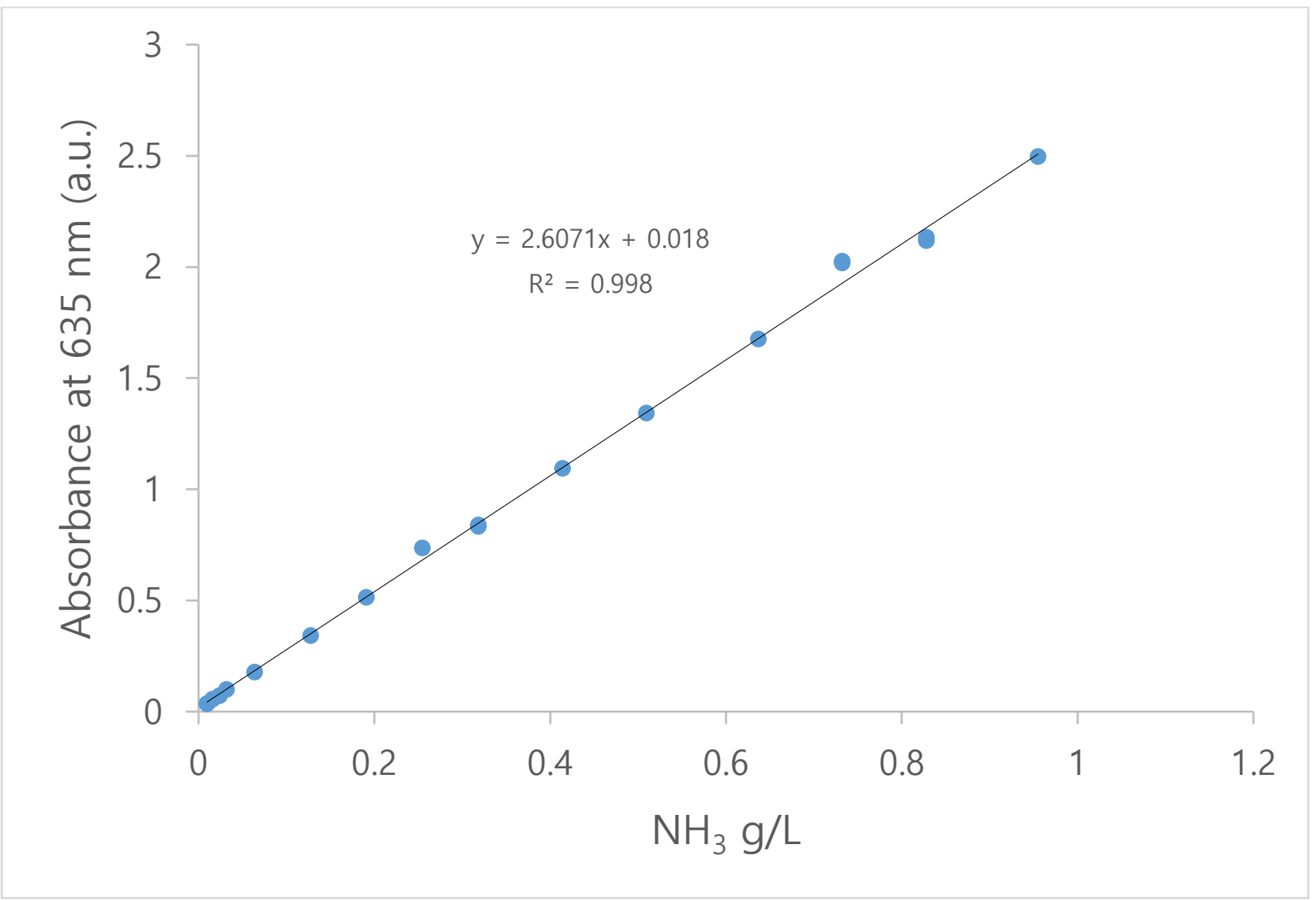

Figure S11. Calibration curve for $\mathrm{NH}_{3}$ UV-VIS quantification 
Calibration curve for hydrazine quantification

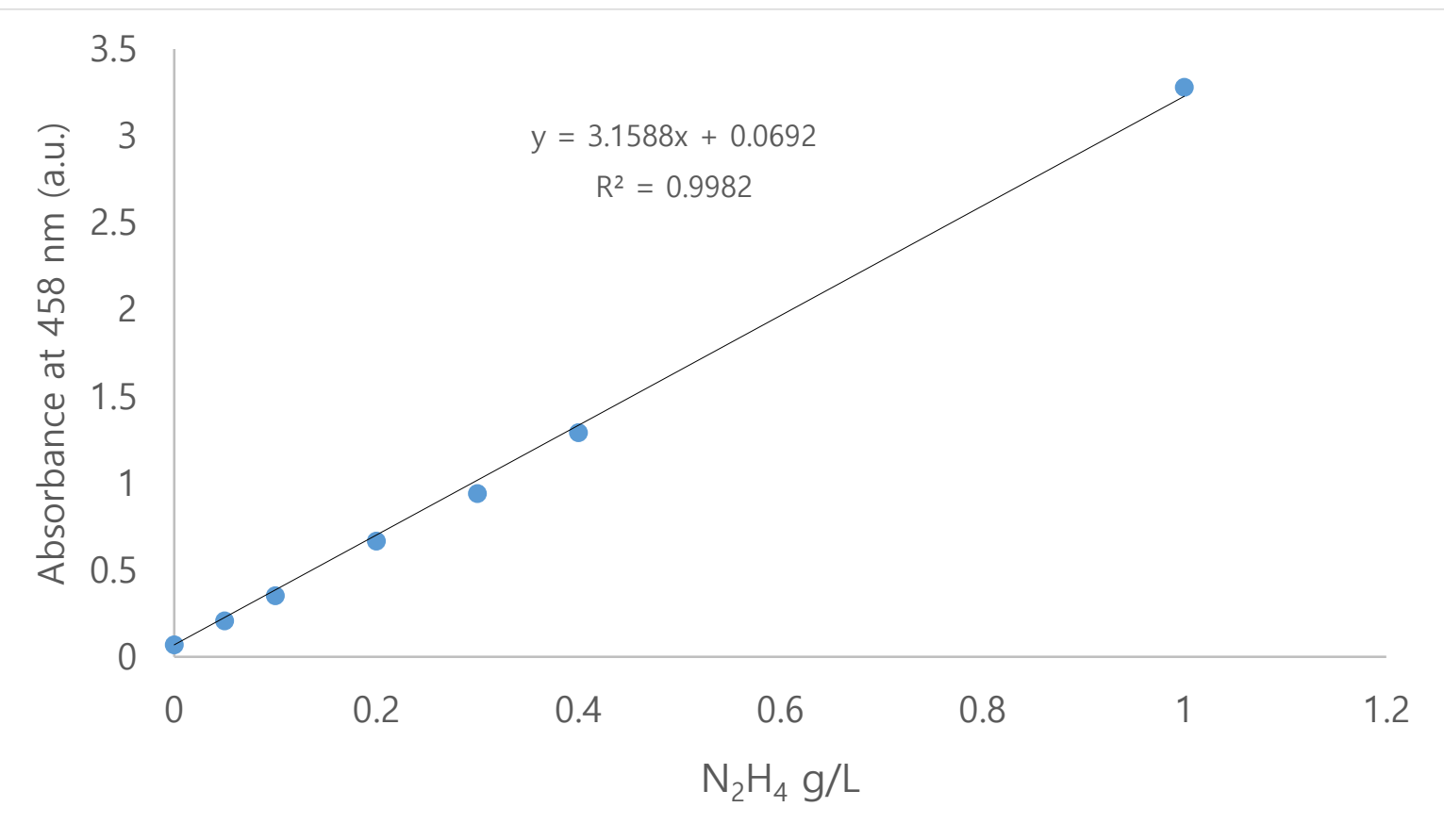

Figure S12. Calibration curve for $\mathrm{N}_{2} \mathrm{H}_{4}$ UV-VIS quantification

Table S1. Yields of $\mathrm{NH}_{3}$ by protonation of complex 3

\begin{tabular}{|c|c|c|c|c|c|c|c|}
\hline Run & Solvent & Proton source & Reductant & $\mathrm{NH}_{3} / \mathrm{Ti}$ & average $\left(\mathrm{NH}_{3} / \mathrm{Ti}\right)$ & $\mathrm{N}_{2} \mathrm{H}_{4} / \mathrm{Ti}$ & average $\left(\mathrm{N}_{2} \mathrm{H}_{4} / \mathrm{Ti}\right)$ \\
\hline 1 & $\mathrm{Et}_{2} \mathrm{O}$ & $\mathrm{PCy}_{3} \mathrm{HCl}$ & $\mathrm{KC}_{8}$ & 0.75 & \multirow{3}{*}{0.66} & 0.01 & \multirow{3}{*}{0.008} \\
\hline 2 & $\mathrm{Et}_{2} \mathrm{O}$ & $\mathrm{PCy}_{3} \mathrm{HCl}$ & $\mathrm{KC}_{8}$ & 0.62 & & 0.01 & \\
\hline 3 & $\mathrm{Et}_{2} \mathrm{O}$ & $\mathrm{PCy}_{3} \mathrm{HCl}$ & $\mathrm{KC}_{8}$ & 0.62 & & 0.004 & \\
\hline 4 & $\mathrm{Et}_{2} \mathrm{O}$ & $\mathrm{PCy}_{3} \mathrm{HI}$ & $\mathrm{KC}_{8}$ & 1.85 & \multirow{3}{*}{1.54} & 0.01 & \multirow{3}{*}{0.009} \\
\hline 5 & $\mathrm{Et}_{2} \mathrm{O}$ & $\mathrm{PCy}_{3} \mathrm{HI}$ & $\mathrm{KC}_{8}$ & 1.73 & & 0.01 & \\
\hline 6 & $\mathrm{Et}_{2} \mathrm{O}$ & $\mathrm{PCy}_{3} \mathrm{HI}$ & $\mathrm{KC}_{8}$ & 1.04 & & 0.007 & \\
\hline $7^{a}$ & $\mathrm{Et}_{2} \mathrm{O}$ & $\mathrm{PCy}_{3} \mathrm{HI}$ & $\mathrm{KC}_{8}$ & 0.0 & \multirow{3}{*}{0.0} & 0.0 & \multirow{3}{*}{0.0} \\
\hline $8^{a}$ & $\mathrm{Et}_{2} \mathrm{O}$ & $\mathrm{PCy}_{3} \mathrm{HI}$ & $\mathrm{KC}_{8}$ & 0.0 & & 0.0 & \\
\hline $9^{a}$ & $\mathrm{Et}_{2} \mathrm{O}$ & $\mathrm{PCy}_{3} \mathrm{HI}$ & $\mathrm{KC}_{8}$ & 0.0 & & 0.0 & \\
\hline 10 & pentane & $\mathrm{PCy}_{3} \mathrm{HI}$ & $\mathrm{KC}_{8}$ & 0.23 & \multirow{3}{*}{0.29} & 0.03 & \multirow{3}{*}{0.01} \\
\hline 11 & pentane & $\mathrm{PCy}_{3} \mathrm{HI}$ & $\mathrm{KC}_{8}$ & 0.34 & & 0.001 & \\
\hline 12 & pentane & $\mathrm{PCy}_{3} \mathrm{HI}$ & $\mathrm{KC}_{8}$ & 0.29 & & 0 & \\
\hline 13 & toluene & $\mathrm{PCy}_{3} \mathrm{HI}$ & $\mathrm{KC}_{8}$ & 0.22 & \multirow{3}{*}{0.25} & 0.0004 & \multirow{3}{*}{0.002} \\
\hline 14 & toluene & $\mathrm{PCy}_{3} \mathrm{HI}$ & $\mathrm{KC}_{8}$ & 0.29 & & 0.004 & \\
\hline 15 & toluene & $\mathrm{PCy}_{3} \mathrm{HI}$ & $\mathrm{KC}_{8}$ & 0.23 & & 0.0003 & \\
\hline 16 & $\mathrm{Et}_{2} \mathrm{O}$ & $\mathrm{HBAr}_{4}^{\mathrm{F}} \cdot 2 \mathrm{Et}_{2} \mathrm{O}$ & $\mathrm{KC}_{8}$ & 0.92 & \multirow{3}{*}{0.88} & 0.00008 & \multirow{3}{*}{0.007} \\
\hline 17 & $\mathrm{Et}_{2} \mathrm{O}$ & $\mathrm{HBAr}_{4} \mathrm{~F}_{4} \cdot 2 \mathrm{Et}_{2} \mathrm{O}$ & $\mathrm{KC}_{8}$ & 1.1 & & 0.01 & \\
\hline 18 & $\mathrm{Et}_{2} \mathrm{O}$ & $\mathrm{HBAr}_{4}^{\mathrm{F}} \cdot 2 \mathrm{Et}_{2} \mathrm{O}$ & $\mathrm{KC}_{8}$ & 0.63 & & 0.01 & \\
\hline 19 & $\mathrm{Et}_{2} \mathrm{O}$ & $\mathrm{HCl}$ & $\mathrm{KC}_{8}$ & 0.17 & \multirow{3}{*}{0.11} & 0.001 & \multirow{3}{*}{0.004} \\
\hline 20 & $\mathrm{Et}_{2} \mathrm{O}$ & $\mathrm{HCl}$ & $\mathrm{KC}_{8}$ & 0.15 & & 0.01 & \\
\hline 21 & $\mathrm{Et}_{2} \mathrm{O}$ & $\mathrm{HCl}$ & $\mathrm{KC}_{8}$ & 0.02 & & 0.001 & \\
\hline 22 & $\mathrm{Et}_{2} \mathrm{O}$ & $\mathrm{PCy}_{3} \mathrm{HBAr}_{4}{ }_{4}$ & $\mathrm{KC}_{8}$ & 0.92 & \multirow{3}{*}{0.83} & 0.0002 & \multirow{3}{*}{0.0006} \\
\hline 23 & $\mathrm{Et}_{2} \mathrm{O}$ & $\mathrm{PCy}_{3} \mathrm{HBAr}_{4}{ }_{4}$ & $\mathrm{KC}_{8}$ & 0.81 & & 0.001 & \\
\hline 24 & $\mathrm{Et}_{2} \mathrm{O}$ & $\mathrm{PCy}_{3} \mathrm{HBAr}_{4}{ }_{4}$ & $\mathrm{KC}_{8}$ & 0.77 & & 0.0005 & \\
\hline
\end{tabular}

${ }^{a}$ Potassium ethoxide was added as an additive (1 eq.) 


\section{1,3,5-trimethoxybenzene}

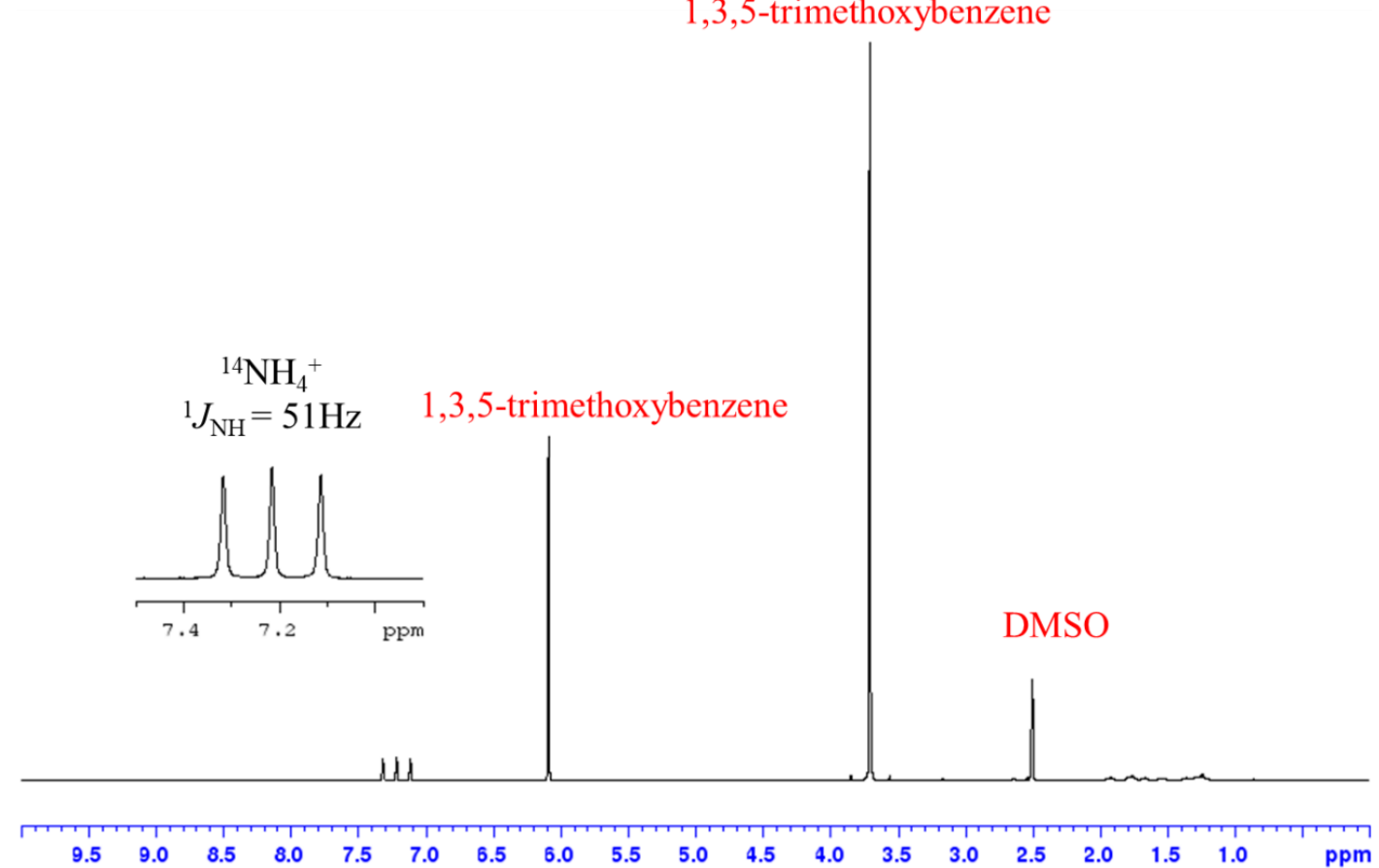

Figure S13. ${ }^{1} \mathrm{H}$ NMR spectrum of ${ }^{14} \mathrm{NH}_{4}{ }^{+}\left(7.22 \mathrm{ppm}\right.$, triplet, $\left.{ }^{1} J_{\mathrm{NH}}=51 \mathrm{~Hz}\right)$ obtained from the reduction of 3 in the presence of $\mathrm{PCy}_{3} \mathrm{HI} / \mathrm{KC}_{8}$ (Table 1, entry 2). The yield was determined using 1,3,5-trimethoxybenzene as an internal standard.

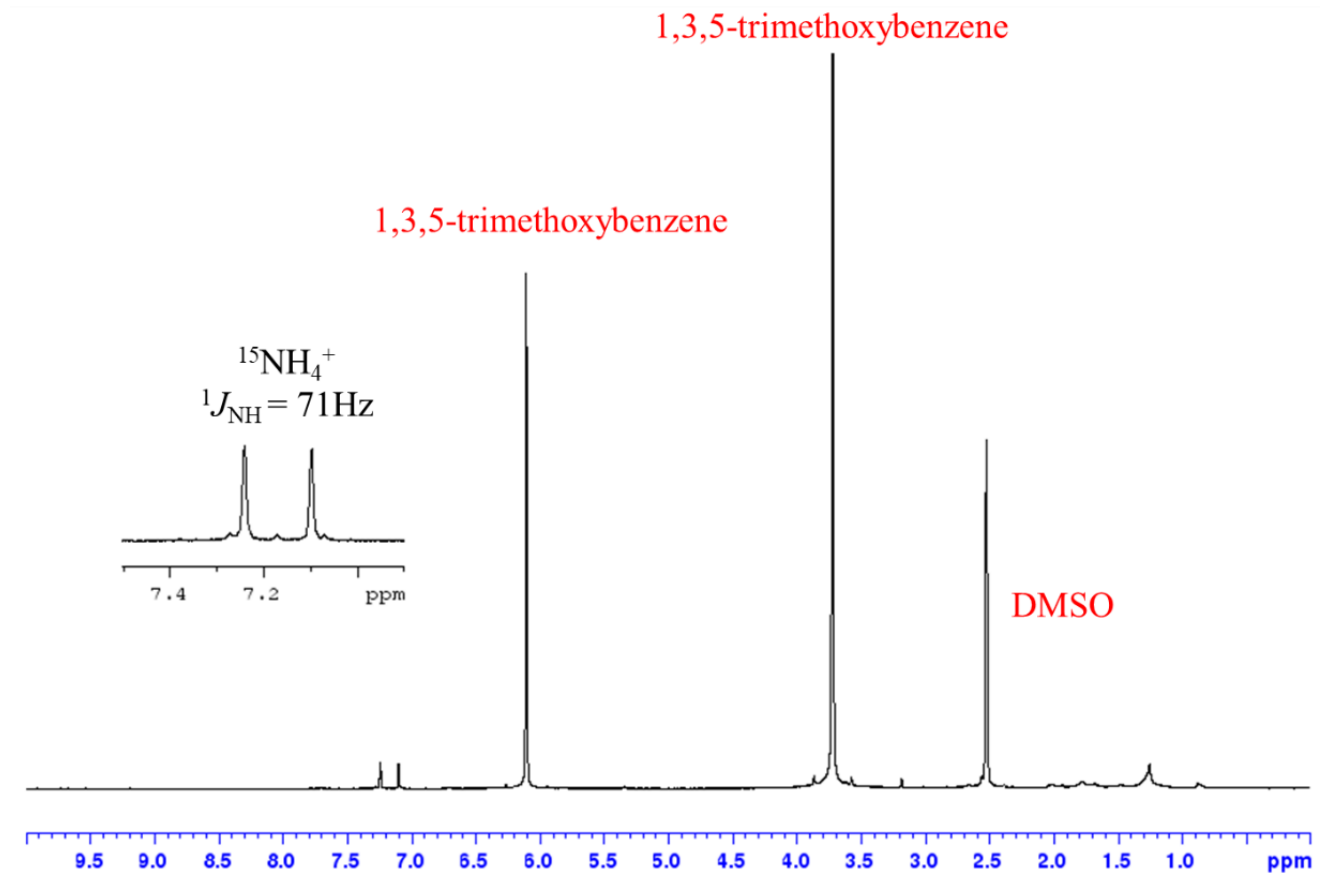

Figure S14. ${ }^{1} \mathrm{H}$ NMR spectrum of ${ }^{15} \mathrm{NH}_{4}{ }^{+}\left(7.15 \mathrm{ppm}\right.$, doublet, $\left.{ }^{1} J_{\mathrm{NH}}=71 \mathrm{~Hz}\right)$ obtained from the reduction of 3${ }^{15} \mathbf{N}_{2}$ in the presence of $\mathrm{PCy}_{3} \mathrm{HI} / \mathrm{KC}_{8}(150 / 150$ eq.). The yield was determined using 1,3,5-trimethoxybenzene as an internal standard. 


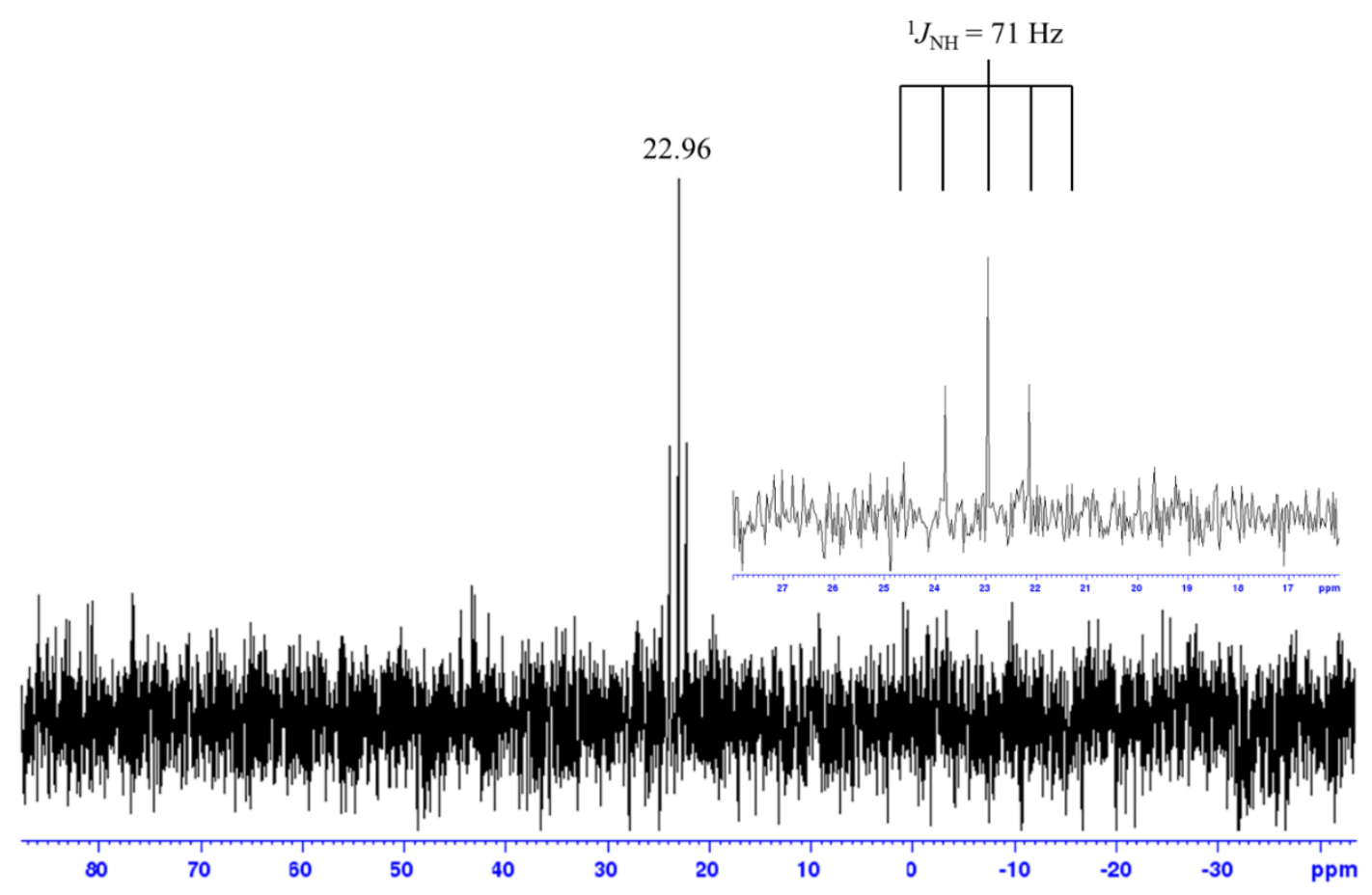

Figure S15. ${ }^{15} \mathrm{~N}$ NMR spectrum of ${ }^{15} \mathrm{NH}_{4}{ }^{+}\left(22.96 \mathrm{ppm}\right.$, quintet, $\left.{ }^{1} J_{\mathrm{NH}}=71 \mathrm{~Hz}\right)$ obtained from the reduction of 3${ }^{15} \mathbf{N}_{2}$ in the presence of $\mathrm{PCy}_{3} \mathrm{HI} / \mathrm{KC}_{8}(150 / 150$ eq.).

$\mathrm{H}_{2}$ detection experiment in the protonation of $\mathbf{3}$

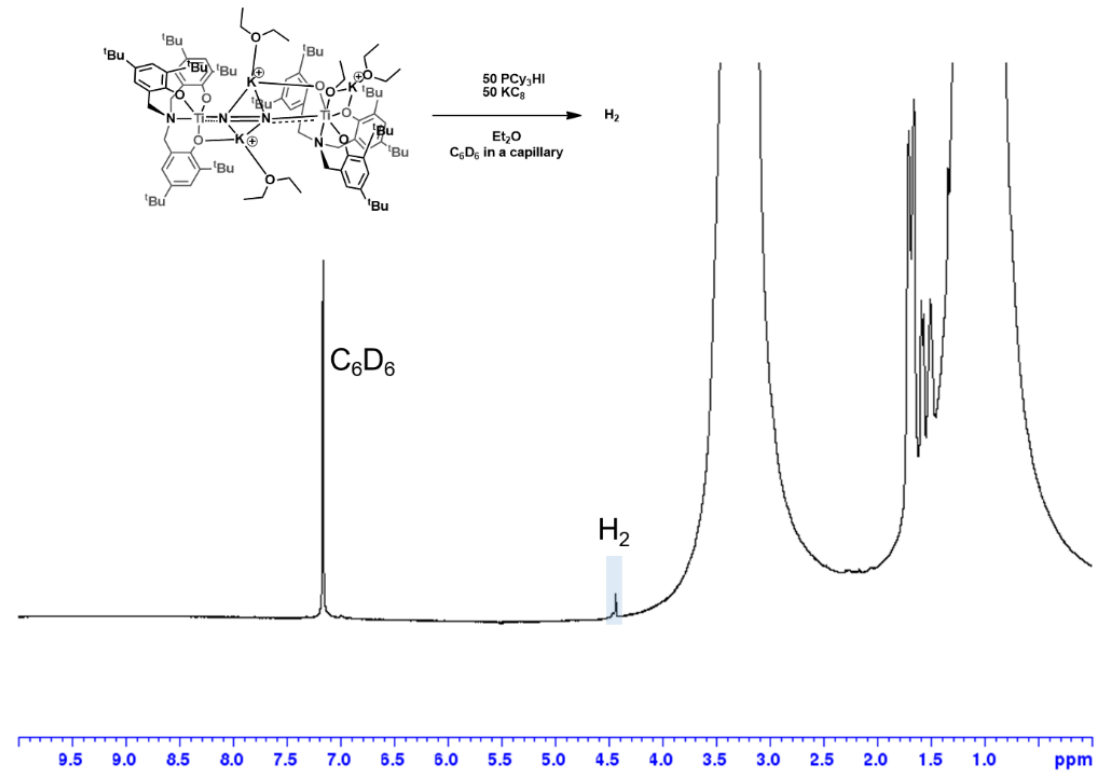

Figure S16. ${ }^{1} \mathrm{H}$ NMR spectrum of the reaction mixture of 3 and $\mathrm{PCy} 3 \mathrm{HI}$ (50 eq.) and $\mathrm{KC}_{8}(50$ eq.).

Complex 3 (2.0 mg, $1.1 \mu \mathrm{mol}, 1.0$ equiv.), $\mathrm{KC}_{8}(7.6 \mathrm{mg}, 57.0 \mu \mathrm{mol}, 50.0$ equiv.), and PCy $\mathrm{HI}(23.1 \mathrm{mg}, 57.0 \mu \mathrm{mol}$, 50.0 equiv.) were added to a JY NMR tube, followed by the addition of pre-cooled dry diethyl ether $(1.5 \mathrm{~mL},-$ $78^{\circ} \mathrm{C}$ ) and a capillary that contains $\mathrm{C}_{6} \mathrm{D}_{6}$. The mixture was left to warm to room temperature for 48 hours. $\mathrm{H}_{2}$ was detected by ${ }^{1} \mathrm{H}$ NMR. ${ }^{1} \mathrm{H}$ NMR $\left(500 \mathrm{MHz}, \mathrm{C}_{6} \mathrm{D}_{6}, 23{ }^{\circ} \mathrm{C}, \delta\right): 4.47$ (s, 2H). 


\section{X-ray Crystallographic Analysis}

CCDC-2067558, 2067568, 2067560, and 2095070 contain the supplementary crystallographic data for $\mathbf{2 , 3} \mathbf{3}, \mathbf{4}$, and 5. These data can be obtained free of charge via www.ccdc.cam.ac.uk/cgi-bin/catreq.cgi (or from the Cambridge Crystallographic Data Centre, 12, Union Road, Cambridge CB21EZ, UK; fax (+44) 1223-336-033; or deposit@ccdc.cam.ac.uk).

\section{General information}

Suitable crystals were coated with Paratone-N oil and the diffraction data measured at $100 \mathrm{~K}$ using either synchrotron radiation on the 2D beamline at the Pohang Accelerator Laboratory, Korea (for $\mathbf{4}$ and $\mathbf{5}$ ) or graphitemonochromated Mo K $\alpha(\lambda=0.71073 \AA$ ) radiation on a Bruker Venture CMOS diffractometer (for 2 and $\mathbf{3}$ ). Using Olex 2 software, ${ }^{11}$ the structure was solved by ShelXT structure solution program ${ }^{12}$ using Intrinsic Phasing and refined by ShelXL refinement package ${ }^{13}$ using least-squares minimization. All the non-hydrogen atoms were refined anisotropically and all hydrogen atoms were added to their geometrically ideal positions.

A light blue block crystal of $2\left(0.2 \times 0.05 \times 0.05 \mathrm{~mm}^{3}\right)$ was picked up with Paratone-N oil and the diffraction data were collected using a Bruker APEX-II CCD diffractometer. The crystal was kept at $100 \mathrm{~K}$ during data collection.

A brownish-purple block crystal of $\mathbf{3}\left(0.2 \times 0.1 \times 0.05 \mathrm{~mm}^{3}\right)$ was picked up with Paratone-N oil and the diffraction data were collected using a Bruker APEX-II CCD diffractometer. The crystal was kept at $100 \mathrm{~K}$ during data collection.

A colorless needle crystal of $4\left(0.15 \times 0.02 \times 0.01 \mathrm{~mm}^{3}\right)$ was picked up with Paratone-N oil and the diffraction data were collected at $100 \mathrm{~K}$ with synchrotron radiation on a $2 \mathrm{D}$ beamline at the Pohang Accelerator Laboratory, Korea.

A brownish violet block crystal of $5\left(0.04 \times 0.04 \times 0.03 \mathrm{~mm}^{3}\right)$ was picked up with Paratone-N oil and the diffraction data were collected at $100 \mathrm{~K}$ with synchrotron radiation on a $2 \mathrm{D}$ beamline at the Pohang Accelerator Laboratory, Korea.

Crystal data and structure refinement for $\mathbf{2}$

$\begin{array}{ll}\text { Identification code } & \mathrm{Cc} \\ \text { Empirical formula } & \mathrm{C}_{49} \mathrm{H}_{76} \mathrm{NO}_{4} \mathrm{Ti} \\ \text { Formula weight } & 791.00 \\ \text { Temperature/K } & 100 \\ \text { Crystal system } & \text { monoclinic } \\ \text { Space group } & \mathrm{Cc} \\ \mathrm{a} / \AA & 21.293(2) \\ \mathrm{b} / \AA & 18.6898(18) \\ \mathrm{c} / \AA & 12.1854(14) \\ \alpha / /^{\circ} & 90 \\ \beta /{ }^{\circ} & 108.031(4) \\ \gamma /{ }^{\circ} & 90 \\ \mathrm{Volume} / \AA^{3} & 4611.2(9) \\ \mathrm{Z} & 4 \\ \rho \mathrm{calcg} / \mathrm{cm}^{3} & 1.139 \\ \mu / \mathrm{mm}^{-1} & 0.227\end{array}$


$\mathrm{F}(000)$

Crystal size $/ \mathrm{mm}^{3}$

Radiation

$2 \Theta$ range for data collection/ ${ }^{\circ}$

Index ranges

Reflections collected

Independent reflections

Data/restraints/parameters

Goodness-of-fit on $\mathrm{F}^{2}$

Final $\mathrm{R}$ indexes $[\mathrm{I}>=2 \sigma(\mathrm{I})]$

Final $\mathrm{R}$ indexes [all data]

Largest diff. peak/hole / e $\AA^{-3}$

Flack parameter

Crystal data and structure refinement for $\mathbf{3}$

Identification code

Empirical formula

Formula weight

Temperature/K

Crystal system

Space group

$\mathrm{a} / \AA$

$\mathrm{b} / \AA$

$\mathrm{c} / \AA$

$\alpha /^{\circ}$

$\beta /{ }^{\circ}$

Volume/ $\AA^{3}$

pcalcg/ $/ \mathrm{cm}^{3}$

$\mu / \mathrm{mm}^{-1}$

$\mathrm{F}(000)$

Crystal size $/ \mathrm{mm}^{3}$

Radiation

$2 \Theta$ range for data collection $/{ }^{\circ}$

Index ranges

Reflections collected

Independent reflections

Data/restraints/parameters

Goodness-of-fit on $\mathrm{F}^{2}$

Final $\mathrm{R}$ indexes $[\mathrm{I}>=2 \sigma(\mathrm{I})]$

Final $\mathrm{R}$ indexes [all data]

Largest diff. peak/hole / e $\AA^{-3}$
1724.0

$0.2 \times 0.05 \times 0.05$

$\operatorname{MoK} \alpha(\lambda=0.71073)$

2.966 to 59.486

$-29 \leq \mathrm{h} \leq 29,-25 \leq \mathrm{k} \leq 26,-16 \leq 1 \leq 16$

38245

$12708\left[\mathrm{R}_{\text {int }}=0.0516, \mathrm{R}_{\text {sigma }}=0.0681\right]$

$12708 / 26 / 516$

1.046

$\mathrm{R}_{1}=0.0592, \mathrm{wR}_{2}=0.1490$

$\mathrm{R}_{1}=0.0711, \mathrm{wR}_{2}=0.1570$

$1.23 /-0.57$

$-0.015(12)$

P-1

$\mathrm{C}_{108} \mathrm{H}_{177} \mathrm{~K}_{3} \mathrm{~N}_{4} \mathrm{O}_{11} \mathrm{Ti}_{2}$

1920.63

100

triclinic

P-1

$14.8535(14)$

$15.6140(16)$

$29.070(3)$

$77.033(3)$

$84.902(3)$

$61.786(3)$

5788.3(10)

1.102

0.299

2084.0

$0.2 \times 0.1 \times 0.05$

$\operatorname{MoK} \alpha(\lambda=0.71073)$

2.876 to 49.424

$-17 \leq \mathrm{h} \leq 17,-18 \leq \mathrm{k} \leq 18,-34 \leq 1 \leq 34$

97594

$19745\left[\mathrm{R}_{\text {int }}=0.0618, \mathrm{R}_{\text {sigma }}=0.0503\right]$

$19745 / 1250 / 1199$

1.043

$\mathrm{R}_{1}=0.0747, \mathrm{wR}_{2}=0.2101$

$\mathrm{R}_{1}=0.0933, \mathrm{wR}_{2}=0.2244$

$1.43 /-0.95$

Crystal data and structure refinement for $\mathbf{4}$

Identification code
Empirical formula
Formula weight
Temperature $/ \mathrm{K}$
Crystal system
Space group
$\mathrm{a} / \AA$
$\mathrm{b} / \AA$
$\mathrm{c} / \AA$
$\alpha /^{\circ}$
$\beta /{ }^{\circ}$
$\gamma /{ }^{\circ}$
Volume $/ \AA^{3}$
$\mathrm{Z}$
$\rho$ calcg $/ \mathrm{cm}^{3}$

P21n_b

$\mathrm{C}_{66} \mathrm{H}_{84} \mathrm{ClNO}_{3} \mathrm{Ti}$

1022.69

100

monoclinic

$\mathrm{P} 21 / \mathrm{n}$

9.5830(19)

26.357(5)

$24.442(5)$

90

96.73(3)

90

6131(2)

4

1.108 
$\mu / \mathrm{mm}^{-1}$

$\mathrm{F}(000)$

Crystal size $/ \mathrm{mm}^{3}$

Radiation

$2 \Theta$ range for data collection ${ }^{\circ}$

Index ranges

Reflections collected

Independent reflections

Data/restraints/parameters

Goodness-of-fit on $\mathrm{F}^{2}$

Final R indexes $[\mathrm{I}>=2 \sigma(\mathrm{I})]$

Final $\mathrm{R}$ indexes [all data]

Largest diff. peak/hole / e $\AA^{-3}$
0.226

2200.0

$0.15 \times 0.02 \times 0.01$

synchrotron $(\lambda=0.70000)$

3.09 to 49.426

$-11 \leq \mathrm{h} \leq 11,-31 \leq \mathrm{k} \leq 31,-28 \leq 1 \leq 28$

38921

$10458\left[\mathrm{R}_{\text {int }}=0.1174, \mathrm{R}_{\text {sigma }}=0.1036\right]$

$10458 / 0 / 667$

0.874

$\mathrm{R}_{1}=0.0528, \mathrm{wR}_{2}=0.1176$

$\mathrm{R}_{1}=0.1066, \mathrm{wR}_{2}=0.1323$

$0.43 /-0.39$

Crystal data and structure refinement for $\mathbf{5}$

Identification code

Empirical formula

Formula weight

Temperature/K

Crystal system

Space group

$\mathrm{a} / \AA$

$\mathrm{b} / \AA$

$\mathrm{c} / \AA$

$\alpha /^{\circ}$

$\beta /{ }^{\circ}$

$\gamma /{ }^{\circ}$

Volume $/ \AA^{3}$

Z

pcalcg/cm ${ }^{3}$

$\mu / \mathrm{mm}^{-1}$

$\mathrm{F}(000)$

Crystal size $/ \mathrm{mm}^{3}$

Radiation

$2 \Theta$ range for data collection $/{ }^{\circ}$

Index ranges

Reflections collected

Independent reflections

Data/restraints/parameters

Goodness-of-fit on $\mathrm{F}^{2}$

Final $R$ indexes $[\mathrm{I}>=2 \sigma(\mathrm{I})]$

Final $\mathrm{R}$ indexes [all data]

Largest diff. peak/hole / e $\AA^{-3}$

Flack parameter
R3c

C68H89KNO4Ti

1071.40

100

trigonal

R3c

14.507(2)

$14.507(2)$

49.854(10)

90

90

120

$9086(3)$

6

1.175

0.257

3462.0

$0.04 \times 0.04 \times 0.03$

synchrotron $(\lambda=0.70000)$

3.63 to 56.578

$-19 \leq \mathrm{h} \leq 19,-19 \leq \mathrm{k} \leq 19,-66 \leq 1 \leq 66$

22118

$5028\left[\mathrm{R}_{\text {int }}=0.0952, \mathrm{R}_{\text {sigma }}=0.0615\right]$

$5028 / 235 / 252$

1.022

$\mathrm{R}_{1}=0.0739, \mathrm{wR}_{2}=0.1863$

$\mathrm{R}_{1}=0.0793, \mathrm{wR}_{2}=0.1920$

$0.65 /-0.63$

$0.051(15)$ 


\section{DFT Calculation}

General information

The DFT calculations were performed using Gaussian $16^{14}$ at the clusters at Korea Institute of Science and Technology Information (KISTI) computational resource. Geometry optimizations of all compounds were carried out using the atomic coordinates derived from energy minimized or crystal structures. No constraints were forced on the geometry optimization. The ${ }^{t} \mathrm{Bu}$ groups of the para position of ligand $\mathbf{L} 1$ were replaced by hydrogen atoms for computational efficiency. All geometries were optimized using the M06L ${ }^{15} / \mathrm{BSI} / \mathrm{BSII}$ and under standard convergence criteria with the following basis sets, which include Struttgart-Dresen (SDD) quasirelativistic pseudopotentials on $\mathrm{Ti}$ (10 core electrons), K (10) with their associated basis sets (Ti: $(8 \mathrm{~s} 7 \mathrm{p} 6 \mathrm{~d}) /[6 \mathrm{~s} 5 \mathrm{p} 3 \mathrm{~d}])^{16}$ augmented by polarization functions (Ti: f, 1.506), ${ }^{17}(\mathrm{~K}: \mathrm{d}, 1.000),{ }^{17 \mathrm{a}, 18}$ and $6-31 \mathrm{G}(\mathrm{d}, \mathrm{p}){ }^{19}$ on $\mathrm{H}, \mathrm{C}, \mathrm{N}$, and O. For all BS I-optimized geometries, single-point energy calculations for solvation energies were performed with BS II, in which SDD pseudopotentials are used for Ti (10) with the associated SDD basis set augmented by (2f1g) functions ${ }^{20}$ and for the other atoms described by $6-311 \mathrm{G}+(2 \mathrm{~d}, \mathrm{p})$ basis sets, ${ }^{21}$ using solvent model density (SMD) method of Cramer and Truhlar. ${ }^{16 c} \mathrm{G}^{\circ}{ }_{\text {solvent }}(\mathrm{BSII} / \mathrm{BSI})$ values were combined with thermochemical corrections calculated via frequency analyses at M06L/BSI level in the gas phase without scaling to produce improved estimates in the text and schemes $\left(\mathrm{G}_{\text {solvent }}^{\circ}(\mathrm{BSII} / \mathrm{BSI})=\mathrm{G}^{\circ}\right.$ gas $(\mathrm{BSI})+\left(\mathrm{E}_{\text {solvent }}^{\circ}(\mathrm{BSII})-\mathrm{E}_{\text {gas }}^{\circ}(\mathrm{BSI})\right)$.

(a)

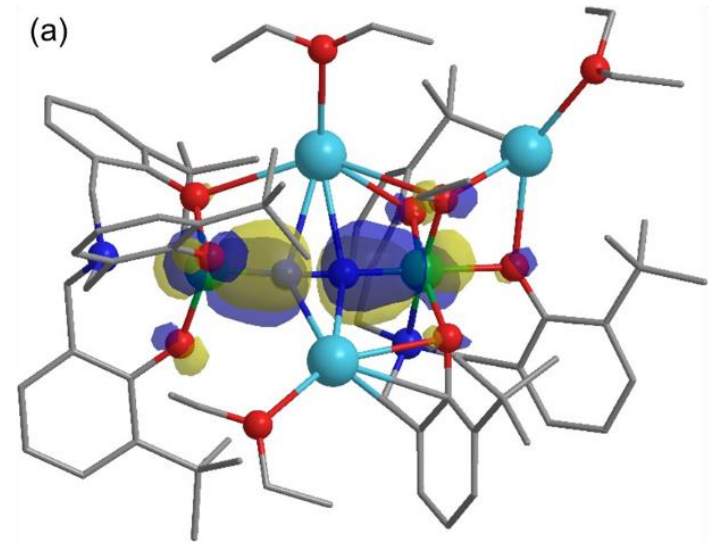

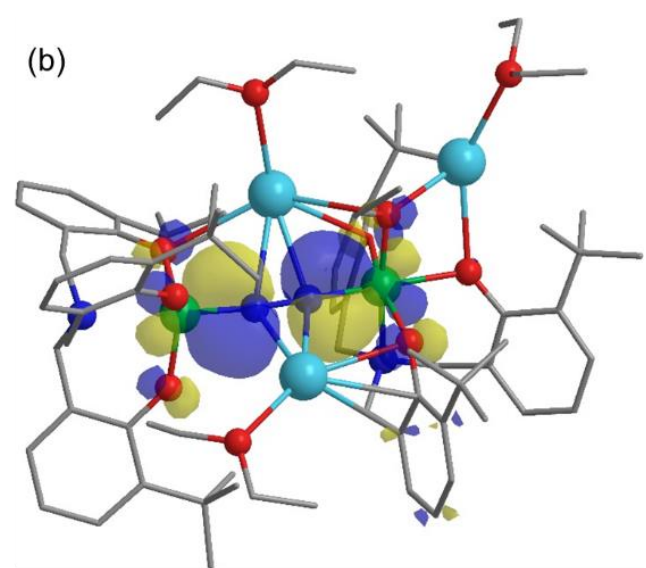

Figure S17. Molecular orbitals showing the two orthogonal $\mathrm{Ti}(\mathrm{d} \pi) \rightarrow \mathrm{N}_{2}\left(\pi^{*}\right)$ back-bonding interactions. (a) HOMO-1 of $\mathbf{3}_{\mathbf{D F T}}$; (b) HOMO of $\mathbf{3}_{\mathbf{D F T}}$. Hydrogen atoms are omitted for clarity. Isocontour level: 0.03. Color: titanium (green); nitrogen (blue); oxygen (red); carbon (gray); potassium (cyan). 
Metric comparison of X-ray structure and DFT optimized structures

Table S2. Selected bond lengths $(\AA)$, angles $\left({ }^{\circ}\right)$, and N-N frequencies $\left(\mathrm{cm}^{-1}\right)$ comparison for $3_{\text {DFT }}$ and 3.

\begin{tabular}{ccc} 
& 3DFT (M06L) & crystal structure 3 \\
\hline bond length $(\AA)$ & & \\
Ti1-O1 & 1.981 & $1.984(2)$ \\
Ti1-N1 & 2.343 & $2.258(2)$ \\
Ti1-N4 & 1.783 & $1.791(3)$ \\
N3-N4 & 1.277 & $1.288(4)$ \\
Ti2-N3 & 1.787 & $1.803(3)$ \\
Ti2-N2 & 2.265 & $2.258(5)$ \\
Ti2-O5 & 2.177 & $2.156(3)$ \\
\hline angle ( $\left.{ }^{\circ}\right)$ & & \\
O1-Ti1-O2 & 117.4 & $118.6(1)$ \\
N1-Ti1-N4 & 176.3 & $174.0(1)$ \\
T11-N4-N3 & 174.5 & $172.6(3)$ \\
N4-N3-Ti2 & 172.1 & $169.5(3)$ \\
N3-Ti2-N2 & 90.0 & $89.1(1)$ \\
N2-Ti2-O7 & 171.4 & $171.0(1)$ \\
O4-Ti2-O5 & 79.4 & $81.6(1)$ \\
\hline v(N-N) (cm $\left.{ }^{-1}\right)$ & 1430 & 1304 \\
Raman activity & 1611.8 &
\end{tabular}

Table S3. Selected bond lengths $(\AA)$, angles $\left({ }^{\circ}\right)$ comparison for $\mathbf{2}_{\mathrm{DFT}}$ and 2

\begin{tabular}{|c|c|c|}
\hline & 2 & crystal structure 2 \\
\hline \multicolumn{3}{|l|}{ bond length $(\AA)$} \\
\hline Ti1-O1 & 1.880 & $1.868(2)$ \\
\hline Ti1-O4 & 2.180 & $2.159(3)$ \\
\hline Ti1-N1 & 2.202 & $2.181(4)$ \\
\hline \multicolumn{3}{|l|}{ angle $\left(^{\circ}\right)$} \\
\hline N1-Ti1-O4 & 175.9 & $178.0(1)$ \\
\hline O1-Ti1-O2 & 128.6 & $126.1(1)$ \\
\hline N1-Ti1-O1 & 86.3 & $86.5(1)$ \\
\hline
\end{tabular}




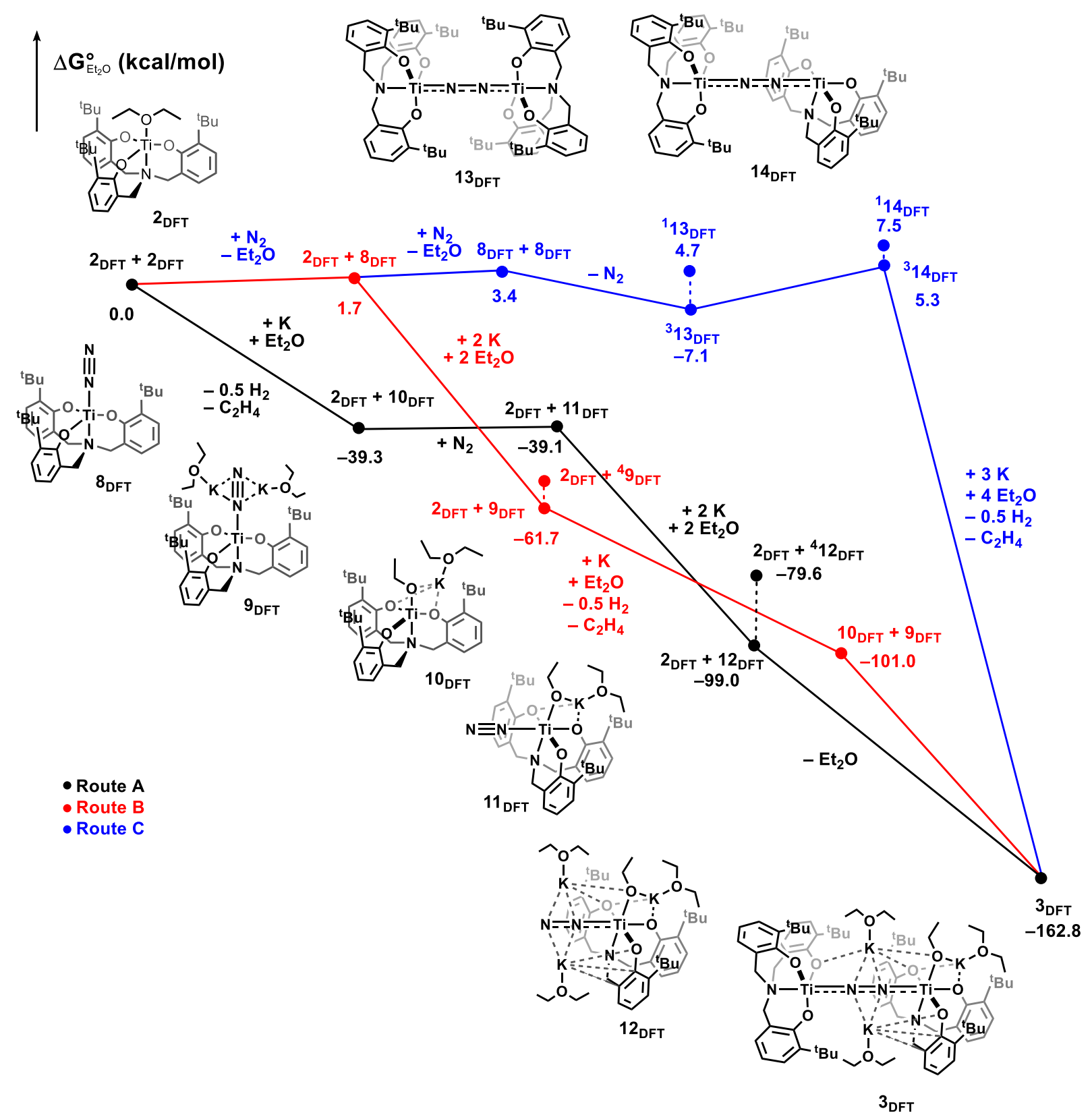

Scheme S1. Potential reaction pathways to 3 DFT from 2DFT. Ground state energies are relative to the sum of 2 equivalents of $\mathbf{2}_{\text {DFT }}$, solution ( $\left(\mathrm{Et}_{2} \mathrm{O}\right)$ phase (SMD model). Superscripts indicate spin multiplicities. $\mathbf{3}_{\text {DFT }}$ and ${ }^{4} \mathbf{1}_{\text {DFT }}$ have singlet and quartet spin states, respectively, and ${ }^{\mathbf{3}} \mathbf{1 3}_{\mathrm{DFT}}$ and ${ }^{\mathbf{3}} \mathbf{1 4}_{\mathrm{DFT}}$ have triplet spin states. Other structures have a doublet spin state. 


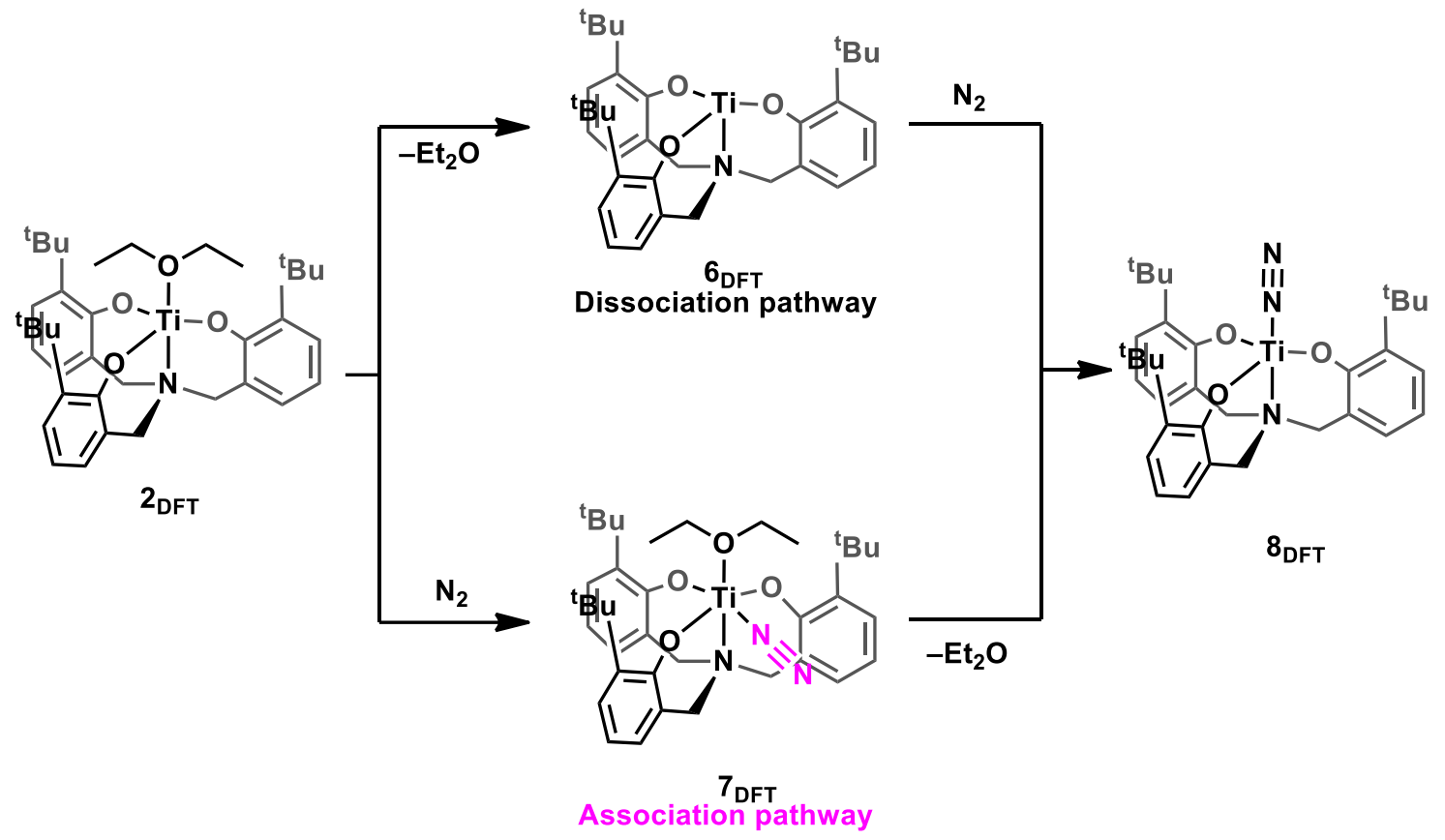

Scheme S2. Potential reaction pathways to $\mathbf{8}_{\mathrm{DFT}}$ from 2 DFT.

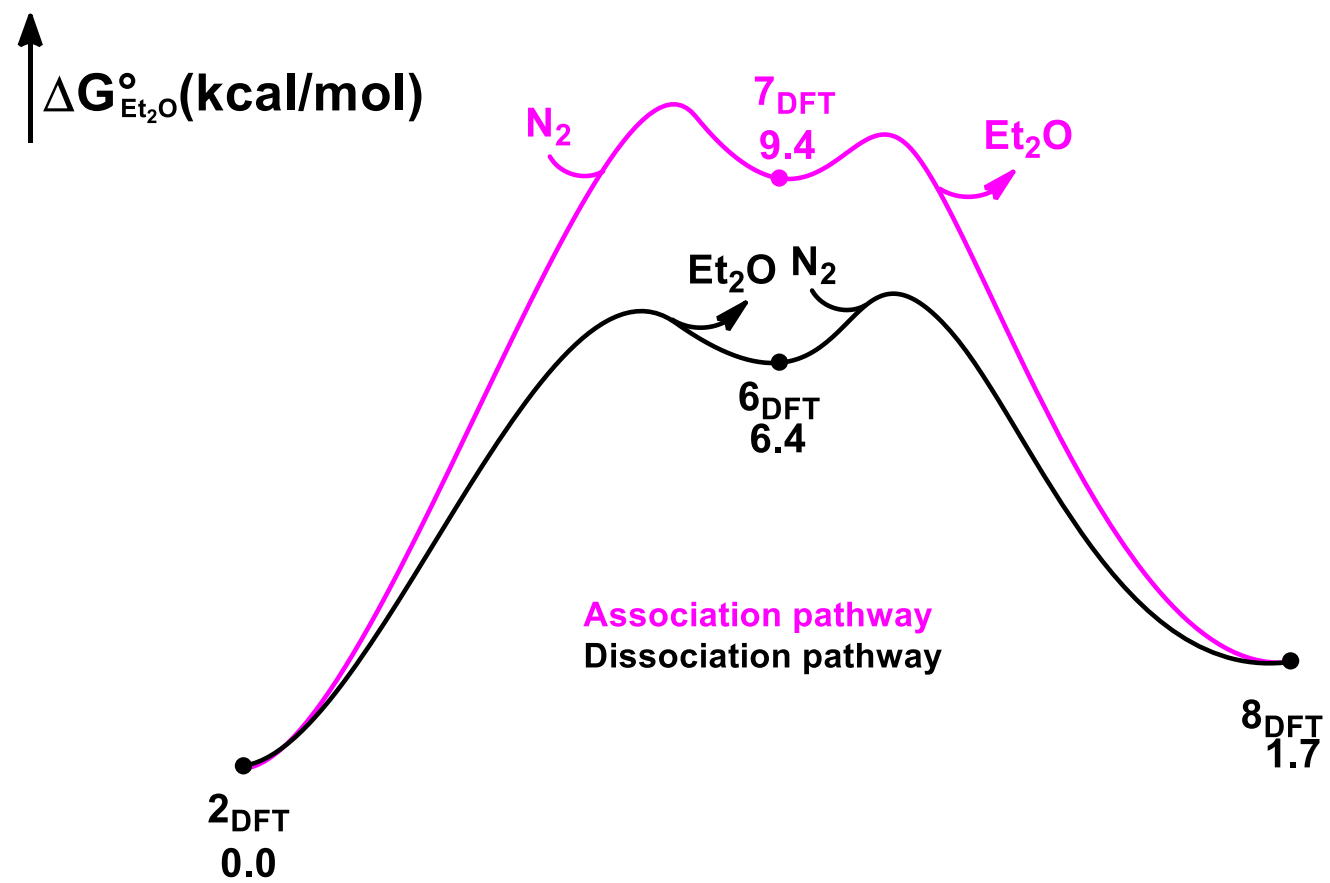

Scheme S3. Potential thermodynamic profiles from $\mathbf{2}_{\text {DFT }}$ to $\mathbf{8}_{\text {DFT }}$. Ground state energies are relative to $\mathbf{2}_{\text {DFT, }}$ solution $\left(\mathrm{Et}_{2} \mathrm{O}\right)$ phase (SMD model). Superscripts indicate spin multiplicities. 
Mayer bond order

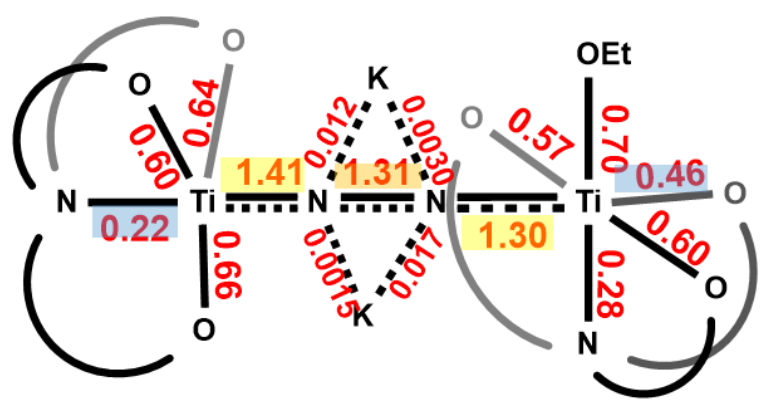

Bond length of complex 3

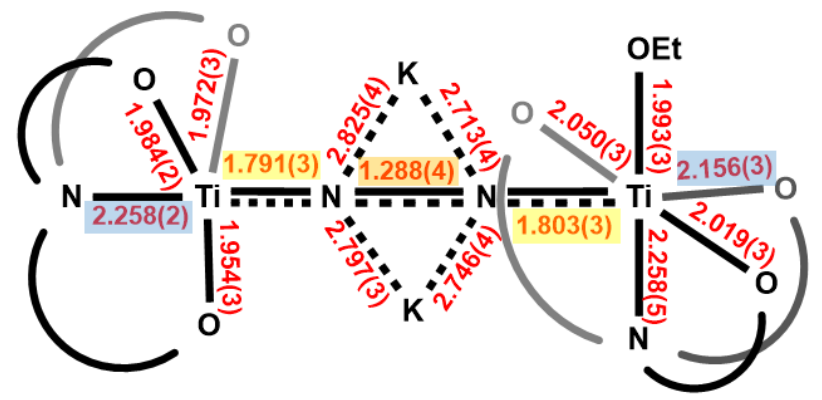

Unit: $\AA$

Figure S18. Comparisons of Mayer bond orders of 3DFT and bond lengths of complex 3.

Coordinates of optimized structures

The following optimized geometries are displayed in Cartesian coordinates (atomic unit). $\mathrm{E}^{\circ}$ represents the electronic energy of the optimized structure, and $\mathrm{G}^{\circ}$ represents the sum of electronic and thermal free energies in Hartree unit. $\mathrm{G}^{\circ}{ }_{\mathrm{E} 2 \mathrm{O}}$ values were obtained by considering solvent effects applying solvent model density (SMD) method in single-point calculations using diethyl ether as a solvent.

2 $\mathrm{DFT}$

$\mathrm{E}^{0}=-1855.349534 ; \mathrm{G}^{0}=-1854.603523 ;$

$\mathrm{E}_{\mathrm{Et} 2 \mathrm{O}}^{\mathrm{o}}=-1855.811807 ; \mathrm{G}^{\mathrm{o}} \mathrm{Et2O}=-1855.065796 ;$

Charge $=0 ;$ Multiplicity $=2$;

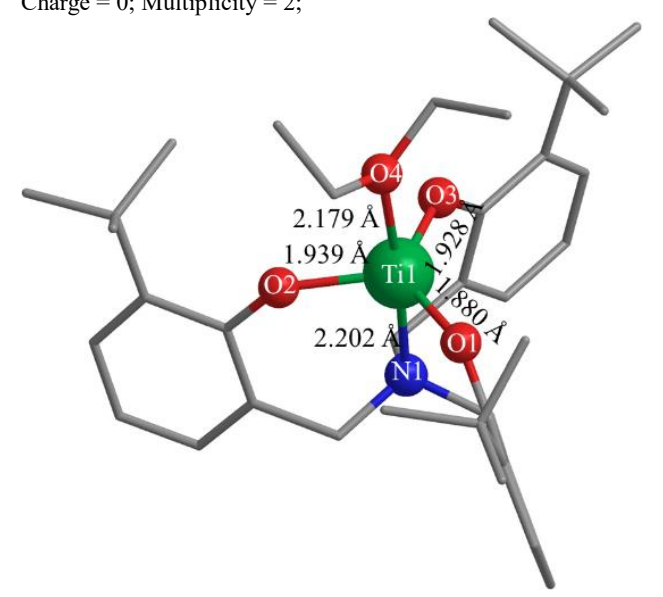

Ti 2.73287253281 .78228080841 .4962076617

O 4.53873806251 .07623071391 .4810981823 O 2.42878104863 .20081720860 .225653957 O 2.2112082460 .21944670340 .0700392437 O 1.59358247281 .16101357612 .8563181993 C 0.913171331 .98209635916 .8525080622 N 3.40383261523 .29604068222 .9483224739 C 0.6247691480 .72013562646 .3403527522 C 1.45911332492 .94802306796 .0193318642 C 5.72702850452 .37513765463 .0812151479 C 8.11890859112 .62037558582 .8835032511 C 2.97163110315 .20368392481 .3804064674 C 1.90335123337 .2820188330 .7778346338 C 8.09132451051 .82103923151 .7429929224 C 3.97026420614 .45858972762 .2055001115 C 5.7039607731 .56232380371 .9247836568 C 1.68370519572 .67065873554 .6727547148

\begin{abstract}
C 1.3653604291 .39605912274 .1596782619 C 2.23820647853 .72959564583 .7691429173 C 0.85009510330 .38244205685 .0027948667 C $1.17388325566 .604961891-0.1952497507$ C 2.23511979934 .52081565590 .3865878362 C 4.45276420292 .67148048933 .8079170211 C 6.93210038662 .899160933 .5447648565 C 2.79430640536 .57269040151 .5693838588 C $-0.46549108533 .5045154339-0.7353476549$ C $0.44934722374 .5079830575-1.4564826401$ C $6.4370675609-1.01699707610 .3433581581$ C $1.30508892825 .2315983665-0.4164430693$ C $0.6205006618-1.03838410754 .4823930022$ C $6.91060137710 .3974767494-0.0214031246$ C $1.34180334623 .8066571137-2.4922443968$ C $-0.3416998981-1.04503835263 .2858903953$ C 6.91012713841 .27771726511 .2317983507 C $1.19952065750 .3526046609-0.9494629613$ C $1.9761398052-1.6361340624 .0729302165$ C $2.554503131-1.13505556420 .4382617168$ C $6.00027295440 .9895987886-1.110053627$ C $3.0507650773-1.9577288412-0.7247456572$ C $-0.46084874455 .4652031072-2.2296776604$ C $0.0192345617-1.95067380145 .5535984374$ C $8.31093629130 .2683241598-0.6259584608$ C $-0.1655402728-0.0867287636-0.4825489447$ H 0.72724536752 .19946450747 .8998151942 H $0.2200790306-0.02886852487 .0132914642$ H 1.71469112023 .93313580556 .4054283687 H 9.06011364683 .02523738163 .242688934 H 1.76736289228 .34995063280 .9180479866 H 9.02875975981 .62245373691 .2334083697 H 4.77529667924 .05775747451 .5758972629 H 4.44366432575 .14302045522 .9287485918 H 1.47744361984 .07932213493 .0569089637 H 2.53119101914 .60638332794 .3708210005 H $0.47216129937 .1711585907-0.7993830622$ H 4.00586871321 .75514198464 .2185188197 H 4.65873091423 .33768266394 .6621103072 H 6.92625631783 .53054373124 .4320911409
\end{abstract}


H 3.37027434567 .07586159382 .34438666547 H $0.09434603742 .8376493809-0.0754198112$ H - $1.20123360454 .0345613058-0.1211531235$ H - $1.01712252072 .8912955162-1.4593377293$ H $5.4360655743-0.99855605410 .7783479124$ H $7.1150971172-1.47828044981 .0692377862$ H $6.4176995626-1.6557345494-0.5485563127$ H $1.89186837134 .5480139519-3.0813713877$ H $2.07520772863 .1456573245-2.0284123417$ Н $0.7283006253 .2214560243-3.1887486329$ H $0.0432812599-0.45536183082 .4535359052$ H - $1.3182874827-0.63732992953 .5680208826$ H - $0.4994003942-2.07367000832 .9379688363$ H $1.21615919331 .4154181968-1.1994728864$ H $1.5281980218-0.2003172169-1.8371998567$ H $2.4848253576-1.01405303563 .3326856926$ H $2.6350626042-1.73013777784 .9425114496$ H $1.8412854733-2.63783239513 .6461699652$ H $3.3412717332-1.0063119891 .187383153$ H $1.6905217792-1.59881721980 .9349433557$ H $6.31633839472 .0051028195-1.3723394153$ H $4.95560432021 .030835911-0.7958931813$ H $6.06306195740 .3792226401-2.019582398$ H $3.8373921238-1.4250797513-1.2671696768$ H $3.4781590997-2.8909848553-0.3487153767$ H $2.2559201189-2.2229113276-1.4275467823$ H $0.11064032236 .2208917486-2.7785981211$ H - $1.16970910715 .9826524557-1.5750197443$ H - $1.04524854344 .8986924413-2.9622777966$ H - $0.9544245779-1.58912186645 .9010188918$ H $0.6745116192-2.05450322596 .4244036717$ H - $0.1303710902-2.95177801845 .1365203996$ H $8.71783035641 .2379405383-0.9320459206$ H $9.0201176423-0.1938862690 .0686223992$ H $8.2651572062-0.3658879336-1.5172656003$ H - $0.9014881320 .0951007068-1.2704825337$ H $-0.2001743989-1.1530619869-0.2380395587$ H - 0.47539047210 .47649366220 .4035066402

${ }^{2} \mathbf{6}_{\mathrm{DFT}}$

$\mathrm{E}^{0}=-1621.669487 ; \mathrm{G}^{0}=-1621.055204 ;$

$\mathrm{E}^{0} \mathrm{EtOO}_{2}=-1622.074225 ; \mathrm{G}^{0} \mathrm{Et}_{2 \mathrm{O}}=-1621.459942 ;$

Charge $=0 ;$ Multiplicity $=2$

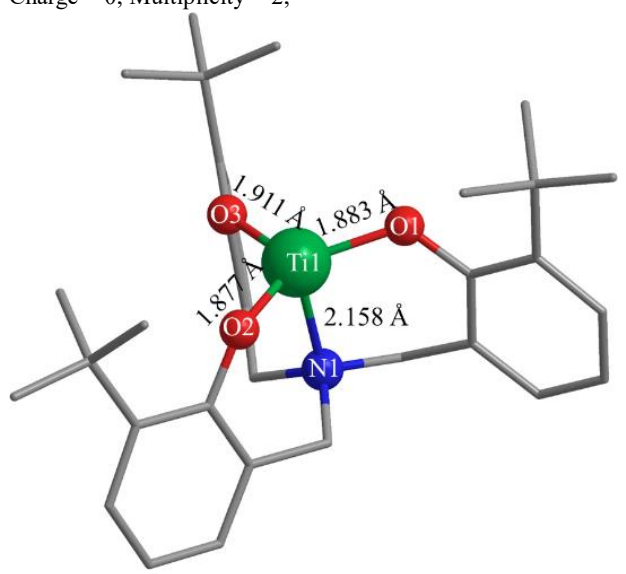

Ti $-0.3351406511-0.6065454213-0.6924672105$ O $1.3020030284-1.5899048254-0.624818451$ O $-0.3117447920 .6913849125-2.0561407387$ O $-1.6023691829-1.22446343460 .5463583623$ C $-2.1414125871-0.72569628214 .6163658775$ N 0.28743703930 .85104927040 .7726947637 C $-2.5140179354-1.91980451174 .0046527699$ C -1.58463121520 .28915377043 .8515742102$ C $2.5877790932-0.17422910890 .7975906666$ C $4.9752218664-0.01246281050 .4810460612$ C $-0.19338057172 .7081202195-0.8355818018$ C $-1.49588023064 .6191216214-1.5119304322$
C $4.883400803-0.9254533956-0.5670885156$ C 0.81199718012 .05813173810 .0637400565 C $2.5022938769-1.0958731765-0.273264315$ C -1.41704467250 .12011155232 .4788024335$ C $-1.8005393235-1.09365509231 .8714754833$ C -0.86496689741 .23419406181 .6432282682$ C - $2.3534203448-2.1478273862 .6346405619$ C $-2.01788098223 .8766720059-2.5681784514$ C $-0.72773540881 .9679145864-1.9126677966$ C 1.36621614960 .19635939861 .5803741801 C 3.82446484370 .36169875841 .1570706996 C $-0.58763405084 .030044054-0.6430193516$ C $-3.06557170860 .5465803552-3.3968386715$ C $-2.2644589371 .7349099017-3.9528665143$ C $3.0134989479-3.8154247954-1.6716583919$ C $-1.66265845572 .5443604591-2.8018686108$ C $-2.7351468386-3.48101110671 .9875069414$ C $3.5978866616-2.4752596986-2.1426858125$ C $-1.15723279071 .2290402817-4.8893774388$ C $-3.7869011218-3.26328838950 .8897174209$ C $3.6710556748-1.4864041014-0.975670201$ C - $1.4849128723-4.15058839031 .3953179932$ C $2.7354214835-1.8956893419-3.2750568903$ C $-3.22862656432 .5761579212-4.7924265675$ C -3.3337064788 -4.4541939611 3.00577187 C $4.9816936894-2.762546062-2.7305017485$ H - $2.2791925328-0.59676985255 .6854219634$ H - $2.9382106716-2.70386985214 .6231792042$ H - 1.27740812711 .22667846454 .3114493475 H 5.93925379430 .40090607340 .7609261402 H - $1.80459480625 .6500327605-1.3689486529$ H $5.7949847585-1.2037358138-1.0857764255$ H $1.6858359291 .7186908876-0.5059936405$ H 1.17067432482 .77554716980 .8187855739 H - 1.62911008051 .64024984870 .9661138731 H -0.54746917082 .06276323592 .2971334546$ H - $2.73164262524 .3562657938-3.2299742742$ H $0.9019467594-0.68992839882 .0332961044$ H 1.64122944250 .87039891832 .407233229 H 3.8715454321 .07257988251 .980593287 H - 0.17246408534 .59156004880 .1919841873 H $-3.86905086810 .8890433951-2.7361513883$ H - $3.5244572382-0.016779353-4.2170843744$ H - $2.435214974-0.146383091-2.8324344917$ H $2.0083151317-3.6992359494-1.2645508288$ H $3.6459875508-4.2623592327-0.8972788568$ H $2.9681321237-4.5194845543-2.5107087305$ $\mathrm{H}-0.60553467962 .0687368273-5.3245921705$ H $-0.44334626890 .5875232291-4.3706869999$ H - $1.59783453940 .6576056554-5.7142979745$ H -3.4223770199-2.6073659372 0.0981836893 H - $4.6969475285-2.81933510361 .3068507211$ H $-4.0618061299-4.22400294780 .4395566614$ H - $1.0120212328-3.53584804660 .627237814$ H - $0.741307282-4.34466633722 .1755077665$ H - $1.7519548474-5.11270089330 .9434428382$ H $3.1430938951-0.9427778157-3.6292023453$ H $1.7059578283-1.7246002646-2.956606805$ H $2.717172915-2.5876524402-4.1249730848$ H - $2.73134792933 .4410811931-5.2435322216$ H $-4.07945030972 .9393922228-4.2064235416$ H -3.6287606743 $1.9654479139-5.6077267322$ H $-4.245608053-4.05927798593 .4659793208$ H -2.6278432414 -4.7019835313.8054337039 H - $3.5983949507-5.38894241642 .501693676$ H $5.4543590418-1.8619579557-3.136668646$ H $5.6606224189-3.1997161493-1.990752541$ H $4.8847347972-3.4800911753-3.5514185381$

${ }^{2} 7_{\mathrm{DFT}}$

$\mathrm{E}^{\mathrm{0}}=-1964.875663 ; \mathrm{G}^{0}=-1964.119455$

$\mathrm{E}^{0}{ }_{\mathrm{Et} 2 \mathrm{O}}=-1965.36242 ; \mathrm{G}^{0}{ }_{\mathrm{Et} 2 \mathrm{O}}=-1964.606212 ;$ Charge $=0 ;$ Multiplicity $=2$ 


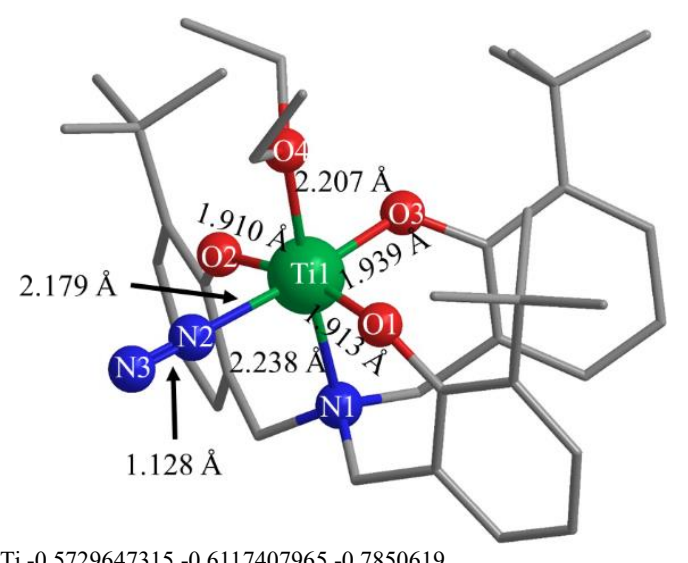

Ti - $0.5729647315-0.6117407965-0.7850619$ O - $1.8679946504-1.1300402373-2.1325512839$ O $-0.1304156441 .0969696273-1.5216396627$ O $-0.5597838158-2.34358453970 .0200938598$ N 1.49386593220 .15898530251 .6786959105 N $0.7932732451-0.09529575120 .8318650054$ N $1.0972630051-1.3586411001-2.0739405761$ C $-1.7554964265-0.8913049903-3.4461685623$ C $1.0395747626-3.6842977295-1.0643863565$ C $-2.8783531792-0.5003648998-4.2244121015$ C $2.1169152118-0.2898020676-2.279628234$ C $0.54925876911 .6304261365-2.5496049504$ C - $0.4959410827-1.0687976302-4.067672851$ C $1.8278244267-2.4567120793-1.3671303865$ C $1.66785603940 .9183750028-3.0231691862$ C - $0.1381873546-3.5753232273-0.2960232878$ C $2.37948313381 .3680404065-4.1298295154$ C $-2.6381895577-0.1161659697-5.5467965082$ C $-0.3122219875-0.673162359-5.390422656$ C $0.570484002-1.8670228548-3.3757248793$ C $-1.370471391-0.1549976792-6.1225355238$ C $1.5066239746-4.9333130567-1.4681536006$ C $-4.3024018892-0.5722403232-3.660957222$ C $-0.828451091-4.74627131240 .112574732$ C $0.17361266782 .8505113282-3.1634513292$ C $-0.3138390691-5.974413826-0.3095019876$ C $0.91927345863 .2621550001-4.2728254475$ C $-2.0809060599-4.6656399830 .981768285$ C $2.00088179062 .5402824436-4.7678694203$ C $-5.3569699695-0.3221369226-4.7415317099$ C $-4.558718171-1.9784470508-3.0944870253$ C $-4.51900611470 .4725688879-2.5602168977$ C $-3.176797099-3.91084008210 .2171746012$ C $0.8303931677-6.0845421453-1.0952319914$ C $-0.983819113 .6921584447-2.6297844091$ C $-2.6368527378-6.04594474121 .3368721493$ C $-1.7383040425-3.96170531532 .3028497256$ C $-2.27600320172 .8721072703-2.6531692409$ C $-0.66864748274 .139782036-1.1955070758$ C - $1.21855791034 .9525047078-3.4642589861$ H $2.9783408735-0.7387487982-2.8011642813$ H $2.4625274943-0.0144436319-1.2728296367$ H $2.7068211619-2.7301325855-1.9734724433$ H $2.2079522424-2.0046727635-0.4405289751$ H $3.23090011880 .7886072272-4.4836259337$ H -3.4705499428 $0.2186176795-6.1580544006$ H $0.6721860668-0.7859952677-5.8420488792$ H $0.1650114456-2.8610756497-3.1480309782$ H $1.4234248034-2.0150758693-4.0585082613$ H - $1.2257773080 .172422809-7.147523861$ H $2.4122724935-4.9889569991-2.0696526617$ H - $0.8260493356-6.8852896605-0.0171549824$ H $0.6461156384 .1844211952-4.774830115$ H $2.5437388162 .8991397054-5.6368568045$ H -5.2876587899 $0.6872464122-5.1607834117$ H - $6.3560551824-0.4249744407-4.3048787883$ H -5.2790196901 -1.0389808376 -5.5657313461
H $-3.870589625-2.2209696745-2.2828406301$ H $-4.439939454-2.7383363378-3.8743064117$ H -5.5848459365 -2.0485514572 -2.7136368363 H -3.7163476196 $0.4410197832-1.8230588526$ H -5.474115506 $0.2938616993-2.049211005$ H - $4.55099199391 .4828127866-2.9801903002$ H -2.8161052867 -2.9496934352 -0.1513323271 H $-4.0518723467-3.7419103630 .8573039464$ H - $3.5061550635-4.4907754859-0.651561618$ H $1.18784703-7.0616809146-1.4047525225$ H - $2.9363221435-6.61149517840 .4485031306$ H - $3.5262948559-5.92746201211 .9643358291$ H - $1.9160917421-6.64912567191 .8989838721$ H - $1.0198920406-4.55675977992 .8767331586$ H - $2.6386567635-3.84380187012 .9180635587$ H - $1.297067661-2.97693386792 .1370093511$ H - $2.49817460392 .5192789938-3.6668846854$ H - $3.12372262913 .4833102611-2.3158523383$ H - $2.20651237731 .9927749512-2.0107485899$ H $-0.47281678383 .2834448807-0.5461348483$ H - $1.50998626744 .7060960662-0.7773418345$ H $0.21432726514 .7872362721-1.1736346687$ H - $0.34501929785 .6127827619-3.4737031584$ H - $2.0528009875 .5188334095-3.0374992645$ H - $1.47854514424 .7151871486-4.5010462244$ O - 2.21499901960 .31280911550 .3635756521 C -1.93812217171 .4686371061 .183823268$ C -3.00697338782 .52842298961 .0899003594$ C $-3.4945110201-0.31573618160 .6005490948$ C -3.6338603987-0.8412352072 2.0054080303 H -0.98786469271 .8449994340 .7962584829$ H - 1.77375650551 .13981626212 .2193260415 H - 2.65198799123 .44132279221 .5749804655 H - 3.93776549992 .23497027861 .5834592384 H -3.2251809302 2.76549161130.0438956163 H -4.28370674370 .40490051960 .3561093393$ H - $3.5429247888-1.1092964527-0.1495527255$ H -3.6804348237 - 0.03678838542 .7460390758 H -2.8016682468 -1.5014987914 2.2614384537 H - $4.5585916692-1.41820350482 .0908191334$

${ }^{2} 8_{\mathrm{DFT}}$

$\mathrm{E}^{0}=-1731.215383 ; \mathrm{G}^{\mathbf{0}}=-1730.597671 ;$

$\mathrm{E}^{0} \mathrm{Et2O}=-1731.640474 ; \mathrm{G}^{0} \mathrm{E} 2 \mathrm{O}=-1731.022762 ;$ Charge $=0 ;$ Multiplicity $=2$;

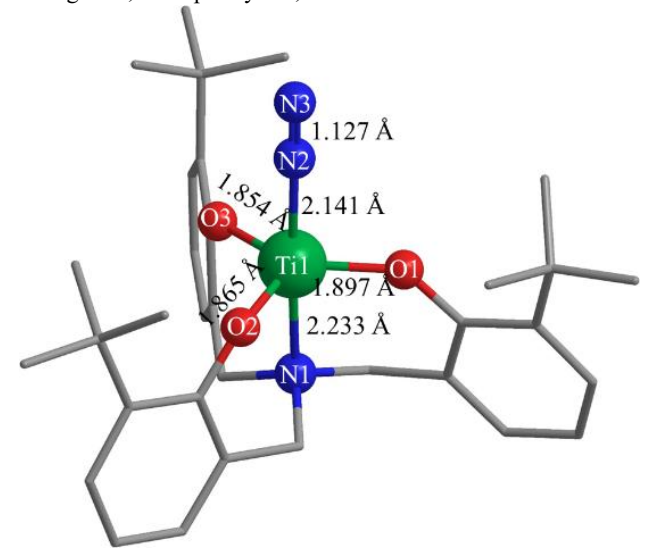

Ti 2.75195169821 .86402109551 .5729334175 O 4.49590424111 .11949270941 .6271923022 O 2.65203170273 .25281041720 .3324148943 O 1.5915160371 .32046350952 .9127293054 C 1.1159082391 .88367952236 .9695126368 N 3.44215273633 .39202238983 .0475208876 C 0.76970229590 .66334062366 .3939746926 C 1.62920573242 .89959226316 .1745760326 C 5.7680261272 .46155312923 .113845391 C 8.15104292412 .64719089832 .8075292955 C 2.95046286985 .2847786821 .4791780921 C 1.7284228547 .24859671140 .8088204525 
C 8.06193009251 .80933564591 .6973417612 C 3.96628079464 .59632226152 .3344694296 C 5.68959976411 .61049056631 .9922349311 C 1.7768614882 .70369840614 .8037469981 C 1.41620518631 .46661890414 .2389456374 C 2.29364155313 .79275781143 .9158321257 C 0.91245498470 .40927602045 .0262981657 C $1.10606506016 .5091064775-0.1942124933$ C 2.31724689484 .55320051530 .4557669091 C 4.52451948132 .78130305623 .8797941569 C 7.00074866152 .97809089813 .5086344994 C 2.64407849356 .63228374841 .6510711523 C - $0.23129503673 .2634462414-0.7917416166$ C $0.63848124494 .3338863031-1.4705994133$ C $6.168390085-0.98963843990 .3680862935$ C $1.36752031555 .1508034453-0.4011799243$ C $0.5685707388-0.94659346314 .4060225544$ C 6.76269033640 .38185308150 .0119193893 C $1.63544032693 .6663027919-2.4301882076$ C $-0.5029238454-0.78643176013 .3167533353$ C 6.85011320681 .27423817411 .2530646422 C $1.8331977127-1.5831802783 .8064498575$ C $5.89667039851 .05932769-1.0626000453$ C $-0.29076711355 .2105608968-2.3132758329$ C $0.0180523429-1.91927955335 .4512167353$ C $8.14259852960 .1367272422-0.6033532272$ H 0.99265742632 .03250354978 .0377926451 H $0.3820750478-0.11890812497 .0383147595$ H 1.91760523983 .85470799626 .609373637 H 9.1148756636 3.0424579868 3.1133314841 H 1.48947819628 .30028826730 .9332632974 H 8.97370927521 .57227823361 .158736905 H 4.81258168984 .24866586411 .7291617947 H 4.3697820765 .29756843343 .0829683329 H 1.50934456864 .15440436113 .2360371883 H 2.6024153047 4.6549683078 4.5293944809 H $0.38698954087 .0114473056-0.8329657918$ H 4.09130149221 .87562585664 .3252963418 H 4.75943531393 .46615906684 .710805922 H 7.0468475401 3.6387202046 4.3728561536 H 3.13407996817 .19193196732 .445693531 H $-0.98940773273 .725456267-0.1505205799$ H $-0.75190988132 .6631062322-1.5465171655$ H $0.35982440162 .5792419822-0.1772873324$ H $6.7803251025-1.49494464861 .1228204631$ H $6.1462656383-1.6273303263-0.5231355324$ H $5.1511843302-0.90590077840 .7542183238$ H $2.26150302014 .4163420642-2.9245973462$ H $2.29285464882 .9611265142-1.9192438184$ H $1.09049385073 .1220930665-3.2097058155$ H - $1.4210907995-0.35913609553 .7333533908$ H - $0.7536381674-1.76613449112 .8946585325$ H - $0.1689380789-0.14213548762 .5018330909$ H $2.2670633985-0.97513330163 .0094982702$ H $2.5993333473-1.72813867064 .5754359019$ H $1.5938557476-2.56627276473 .3856553953$ H $6.31264972122 .0324452855-1.3449224656$ H $4.87024973851 .2157739738-0.7244969386$ H $5.86303459260 .4369096097-1.9641728554$ H $0.25586209736 .0025506155-2.8359434368$ H - $1.07701863955 .6786881562-1.711915475$ H $-0.78325941564 .5942524658-3.0717546732$ H - $0.8993035438-1.54524043085 .917944264$ H $0.7438414575-2.127667496 .2442542187$ H - $0.2230394124-2.87193685754 .9696283094$ H $8.61948344751 .0656654037-0.9336078326$ H $8.820961076-0.36506355840 .0948127582$ H $8.0392466096-0.5083242609-1.4815288042$ N 2.02515679920 .37339695410 .2183651202 N $1.6444691487-0.3995153387-0.5079338621$

${ }^{4} \mathbf{9}_{\mathrm{DFT}}$

$\mathrm{E}^{0}=-2255.222153 ; \mathrm{G}^{0}=-2254.348861 ;$

$\mathrm{E}^{0}{ }_{\mathrm{Et} 2 \mathrm{O}}=-3398.975249 ; \mathrm{G}^{\mathrm{o}} \mathrm{Et2O}=-3398.101958 ;$ Charge $=0 ;$ Multiplicity $=4$

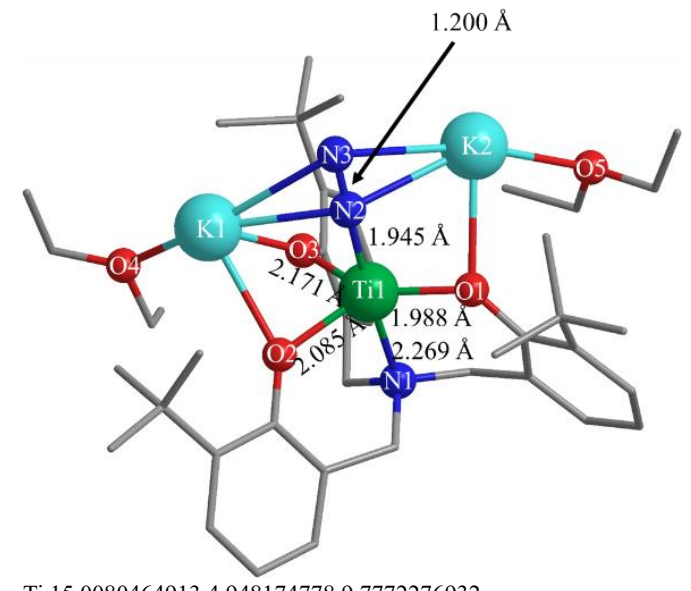

Ti 15.00804649134 .9481747789 .7772276932 K 15.5487183374 8.15322525749.3580497631 K 12.09496679764 .39879736828 .0846573899 O 14.24096523146 .585004100310 .9799391497 O 16.82687081215 .86516624510 .2210665155 O 14.26414218763 .19970722539 .1928798136 O 15.88551291239 .480627654611 .6915181709 N 15.39988424773 .856193268211 .7271660853 N 14.48356978585 .87272943418 .147850945 N 14.02783870256 .49733814837 .2304833955 O 10.71650328222 .43802805779 .3694295955 C 15.36236183224 .829156736512 .8508147766 C 16.74826817563 .253188831611 .6015377264 C 13.51997108186 .354311570412 .0574828919 C 14.01899149955 .462608144113 .0504496085 C 13.28683613395 .198756874514 .2051357518 C 17.95249271695 .351260208710 .6882482922 C 17.9415292664 .165460287511 .4792747404 C 12.24213933256 .967602586412 .2725983319 C 14.4012682922 .78177006611 .9788169512 C 14.49057758340 .335167329511 .4215245233 C 11.55475463496 .672181122613 .4524197828 C 14.43348041321 .657360933510 .9872029899 C 14.43083334211 .94584796099 .602007208 C $14.5595975855-0.706864806210 .5051305797$ C 19.11950395143 .704771639412 .0679527358 C 12.05383413415 .801533529714 .420153397 C 11.65323385327 .940107249711 .2450526394 C 20.33254737244 .343241276211 .8610118978 C 19.21619585155 .974608630610 .4256174846 C 16.139542623910 .840210644811 .9891567056 C 20.36347947875 .455390436511 .0249653557 C 19.30430191657 .20324137779 .516072725 C 14.63699678720 .9004235758 .6616188083 C $14.6635237405-0.41091597029 .1472080309$ C 11.5666853857 .28544086599 .8566426646 C 12.53134360889 .197579915811 .1697079814 C 15.224732868 .713750470713 .9397390641 C 16.17271205428 .582768567512 .7694933305 C 10.23732777638 .391900440711 .6092801383 C 18.63356634596 .93389113978 .1572275966 C 18.64737860428 .390500585910 .2355858275 C 15.942467756511 .640346604210 .7259406536 C 13.91737144972 .14016202146 .5334384762 C 14.95870571241 .22126519387 .1975013345 C 20.74794548217 .60890297799 .2064846587 C 16.32349575411 .92928280267 .1493848272 C $15.0486861593-0.05065585576 .3508340577$ C 10.95086170393 .877990095211 .2681400679 C 10.89767289432 .45260532510 .7875247191 C 10.76076606121 .10884974518 .857623908 C 10.19986042061 .10089192937 .4584328648 H 16.12619940945 .579325660812 .6145072374 H 15.67265363054 .312678117413 .7766648236 H 16.70318543942 .592688155910 .7226390353 H 16.91226505232 .580946294512 .4643154525 
H 13.70181863284 .51255503814 .9437388653 H 13.4240219143 .2787075812 .0031641003 H 14.57025843282 .371431258312 .9918412909 H 14.48732279960 .131038192912 .4915003835 H 10.58545851947 .129668862313 .6290377746 H $14.5836987677-1.738836913910 .8417777345$ H 19.07324460172 .808965635612 .686865167 H 11.48536347385 .601454655315 .3235054435 H 21.24224837423 .975324493612 .3255614916 H 17.169918294610 .949140357312 .370925602 H 15.461316152711 .197826908612 .7800052327 H 21.3213724825 .930668212410 .8357390771 H $14.7877266234-1.23480394818 .451243458$ H 12.54148318536 .91180721299 .533636018 H 11.2001287207 8.00640955199.1148361349 H 10.85122121736 .45232400219 .8866416486 H 12.45876705859 .774404251812 .0994788008 H 12.21079658579 .852201703610 .3480615287 H 13.58424194158 .94418397111 .0424011541 H 14.18648197388 .59211889713 .6162039092 H 15.32481841449 .675524454514 .4528079668 H 15.43123896057 .927376813714 .6714857571 H 17.21895838758 .732445031513 .0850534381 H 16.09523579787 .587429434112 .31649281 H 9.54560651117 .544924572911 .6837724336 H 9.85589730259 .065408552810 .8339802419 H 10.20730682068 .936169949212 .5584867256 H 19.20081526036 .18079503637 .600818211 H 18.62708251977 .84800511937 .5476293557 H 17.6155776686 .55107922158 .2528036448 H 17.69915502258 .106357915710 .6885944794 H 18.49233499059 .24302634549 .5564644213 H 19.28461737568 .742852755811 .0543398576 H 16.645146921211 .32319709799 .9477795369 H 16.105691130512 .705338567510 .9056643708 H 14.922186967611 .518368582210 .3472031557 H 12.89573636981 .82928336886 .7889340458 H 14.01161166352 .08968851665 .4426375173 H 14.07762433633 .17827045426 .8305031266 H 21.29830366377 .906138886710 .1047300054 H 20.74848782568 .46750546758 .5254493867 Н 21.3026431284 6.7995973017 8.7207051536 H 16.3079250852 .86260103077 .7269470284 H 16.59103732852 .18140533176 .1163970232 H 17.11122376711 .28659139397 .5557430053 H $15.8356048518-0.72528611656 .7007741467$ H 15.28348332710 .21261761115 .3146712467 H $14.1032047315-0.60634380076 .34841542$ H 11.82896546494 .410173111210 .8743398253 H 11.04132166243 .925674140612 .3576326293 H 10.0478368834 .431616336310 .98671015 H 11.82392367891 .912467154511 .0415723012 H 10.06259180131 .904957098511 .2543926675 H 10.16367854770 .45084096879 .5088887092 H 11.79925057860 .73458005778 .8857488877 H 10.75728937921 .77037373046 .7938975446 H 9.15276139211 .41485744997 .4521880665 H 10.26177078490 .09925730817 .0260697197

${ }^{2} 9_{\text {DFT }}$

$\mathrm{E}^{0}=-2255.229964 ; \mathrm{G}^{0}=-2254.355175$

$\mathrm{E}^{0} \mathrm{Et}_{2 \mathrm{O}}=-3398.985408 ; \mathrm{G}^{0}{ }_{\mathrm{Et} 2 \mathrm{O}}=-3398.110619$; Charge $=0 ;$ Multiplicity $=2$;

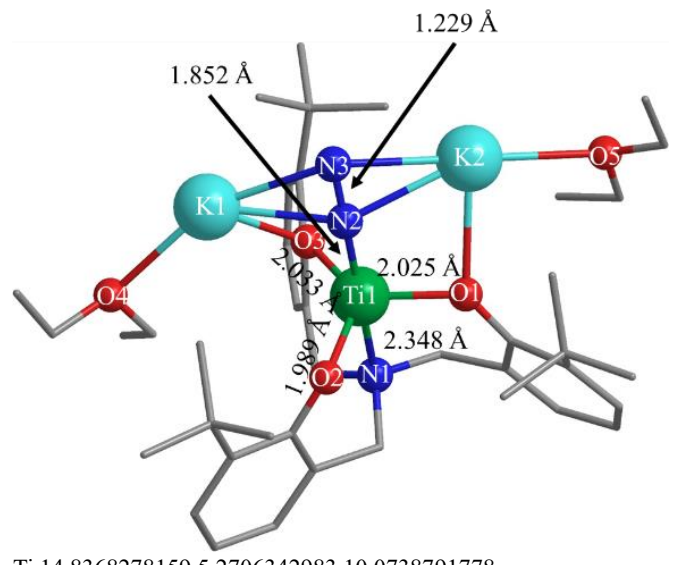

Ti 14.83682781595 .270634298310 .0738791778 K 14.70634157658 .70342944459 .6745304401 K 12.09392472134 .61995869028 .0746301418 O 13.79562345686 .46537065511 .3475781997 O 16.78802997035 .28778998129 .6860891874 O 14.08141350993 .48432428029 .4909023346 O 16.43872204389 .511075580211 .6114233512 N 15.37576047494 .168088482412 .0758753366 N 14.12489140376 .26740634528 .684168147 N 13.46472955086 .99550481477 .9461979501 O 10.70204542822 .24026183568 .268362084 C 15.42779441695 .089170657513 .2328781889 C 16.72144946473 .557022209911 .9068936218 C 13.35116555366 .375803847312 .5988155709 C 14.13027089695 .73981994613 .5936731819 C 13.70661643965 .734596161214 .9213237116 C 17.79073640595 .381135860310 .5478340114 C 17.8051258414 .57541879911 .71416154 C 12.07982860656 .912511940412 .9510711047 C 14.31492881273 .145783937112 .2974407053 C 14.48753038270 .696836911211 .799686151 C 11.71058325846 .902638246314 .2983496817 C 14.36744913122 .003725281611 .3315946642 C 14.35738313352 .26310349659 .9397388625 C $14.629605928-0.360624778310 .911532091$ C 18.82153692524 .72719148412 .6554379122 C 12.51581142246 .344777939915 .2886619098 C 11.10939925977 .386003793411 .8645366107 C 19.83890107775 .651583325312 .4567754219 C 18.86344615746 .286862196810 .3044334815 C 17.663544771310 .232848467811 .6584928269 C 19.85351072246 .405757068211 .2830044743 C 18.93856030657 .04980139788 .9779966529 C 14.66006124681 .20820815779 .0323820388 C $14.7538020745-0.08713057759 .5501955846$ C 10.7441097096 .169399217410 .9977070334 C 11.7151957978 .469266797710 .9509506788 C 16.16490462528 .708718758813 .9204471593 C 16.441360768 .365797479412 .4745523658 C 9.81518050037 .946918731512 .4575063736 C 19.0949635586 .04431540627 .8271532045 C 17.67154501567 .88665672938 .7359935731 C 17.637126054911 .299511061910 .5946775952 C 13.99537774422 .39670692146 .853863677 C 15.02863228031 .51064450787 .5752588803 C 20.1303935438 .00854604778 .9377370456 C 16.37598854492 .25237671637 .5776620243 C 15.18621480390 .22725204476 .7555585348 C 10.99762963942 .449668069910 .6324186056 C 10.93481453921 .49725062259 .4686829966 C 10.45034406921 .37762099747 .1692704282 C 10.27273875712 .20619614475 .9229236837 H 16.19767386265 .832153782812 .9911294286 H 15.80729419814 .538173320614 .1148749098 H 16.66359289952 .89355481811 .0351690549 H 16.95213749362 .926318272412 .7848406384 
H 14.33001537445 .243835917715 .6682701937 H 13.3575277963 .688696078412 .2434996303 H 14.39287319642 .752333104113 .327018121 H 14.48033632070 .519817260912 .8746607895 H 10.75824079517 .332171979314 .5934908002 H $14.7013062248-1.382373068611 .2720947992$ H 18.80488705154 .107620052613 .55185654 H 12.1954865286 .35801692416 .3260675216 H 20.6265698878 5.7747451415 13.1942031032 H 18.49875411789 .528763303611 .4939450806 H 17.806386434310 .677626961112 .655338943 H 20.672572407 7.103272916 11.1307069785 H $14.9477848732-0.91684590558 .8774320596$ H 11.6608775225 .714205679510 .6063967706 H 10.10345991866 .467534903810 .1560778348 H 10.20805334985 .417442598811 .5862803268 H 12.35436812049 .157149168611 .5203096828 H 10.91952428629 .064706683510 .4878637111 H 12.28816917748 .007508957610 .1436348486 H 15.1822383469 .177907925614 .0302635114 H 16.92120156199 .381662250414 .3354056176 H 16.16254290917 .798472619114 .5273811907 H 17.39829351667 .829591424412 .356609947 H 15.65248627527 .706646145812 .0902351634 H 9.2893318459 7.2107723746 13.0732873859 H 9.13874006928 .241596342911 .6484657283 H 9.99897323038 .833344203713 .0742994831 H 20.02057818385 .46973780547 .9372983708 H 19.13680237646 .5688515876 .8647376134 H 18.2534822576 5.3480137265 7.8037999358 H 16.80850160187 .24173401098 .5473093537 H 17.81585773188 .54375771757 .868100289 H 17.4708438858 .51929728819 .6097337443 H 17.539923300210 .85668401149 .5974199794 H 18.562418511511 .880353326510 .6088826796 H 16.803587555411 .990293542810 .7514048847 H 12.97477400442 .14853073037 .1648105991 H 14.06234897122 .25111873795 .768672135 H 14.1907595483 .45190873267 .0598104696 H 20.06737977248 .77851849969 .7173014425 H 20.15611979648 .52104795427 .9702326261 H 21.0853957171 7.4871799484 9.0576923711 H 16.3146147616 3.186234713 8.1469466039 H 16.68010780112 .50268959876 .5537243209 H 17.16294812081 .63279129848 .0210127922 H $15.9799864793-0.41776864747 .1435945944$ H 15.45151987710 .48117527065 .7243308223 H $14.2589066358-0.35792359346 .7280854945$ H 11.83588175053 .147197351910 .5283998511 H 11.16088635621 .898631904311 .5619476558 H 10.06703058023 .017520871710 .7268669705 H 11.87413008420 .9278677079 .369660534 H 10.11652679760 .7703397889 .5985889515 H 9.54983048890 .7760089047 .3761519175 H 11.28907503860 .6684678437 .0533308116 H 11.19558738982 .73436063395 .6596767982 H 9.47275090552 .94113666426 .0531617903 H 10.00987100141 .57256750475 .0730185918

${ }^{2} 10_{\text {DFT }}$

$\mathrm{E}^{\mathbf{0}}=-2038.188418 ; \mathrm{G}^{\mathbf{0}}=-2037.382768 ;$

$\mathrm{E}^{0} \mathrm{Et}_{2 \mathrm{O}}=-2610.295288 ; \mathrm{G}^{\mathrm{o}} \mathrm{Et2O}=-2609.489638 ;$ Charge $=0 ;$ Multiplicity $=2$;

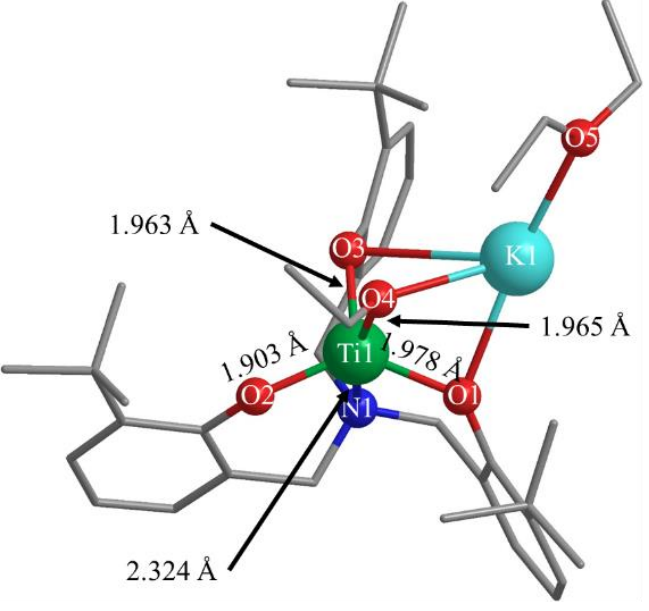

Ti 2.70426288411 .97635329631 .2043451701 O 4.49314028641 .17605842411 .3108566303 O 2.57804677483 .60581459440 .0893824074 O 1.6087628421 .2685878232 .5899694315 C 1.22034963941 .686697856 .6844684877 N 3.39747382263 .50176510472 .815407241 C 0.93562999990 .46951141316 .0692557016 C 1.65725490852 .7539617775 .9128142497 C 5.7342491952 .56033585072 .7998181727 C 8.13522468762 .68800712582 .5516684738 C 2.86357825565 .51917449481 .4415147859 C 1.57532142337 .50801467221 .0015422308 C 8.07738376381 .67653680781 .5944391433 C 3.8958918014 .7660413682 .2160058428 C 5.67037552421 .61266464651 .7491662848 C 1.78561658812 .62110501484 .5315343428 C 1.47996364071 .38819025623 .9144799816 C 2.23651900653 .78682619013 .7042596233 C 1.0618189890 .277866615814 .691289467 C $0.89449856496 .8298552021-0.0074990984$ C 2.20479371784 .85488786620 .3869967521 C 4.49888814272 .87489020743 .5880705524 C 6.96378148123 .09973852623 .1730437441 C 2.54234865516 .83993551241 .7417735051 C $-0.55197450983 .7152748174-0.5240429604$ C $0.29719891524 .7163383951-1.3238009825$ C $6.2515849703-1.318812081 .0674460158$ C $1.16667960925 .4968721191-0.3322442953$ C $0.7962023883-1.07919841034 .0340026711$ C $6.8415595022-0.13888166330 .2783392897$ C $1.11819475773 .9287746289-2.3630262269$ C $-0.3221959528-0.96538338682 .9865614586$ C 6.87209081621 .0967526911 .1877441888 C $2.0834566775-1.59657406363 .3705010861$ C $5.98880187770 .0681604788-0.9809478847$ C $-0.65226705855 .6394186701-2.0916286168$ C $0.3570455301-2.12852566535 .0578862764$ C $8.2452850628-0.5334084389-0.1869531649$ O $2.34395090110 .9238272743-0.415025273$ C $1.10250105380 .3470775044-0.6997197281$ C $1.1685772015-1.1644293626-0.5890981992$ H 1.11337403931 .7913402227 .7600326957 H $0.6124417351-0.3586769076 .6921813707$ H 1.90438842653 .70837772056 .3756535894 H 9.09171958033 .11731226852 .8341980865 H 1.3294682418 .54253648521 .2211997805 H 9.01001330911 .3209285471 .1668811672 H 4.7434055744 .4822767431 .5769186569 H 4.30429000635 .40743111153 .0179300272 H 1.42934108484 .14042048533 .0463637132 H 2.48988440294 .62840534014 .3742015111 H $0.11701094617 .3609413573-0.5476724757$ H 4.08995089591 .95024308354 .0161840206 H 4.76188051783 .52903337744 .4387076916 H 6.99198766183 .83962756483 .9721899641 
H 3.0577419037 .33831115782 .5614395568 H - 1.22091267564 .23517346840 .1689039045 H - $1.16538829623 .1004843254-1.1940153541$ H 0.08146758963 .03705511830 .0572956106 H $6.8541650699-1.53110162671 .9562486568$ H $6.2376693576-2.22270237470 .4465783709$ H $5.2281434131-1.10453962461 .3842334361$ H $1.93377108594 .5361733838-2.7775446932$ H $1.5226317553 .0158281595-1.9206356645$ H $0.47325924793 .6321604524-3.1987129775$ H - $1.2569815879-0.6348526333 .452463117$ H $-0.5065442266-1.94421003692 .5268741522$ H $-0.0652888315-0.25864189462 .1956970405$ H $2.8819821524-1.71541403384 .1114849504$ H $1.9037910731-2.57815698552 .9147962751$ H $2.442869235-0.92067913842 .5915122545$ H $6.33654400980 .9420440436-1.549723627$ H $4.92894713690 .1673301037-0.7274090609$ H $6.089262472-0.7965246748-1.6495661588$ H - $0.10981182346 .3949038592-2.6708155996$ H - $1.34913665966 .159281572-1.4276930768$ H - $1.25277315685 .049494908-2.7913390935$ H $-0.5718502444-1.84566554155 .5648496083$ H $1.1214316196-2.30499282145 .8222189308$ H $0.1766223657-3.08059579444 .5481479225$ H $8.72457280840 .2602163889-0.772699451$ H $8.9046532591-0.77557225580 .6521491687$ H $8.183826301-1.423352842-0.8225030764$ H $0.76158793950 .6321724368-1.7170601202$ H $0.31676776840 .7207142984-0.0132676671$ H $1.9171218295-1.5712300535-1.2786025438$ H $0.2026290331-1.6317632515-0.8108788541$ H $1.4649073364-1.45314770050 .4247746965$ K $4.09706414922 .46443042-1.7212898295$ O $4.99903444351 .2982698482-3.9855797207$ C $2.91226732750 .1739288835-3.7343892296$ C $4.24321068750 .17664732-4.4406680498$ C $6.1914226331 .5151625315-4.7358050547$ C $7.23148246580 .4261330841-4.5832206163$ H $2.3003712406-0.6660435977-4.0715288288$ H $2.35081827551 .0922110558-3.941734832$ H $3.0253953050 .0726885601-2.6484875726$ H $4.11832623240 .2484748218-5.5339913387$ H $4.7891779208-0.7561990248-4.235490345$ H $5.92758741531 .6510051452-5.7975601648$ H $6.58593095092 .4722195939-4.3762351239$ H $6.8922142014-0.5277089227-4.9956918461$ H $7.48996029620 .268413094-3.5323494002$ H $8.14269243910 .7047167048-5.1189282587$

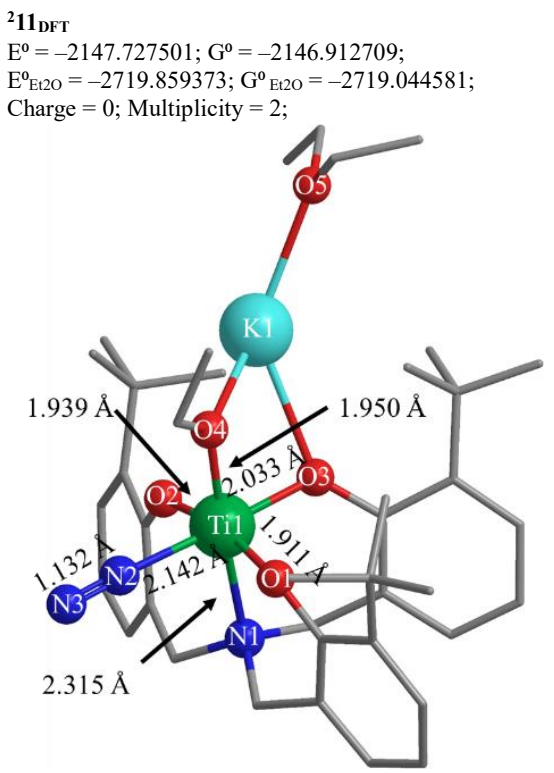

Ti $1.0092047826-0.69286721241 .3147549813$ K -2.108010158 - $1.1814888195-0.2134974703$ O $0.4363787619-0.1643858468-0.562745263$ O 1.55314803581 .03188572631 .9308058378 O $0.935727715-2.53693607340 .7213222464$ O $-0.8527806266-0.50387449211 .8622807801$ N $1.9880766418-1.75193057354 .252673038$ N $1.6228174796-1.40307639393 .2399921358$ N $3.1952280457-0.83850315040 .5679836655$ C $1.10038688710 .7782996275-1.2385171246$ C $3.0771822225-3.34713800150 .1328606377$ C $0.4153900551 .8394749968-1.8968561595$ C 4.09966161380 .06625600791 .3245458703 C 2.51135133581 .94981019141 .7821946956 C $2.51254424390 .7015353637-1.3309940362$ C $3.729117772-2.20612920180 .8405473707$ C 3.78625051931 .52471517081 .3595636879 C $1.6704107624-3.46181656720 .1125290866$ C 4.7894595342 .45545176671 .1094203806 C $1.18149923032 .8514885031-2.4793467297$ C $3.22823815191 .7308077444-1.9377031686$ C $3.2181432853-0.5488698147-0.8907418811$ C $2.57405789722 .826917558-2.4803903719$ C $3.8698699104-4.3302440833-0.4561056213$ C - $1.10838573691 .8068432463-2.0473953901$ C $1.0676417579-4.5445290983-0.5868382824$ C 2.27062693893 .32225602072 .046660867 C $1.9041535617-5.519224791-1.1327580749$ C 3.30752599354 .21914313991 .7749457137 C $-0.4308071494-4.5356691917-0.8858739114$ C 4.54857307353 .8093107741 .2949969626 C - $1.64281884072 .9911977888-2.856159504$ C $-1.48805323010 .5343759466-2.8328061544$ C $-1.82987385151 .8453483155-0.6880492052$ C $-0.654846759-3.3609692891-1.8561605129$ C $3.2922889409-5.437124632-1.0603467123$ C 0.94766382423 .78764732652 .6547898993 C $-0.9023129028-5.8083315368-1.5926326059$ C - $1.2917319903-4.38961185380 .3790231963$ C -0.22288068093 .39439377441 .7492573337$ C $-1.2911735043-0.14662270923 .1403552077$ C 0.77413139513 .14389753424 .0388536084 C 0.90095883845 .30559461062 .8389985438 O $-4.6315864803-0.5745879091-1.0231971277$ C -2.65214672530 .52099173313 .066974539$ C $-4.4736722919-2.4092755658-2.5271202377$ C $-5.3213253111-1.2516482134-2.066796867$ C $-5.408428840 .451879333-0.4043874735$ C $-5.73816199031 .6073142166-1.3236552483$ H - 1.33968795941 .23812682410 .0767936297 H - $0.2174794359-2.4303580228-1.4753862653$ H $5.1264798069-0.07311656190 .9417105704$ H $4.0954548021-0.32288870382 .3535751125$ H $4.8067632741-2.2144507860 .6005914874$ H $3.6544390206-2.33626561471 .9284528911$ H 5.76290992432 .10424725490 .7693313036 H $0.6803482843 .6875862134-2.9584487464$ H $4.31486701411 .6634126598-1.972826984$ H $2.7429405934-1.4160227106-1.3681913908$ H $4.2676387203-0.5222665899-1.2334327537$ H $3.13518710143 .6404887879-2.9296069853$ H $4.9526004707-4.2196278605-0.4250690228$ H $1.4635329718-6.3650133789-1.6515772036$ H 3.14685964835 .27868597831 .9467186817 H 5.32302412814 .54248328451 .090281001 H - $1.42707464633 .94668413-2.3668687072$ H - $2.73219364152 .9096887927-2.9510697328$ H - $1.22562735183 .0237485143-3.8676421043$ H - $1.17367095650 .624476583-3.8772826982$ H - $2.57811097640 .3822678098-2.8249978568$ H $-0.9909263009-0.3567122695-2.4381969508$ H - $2.87288527171 .5186905432-0.8059978862$ $\mathrm{H}-1.86221138992 .8661456431-0.2982304784$ H - $1.7239925509-3.2230272895-2.0755130198$ H - $0.1621936699-3.5641152669-2.8121240584$ 
Н $3.9099366758-6.2143584462-1.4995353684$ H - $0.4127243337-5.9522902242-2.5604099011$ H - $1.9805835405-5.7445072146-1.7796594506$ H $-0.7248947679-6.7016092607-0.9848581587$ H - $1.1798757427-5.26926179531 .0205395432$ H $-2.3554036834-4.3328682660 .1037593053$ H - $1.0163495601-3.5218472370 .9805544116$ H -0.06884611893 .77954385240 .7343465437$ H - 1.16137066083 .81511164442 .1344711022 H - 0.33260322012 .31068074491 .6840554999 H -0.57648319510 .54874931463 .6145861826$ H - $1.3436561019-1.03124646453 .8014568643$ H 0.8591156612 .0559623993 .9858755504 H -0.21179078293 .38959225994 .4537569302$ H 1.53376420213 .51094387844 .7374059118 H 1.69291061665 .66654342143 .503547099 H -0.05725832985 .58725978373 .2881567683$ H 0.98808244735 .837686661 .885478773 H -2.6044785369 1.41092710072 .427823788 H - 3.00867595470 .83006668424 .0548021534 H - $3.4020382964-0.16174308672 .6445360662$ H - $4.9765814899-2.967053006-3.3199368521$ H $-4.2731183842-3.1081388889-1.7072232157$ H - $3.5181574937-2.0577107677-2.9312110656$ H -6.300617177 - $1.5962936192-1.6951924378$ H $-5.5147368249-0.565274407-2.904893076$ H -6.3274480665 0.00720921980 .0105514601 H -4.80088067540 .79627469870 .4401912878$ H -6.4338621398 $1.3225626136-2.1173504674$ H - $4.83132408892 .0049790735-1.7904238137$ H -6.2057741838 2.415247974 - 0.7554574187

${ }^{4} 12$ DFT

$\mathrm{E}^{0}=-2671.717787 ; \mathrm{G}^{0}=-2670.648917$

$\mathrm{E}^{0}{ }_{\mathrm{Et} 2 \mathrm{O}}=-4387.176706 ; \mathrm{G}^{0} \mathrm{Et2O}=-4386.107836 ;$ Charge $=0 ;$ Multiplicity $=4$;

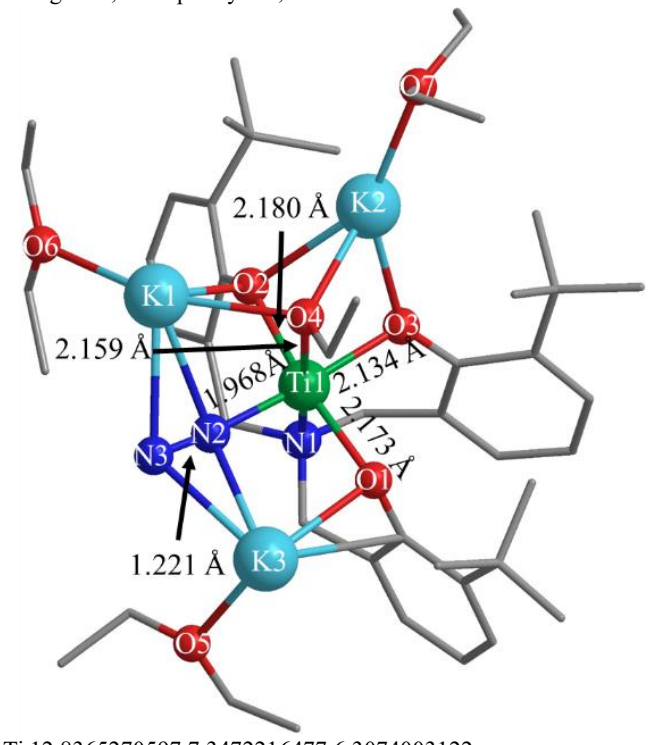

Ti 12.83652705977 .34722164776 .3074003122 K 13.35747636599.55622447769.1478639772 K 10.75620362095 .37378484227 .9490624775 K 10.36561049186 .61893473764 .0483235898 O 15.65180975348 .648712507910 .2050243038 O 12.78821887117 .52538330764 .181417054 O 13.4689783189 .41665712446 .5061519236 O 12.37250153265 .2878326195 .761477608 O 10.68925658657 .56597216276 .3604084134 N 12.58495087046 .92463910349 .4456359632 N 12.81258457887 .0718458818 .2555729168 N 15.0203622826 .85254963496 .0429285041 O 11.3584712212 .71821485888 .3677018481 C 13.6900578228 .26201661673 .5530436111 C 14.56083588284 .32436770226 .0269366591
C 13.32084102769 .24890305832 .589765071 C 16.86847912229 .34050354189 .9438980058 C 15.80661697957 .79202294356 .8753825306 C 14.6454931199 .99791849576 .3809688619 C 15.06900312618 .03538184763 .8067184168 C 15.19285757345 .52187829236 .6831715726 C 15.84761014749 .23636177596 .4864567183 C 13.2099428224 .29137098055 .5600797318 C 17.09396898599 .84130646046 .3435365433 C 16.36654110496 .963044336211 .8385360422 C 15.83305592197 .250557984610 .4525718247 C 14.3271708510 .04151314022 .0339484372 C 16.0350091138 .8416371123 .2123394559 C 15.46791664666 .84266554354 .6281713217 C 15.67288154269 .87033435152 .3526956477 C 15.35661945333 .17935351695 .9396599156 C 11.87536704389 .32947585872 .0889950181 C 12.79978709483 .12437967064 .8205206361 C 14.766985737511 .41506944996 .1882253467 C 13.62918836782 .00321340724 .7922121813 C 16.0442235511 .96824884856 .0586609112 C 11.57673049493 .19668211393 .9018038567 C 17.208176894611 .20649484686 .1218322372 C 16.551747272810 .78635370529 .6583546441 C 11.667043893310 .43979231011 .0565870006 C 11.56502752127 .99954115831 .373379823 C 10.85176553269 .58561484083 .2150842693 C 11.90531377294 .27546151922 .8493879235 C 14.89068317991 .9971585165 .3828198989 C 13.522622600112 .30360912826 .0924461516 C 11.31954938841 .89531440143 .1372599306 C 10.26854897783 .50307422874 .6503047445 C 13.42744756453 .60576216579 .1639861663 C 12.679962833911 .87022713724 .8900197303 C 10.24757133388 .78362035276 .842651249

C 12.660406732212 .18831968647 .3606672749 C 12.75428859692 .43225928428 .5080953572 C 13.871909371513 .78330227575 .9221143371 O 7.92946614856 .72020672642 .8455387068 C 8.82069399219 .09505455056 .4083338169 C 10.67534822441 .63005624477 .773308488 C 8.56068443895.49544835950.90463502 C 9.19375015441 .90996707797 .789980834 C 7.59951173836 .50433998791 .4787349469 C 6.90963425197 .39590524093 .576138876 C 6.68592616328 .82852510333 .1448430799 H 11.11682202299 .09096416934 .1531368966 H 12.30180544445 .19151469523 .3018904993 H 17.37154319988 .87848512569 .0765881157 H 17.54779543939 .252815606810 .8062045243 H 16.84946386497 .41999292086 .9373859405 H 15.37773378567 .69088085357 .8832633207 H 16.2780982526 5.3157160375 6.769381946 H 14.8216911825 .65881982867 .7079266105 H 17.98540566239 .21573957426 .4122298371 H 15.70217287017 .374425030512 .6034985993 H 17.3657455265 7.3799998898 11.9958542897 H 16.43494824715 .883157625611 .9978620668 H 16.50163140446 .83397468129 .680166986 H 14.84033152976 .80410770810 .3097932831 H 14.060430666810 .82076943291 .3243745547 H 17.08431111748 .66299946733 .445638454 H 15.02192286355 .93893476464 .1908788149 H 16.56546608076 .71508959334 .5961767367 H 16.426762456410 .51723551681 .9137807099 H 16.37360068943 .23107632446 .3286659469 H 13.29961580691 .11053685874 .2675747552 H 16.142883141213 .03745293375 .897036766 H 18.180746127811 .67547031476 .0052287503 H 15.951581127910 .89010826038 .7464660055 H 17.46972256111 .35270110049 .4870037437 H 16.025364757311 .24596697910 .5025575434 H 11.893070528111 .42645066181 .4752613495 H 10.619056159810 .45024127180 .7337319459 H 12.286211020310 .29840519710 .1649774182 
H 12.1469498467 .92086187560 .44950276 H 10.50016334497 .93770495741 .1025038331 H 11.83974295377 .13441392181 .9841818699 H 9.85056296759 .25346143332 .8965115848 H 10.770526674110 .65441389773 .4321580096 H 11.03244599594 .50821013062 .2209801839 H 12.68768787143 .90585803072 .1795141512 H 15.51634193081 .11067671725 .3465363685 H 12.16484131561 .61559223422 .5020304369 H 10.44617963862 .02015824122 .4858835803 H 11.1096999091 .05778129873 .8124795931 H 9.93461460732 .61943756675 .2031986633 H 9.46150404653 .75366006623 .9458859019 H 10.39577973234 .31653612365 .3666571348 H 13.33049847594 .51054021748 .5575860957 H 14.49859166053 .41240681539 .2739450667 H 13.0113494473 .814542811810 .1543737658 H 13.232219056812 .01503562583 .9551984788 H 11.750093485212 .45334048394 .8367019153 H 12.43000482410 .81147914764 .9702408409 H 10.89575771929 .61896765826 .5000128445 H 10.28581599398 .82750771067 .960631678 H 12.212310212611 .19333449727 .4242799416 H 11.842109974212 .91769432597 .3359531606 H 13.25555027312 .40028239038 .2614143814 H 13.18857920782 .23014703877 .5169502971 H 12.86371854621 .5160732359 .1131351256 H 14.453804118214 .17372181796 .765062644 H 12.94917120714 .36994312435 .8560720741 H 14.441660723213 .96534254775 .0056774731 H 8.76380245729 .14439187075 .312419784 H 8.462994677510 .05426917656 .8004068381 H 8.12655596758 .31302194446 .744233181 H 10.89599150470 .70587280558 .3344355326 H 11.05050224271 .47347160466 .7456676123 H $8.33372369735 .3022190584-0.1464238559$ H 8.50469700594 .54553136681 .4454482306 H 9.59196937585 .86029922090 .9547041332 H 8.95117798142 .81654963917 .224249876 H 8.83208681292 .0361888998 .8143782461 H 8.63819122891 .08722870717 .332967629 H 6.5596770956 .14310435881 .409823219 H 7.6505813642 7.4518355238 0.9189410736 H 5.97327244786 .81805696293 .5040285592 H 7.23744792377 .36044249324 .6217517925 H 6.28096816188 .89539487212 .1311933948 H 7.61915503379 .39920319563 .1781281043 H 5.97355806829 .31458213373 .817026669

${ }^{2} 12$ DFT

$\mathrm{E}^{0}=-2671.730202 ; \mathrm{G}^{0}=-2670.664665$

$\mathrm{E}^{0}{ }_{\mathrm{Et} 2 \mathrm{O}}=-4387.192462 ; \mathrm{G}^{0}{ }_{\mathrm{Et} 2 \mathrm{O}}=-4386.126925 ;$ Charge $=0 ;$ Multiplicity $=2$;

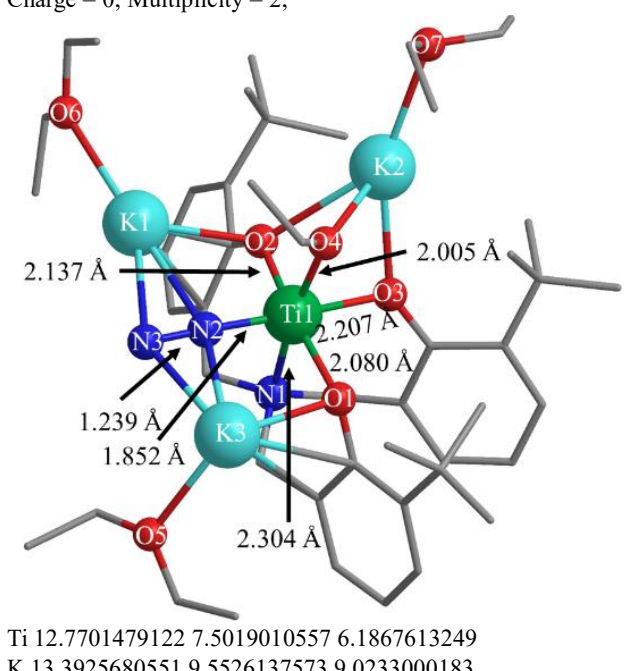

K 11.86869600024 .67279677398 .0661478913 K 10.53069164116 .68598941733 .8313293944 O 15.73364010529 .047902559910 .1836904908 O 12.89095437337 .63991109273 .9876034625 O 13.43501755829 .46605770596 .3478413893 O 12.55799954985 .46549779915 .5759525834 O 10.82731569337 .95511137035 .9887346258 N 12.86885539696 .89405865629 .2120454441 N 12.7705760937 .17123208518 .0088842358 N 15.01825082267 .02087073076 .0319385871 O 10.86397629472 .14725475058 .1383597295 C 13.81448832618 .37569094743 .4065720028 C 14.6931700364 .48124687465 .9682026214 C 13.50761569989 .31623036792 .3740219877 C 16.88380023019 .844587959 .9284859027 C 15.76786965757 .97741757146 .8858502246 C 14.573382224110 .11987772046 .284428128 C 15.17573879558 .1937190843 .7754264218 C 15.22767835165 .69462542436 .6743812261 C 15.79580565319 .41653877246 .4792285705 C 13.36154056544 .43211106695 .4521517853 C 17.019289718610 .07034276716 .3688440186 C 16.54458875697 .45599426411 .8612267559 C 16.02836424697 .675742706910 .4564762324 C 14.53685927710 .12133724881 .8826168766 C 16.16824482359 .00947887963 .2411483709 C 15.53119313227 .01475967834 .6330911609 C 15.854143901210 .00358534992 .3238565703 C 15.53049138693 .37107693415 .8633989215 C 12.11135709199 .33301341371 .7442596205 C 12.93543890123 .21776887654 .8074339964 C 14.627747443411 .53473462596 .0688626201 C 13.81044186992 .1312055184 .7597601334 C 15.882925950112 .14067324665 .9753771469 C 11.6172141993 .18634826414 .032059119 C 17.078054253811 .43305567756 .1042821965 C 16.428069750811 .23878957769 .5820229117 C 11.980837660310 .37071687270 .6271713834 C 11.87808631397 .95534875311 .0938798119 C 10.9953219459 .64304401482 .7604324506 C 11.80231791424 .12388839032 .8228117379 C 15.09941336852 .18304892435 .2860455227 C 13.340174442412 .34921864475 .9269002884 C 11.27664902921 .79831522443 .4830841571 C 10.41581484673 .59461315234 .9028823621 C 13.17617429831 .55917339788 .1361578267 C 12.536324384511 .84987531894 .7224940999 C 10.09499032358 .53014125597 .0133642174 C 12.467448645512 .20312302647 .185447857 C 11.78126001221 .11398620147 .7862692795 C 13.616327748213 .84106739865 .7351917182 O 7.9418691556 .55806254373 .0145801696 C 9.14133450027 .53797203257 .6588561683 C 9.51218541231 .74104419887 .9902434882 C 8.34966159816 .10613122040 .7179546984 C 8.6212392432 2.93137096438.2387753121 C 7.74809474087 .07508972661 .702949371 C 7.18465716457 .23839868974 .0148412346 C 7.67744905478 .63815876484 .3168248174 H 11.16218776259 .17944857613 .7362753028 H 12.29199601745 .06116599473 .109973157 H 17.45188635719 .40576934519 .0901212436 H 17.54527736599 .854251530610 .8094279547 H 16.81393563777 .62204352956 .9688105022 H 15.32739852257 .86333939247 .8880335817 H 16.31549368185 .54482193926 .8118879383 H 14.80662778285 .7910362627 .6877458403 H 17.93500796269 .4896850446 .491332313 H 15.82147575777 .811537235912 .6004416264 H 17.49307368137 .971927562912 .0392557765 H 16.71392485376 .390488148312 .0404930386 H 16.75312560687 .30910688729 .7097120665 H 15.08100904337 .145675802810 .285178364 H 14.313502332310 .8671217811 .1235881806 H 17.19904485888 .8644406393 .5641822266 
H 15.10865929586 .10935148934 .1782959665 H 16.62927304626 .88890951494 .6614598943 H 16.625034286210 .66035621041 .9315973298 H 16.54520788893 .45161410836 .2524181284 H 13.48712896681 .21241617414 .2776518941 H 15.939797306213 .20911032355 .788769021 H 18.031876174511 .94319476576 .0086481216 H 15.827750076311 .23952766198 .6643924138 H 17.285142659411 .88851718739 .3919608263 H 15.847330619911 .675364781410 .402082489 H 12.146442580511 .3872561991 .0002406735 H 10.96877851910 .3345535830 .2061192628 H $12.686638991810 .1898030847-0.1899297603$ H 12.5496740787 .81938588050 .2401761897 H 10.84687552277 .86482197230 .7202924465 H 12.09216503597 .13792762051 .7886190057 H 10.01890133529 .32321266042 .3614281577 H 10.920505223710 .72025613472 .934324859 H 10.84802105644 .32746277762 .3128093568 H 12.46089155953 .65218486232 .0875798557 H 15.75831915261 .32268962845 .2204824314 H 12.03099647961 .43187999862 .7812532873 H 10.32308430691 .83914176072 .9449042492 H 11.17116640911 .05720103664 .284935589 H 10.26861992462 .84987078675 .6928706862 H 9.49243005473 .62625272824 .3075388128 H 10.56117586054 .55956810365 .393332395 H 13.48025653112 .42646451817 .5398340731 H 13.89651409920 .77092739637 .9085831034 H 13.25678241571 .7968319829 .2017682092 H 13.10732801511 .96076462723 .7937760975 H 11.602052984412 .41922667254 .6228865753 H 12.292677277310 .79277646644 .840017836 H 9.51498988339 .39695866156 .6351822611 H 10.75148679548 .9447492257 .8057820663 H 12.090077545211 .18036015717 .2701274047 H 11.598871944212 .86995613277 .1298010885 H 13.03040088512 .47705197838 .089755928 H 11.69828364910 .90533814236 .7047762889 H 11.50912096040 .19127192218 .3239996992 H 14.163328493714 .27441846056 .58049002 H 12.667147695714 .37991662515 .6426820591 H 14.193438680514 .03418857094 .8254193619 H 8.48431423037 .08811550636 .9021986169 H 8.51049980447 .99754870828 .4284193007 H 9.71049040126 .7282243668 .1315866971 H 9.29432493570 .9224238728 .6955404158 H 9.35457637651 .33856767796 .9741495982 H $8.21832741686 .4667471355-0.3050187856$ H 7.8813083845 .122284270 .8026606757 H 9.42534193615 .98581402740 .8837122647 H 8.7928438533 .71506942487 .4924996847 H 8.7993528043 .35420032499 .2323699778 H 7.56842353782 .64664822918 .1814995563 H 6.66903033327 .20818709231 .5204074461 H 8.21549763048 .07052851061 .6042807194 H 6.12475984987 .25208821473 .7136847825 H 7.2603389676 6.6027616404 4.9055090913 H 7.588429936 9.302098572 3.451903892 H 8.7231404818 .64297609074 .6459724307 H 7.08532909269 .07429381465 .126768196

${ }^{3} 13_{\text {DFT }}$

$\mathrm{E}^{0}=-3352.951848 ; \mathrm{G}^{0}=-3351.687552$

$\mathrm{E}^{\mathrm{0}}{ }_{\mathrm{Et} 2 \mathrm{O}}=-3353.77122 ; \mathrm{G}^{\mathrm{o}} \mathrm{Et2O}=-3352.506925 ;$ Charge $=0 ;$ Multiplicity $=3$

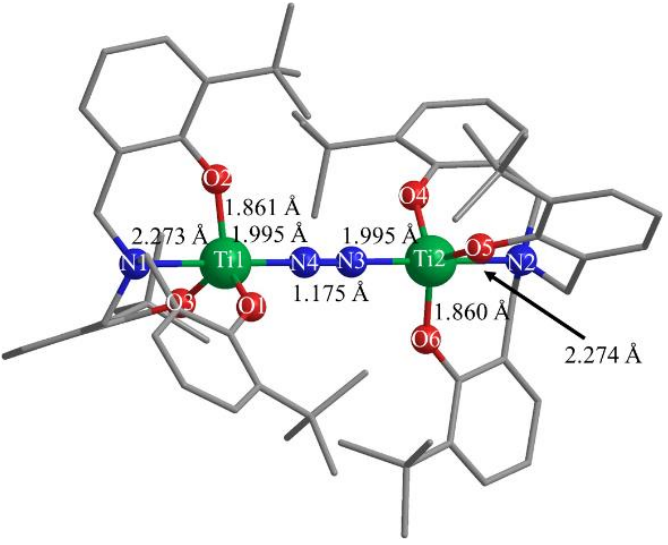

Ti $-0.78103812921 .0073607582-2.2154453819$ O $0.38526431110 .0906481124-3.3385350786$ O - $2.54411650560 .4227292879-2.0976556195$ O $-0.40536102372 .7833100332-1.8055509527$ N - $1.50659490071 .9079816538-4.17196426$ N $0.227240928-0.24929982630 .5140422549$ $\mathrm{N}-0.14371883880 .2170613923-0.498482503$ C $-3.67896502080 .3640714385-2.811942424$ C $0.2355983440 .3196408279-6.9581070015$ C $0.78359753050 .0348967046-4.6189573124$ C - $2.88947416012 .4358967689-3.975629366$ C $-3.91667352641 .3696121412-3.7668050149$ C $-0.13970171440 .390340079-5.6189766434$ C $-0.66509461854 .2096439165-3.6668035328$ C $-0.82782460355 .4919170428-4.1848926992$ C $-1.50727670110 .8458302099-5.2213607276$ C $2.1080661266-0.3377449013-4.939018533$ C $-0.5866064484 .0278861842-2.2739809872$ C $3.1495433333-0.5553900313-3.8401168977$ C -5.0962044904 1.3506313354 -4.5066319604 C $1.511927438-0.1056304841-7.3003469205$ C $2.4286059466-0.4135384992-6.297304846$ C $-6.03210591940 .3472334897-4.2962910827$ C $-0.9054882486 .5822849332-3.3292069832$ C $-4.5851998405-0.7035496008-2.6265835197$ C $-5.7630098009-0.6656579728-3.3782093836$ C $-0.87097461076 .3813760169-1.9508959973$ C $-0.58528056843 .0158666694-4.5637409663$ C $-0.73135472595 .1128132707-1.3809906951$ C $3.3303923940 .76564612-3.0712376675$

C $-4.2373340809-1.8778874399-1.7102052599$ C $4.5103213087-0.9389694356-4.4260369257$ C $-4.0005059066-1.4128800544-0.2651993672$ C $-2.9744740448-2.5683185084-2.2562866213$ C -0.82024791824 .88037485010 .1282902555$ C - $5.358385471-2.9193621585-1.6842758838$ C $2.7269031181-1.6738178001-2.8751923052$ C 0.44516863684 .19721608360 .6688665123 C -2.05583866844 .00869276820 .4164572279$ C -0.99711231476 .19696759260 .888199235$ Ti $0.8605007441-1.03978955642 .2324988753$ O $1.0837907485-2.78174055931 .6189284815$ O 2.36894446220 .03072100932 .4318866747 O - $0.6501426192-0.64216212033 .2429064152$ N $1.5877946146-1.93732849184 .1915469353$ C 3.46514908020 .16798335093 .1937270147 C $2.4820718106-5.43493701493 .6634139929$ C $1.3552486892-4.02130418472 .0558583645$ C $2.1287954717-0.84413958645 .0527372559$ C $3.3972854601-0.24304381614 .5373331659$ C $2.1638603242-4.16151410443 .1983741453$ C $-0.5997888534-1.65269296365 .3739102608$ C - $1.0674619976-1.71922771466 .683850647$ C $2.658588734-2.93542275293 .8970562805$ C $0.8081436442-5.14551578321 .398507913$ C $-1.103811647-0.66721503864 .5056935336$ C $-0.2022733437-4.97547445630 .262756183$ 
C $4.5111070841-0.09202327365 .3593606541$ C $2.0080708306-6.55654929982 .9967511914$ C $1.1744285975-6.40113720331 .8911744768$ C 5.67691495420 .46703233264 .8538380333 C $-2.0298793428-0.82093057327 .124158084$ C 4.65948352110 .679254042 .6392587415 C 5.74203806860 .83042048363 .5103066284 C -2.48835433650 .17560824056 .265040465$ C $0.4384793275-2.60471427234 .872311933$ C -2.03487584550 .29821864234 .9486854979$ C - $1.4425770414-4.25064865230 .8155899389$ C 4.76942299620 .96308799971 .1402146765 C $-0.6636148527-6.3285777551-0.2835144174$ C 3.73549546532 .00515096120 .6866414508 C $4.5592652271-0.35630433620 .3756314884$ C - 2.44508813351 .46425528784 .047483103 C 6.15625927641 .49447819170 .7711057335 C $0.3951853576-4.1802664637-0.908316631$ C -3.0930625709 0.97008085752 .7449252474 C -1.19228777452 .30001864363 .7294032557$ C -3.44961917882 .38434540354 .7449379899$ H $-0.48194797730 .6011640748-7.7265288396$ H $-3.16433932783 .0513711152-4.8477304851$ H -2.8441758589 $3.1143793134-3.1128881992$ H $-0.89350388425 .624918041-5.2631364795$ H - $2.04706579841 .2189399467-6.1070219567$ H - $2.09898473460 .0117973491-4.8200032098$ H -5.2713377384 2.1284756977 -5.2475172168 H $1.8060815483-0.1754077674-8.3431167156$ H $3.4313736879-0.7090633954-6.5881117572$ H -6.9594121076 $0.334361845-4.8608590948$ H - $1.01889648917 .5857901739-3.72783123$ H -6.49297731 -1.4593213709 -3.2568741857 H $-0.97106779377 .2445816076-1.3009675098$ H $0.42813207742 .591960821-4.5674384161$ H $-0.8068794313 .3148540772-5.6012492835$ H $3.5268508111 .5953168543-3.7596259435$ H $4.18502147890 .6955845026-2.3902768948$ H $2.45094517551 .0175318351-2.4711603827$ H $4.463068142-1.8719176156-4.9977574532$ H $5.226172043-1.0885312784-3.6113294172$ H $4.9148916647-0.1589553192-5.0797356896$ H -3.1432626074 - $0.7423221396-0.1850049676$ H -3.8146779448 -2.27782857910 .3819801447$ H $-4.8791718501-0.88927964230 .1270239638$ H -3.08440263 - $2.8003559076-3.3216363127$ H -2.8014931745 -3.5136234943-1.7315554154 H -2.0789913337 - $1.9526866042-2.1286756071$ H $-6.2986794233-2.5016230505-1.3090647263$ H -5.0744735425 - $3.7402875221-1.0179070551$ H -5.5472909668 -3.3495240497-2.6735890853 H $1.8159607046-1.423315226-2.3289543636$ H $3.5208124044-1.8564035273-2.1418379015$ H $2.5528878286-2.6114533105-3.4142244217$ H 0.58168330673 .19743239940 .2532769548 H 0.38501731624 .10422853481 .75938002 H 1.33854274334 .78687088650 .4361239785 H -2.950072657 4.428160639-0.0581677468 H - 2.24717243 .96459554951 .4936786295 H - 1.92841489542 .98127810230 .0624167893 H - 0.15438242426 .8785906980 .7311501764 H - 1.05655851535 .99021979521 .9616800981 $\mathrm{H}-1.91615520216 .71981538510 .6028470084$ H $3.103507395-5.53643578694 .5511904571$ H $2.289578985-1.2369676246 .0700107044$ H $1.3365519041-0.0869422965 .1288455904$ H - $0.6693581185-2.48034565957 .3523063344$ H $3.1522045631-3.21888855274 .8409777395$ H $3.4121428939-2.41399345393 .2911991252$ H $4.4540765536-0.41717282586 .3964910788$ H $2.2634208926-7.55198537263 .3470222025$ H $0.7874208029-7.29213039071 .4075817187$ H 6.5443033260 .59899227355 .493386907 H - $2.4078085049-0.87590273138 .1404922074$ H 6.67526709591 .23203599423 .1290629445
H -3.2141383945 0.88840160476 .6427221294 H $0.010383555-3.30685140844 .143962639$ H $0.8220477783-3.21141414945 .7088391532$ H - $1.2370526135-3.20307422651 .0551616972$ H - $1.8137535477-4.74453557211 .7206576537$ H - $2.2519902933-4.26680212280 .0783612494$ H $0.1685040088-6.9120013955-0.6917634964$ H - $1.3804910382-6.1662078808-1.0948744214$ H - $1.163020375-6.93505197180 .4795257526$ H 2.711281371 .65307323150 .8214510266 H $3.8766795372 .2359802502-0.3755562413$ H 3.84986157312 .93998569021 .2461056157 H $5.2111253699-1.14480875710 .76831421$ H $4.8052106501-0.2252025951-0.6832543001$ H $3.5241690648-0.70608123530 .4313525851$ H $6.19492147931 .6881069117-0.3057479966$ H 6.3853675372 .43529131181 .2828168063 H 6.95030443190 .77725497841 .0046638662 H $0.653983722-3.1592407604-0.622504395$ H - $0.3251819536-4.1281402252-1.7328522399$ H $1.2998310352-4.6658733357-1.2901616257$ H - 2.39695071410 .39507235172 .1318376484 H -3.4398848703 1.82366025582 .1510928964 H -3.962987887 0.3382725438 2.9547927109 H - 0.655851992 .56676940294 .6469736172 H - 1.47430968513 .23362388653 .2316058674 H - 0.50014271761 .7705774043 .0677841148 H -4.3724946665 1.85817919225 .0112322844 H -3.72074274953 .20243335384 .0698066392$ H -3.0381048881 2.8327688086 5.6554308974

${ }^{1} 13 \mathrm{DFT}$

$\mathrm{E}^{0}=-3352.938228 ; \mathrm{G}^{0}=-3351.669678$

$\mathrm{E}^{0} \mathrm{Et2O}^{-}=-3353.756699 ; \mathrm{G}^{0} \mathrm{Et2O}^{-}=-3352.488149$ Charge $=0 ;$ Multiplicity $=1$

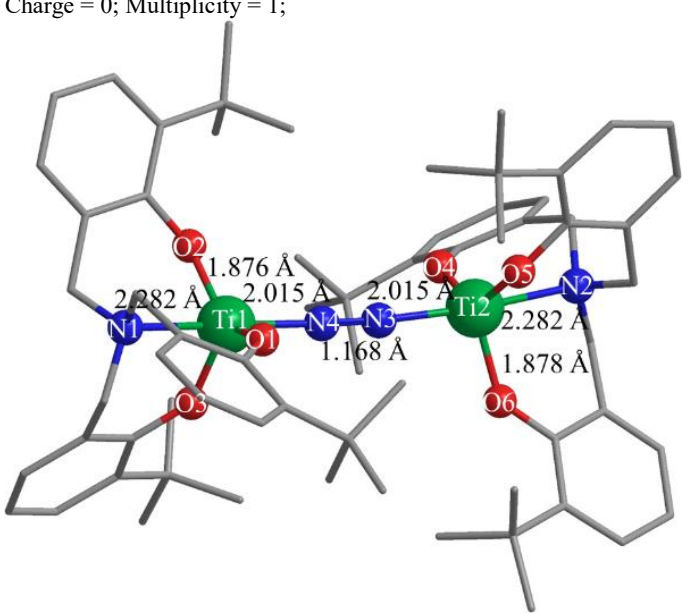

Ti - $0.70401493131 .2479994609-2.1446220905$ O $0.38968094440 .353569389-3.3813766244$ O $-2.41257336480 .5723178443-1.9423016218$ O $-0.00132241722 .9584883604-1.8311171993$ N - $1.66029760912 .2051941348-3.9819507718$ N $0.1274506302-0.21686973690 .5533650095$ $\mathrm{N}-0.12060251460 .3144269351-0.4569421631$ C $-3.55574393050 .3815903847-2.6254021582$ C $0.00392268720 .9076321081-6.9575658127$ C $0.68482457310 .3964637522-4.6878665883$ C $-3.06669719342 .5962267815-3.6522347349$ C $-3.97060747141 .4193545152-3.4776245125$ C $-0.28961799640 .8542429324-5.5966871243$ C $-0.91187942374 .5006951927-3.3609873516$ C $-1.38521863685 .7751247705-3.6614762786$ C - $1.6515754971 .2137683362-5.0977574807$ C $1.9597268714-0.0222910796-5.137595445$ C $-0.40590668434 .2220513903-2.076604782$ C $3.0320462131-0.4766399471-4.1460003575$ C $-5.15666833651 .2880369056-4.1936609532$ 
C $1.24459009110 .4932741398-7.42005168$ C $2.19847789270 .0367996625-6.5129066629$ C $-5.91848796710 .1341958148-4.0632436748$ C $-1.34185993566 .7751679896-2.7007303265$ C $-4.2673951353-0.8325117049-2.5365455279$ C $-5.4614247554-0.9087811594-3.2607805168$ C $-0.80834047996 .4965328225-1.4451529878$ C $-0.89700553353 .4168894372-4.3875701457$ C $-0.32042804275 .2340685789-1.0923133251$ C $3.40328415870 .7049126371-3.2362858204$ C $-3.718095203-2.0095748576-1.7319783245$ C $4.3076228298-0.9251929173-4.8630476464$ C $-3.6082965226-1.6312788087-0.2501820689$ C - $-2.3400793229-2.4199806824-2.2828279155$ C 0.33585127684 .97792771490 .264710705 C $-4.6329798319-3.2322938083-1.8312126714$ C $2.5407520681-1.6594201529-3.2944123916$ C 1.81009478614 .62082040980 .0347777575 C -0.36434675583 .84221364821 .0231337689$ C 0.29159544546 .21751139621 .1611264443 Ti $0.6194248778-0.99092827752 .3472612383$ O $0.9367030082-2.76974159081 .8384907894$ O 2.10399495940 .10756891012 .4266930507 O - $1.0423817842-0.88984460993 .212518728$ N $1.407156915-1.64381937194 .3865696295$ C 3.26651921470 .25606604193 .087555598 C $2.3027494545-5.17666037224 .2020202463$ C $1.2441084344-3.94638791732 .4016285412$ C $1.9741856513-0.45293205665 .0937183169$ C 3.24315660970 .03714719384 .4756841749 C $1.980529081-3.96098023673 .6026944157$ C $-0.7709179574-1.23623276245 .5264259112$ C $-1.120007722-0.95395357296 .8443528603$ C $2.4681681636-2.67385622344 .1847203496$ C $0.8345643957-5.15224976071 .7844129007$ C $-1.4528176163-0.60753669544 .4665773585$ C $-0.0045850313-5.13394452630 .5057841446$ C 4.41042264960 .19565786015 .2164601392 C $1.9181259854-6.37039808543 .6087381922$ C $1.1962891049-6.34407679192 .4173446105$ C 5.58795121540 .5658072674 .5798721256

C - $2.151425762-0.06590166497 .1132007549$ C 4.46432380980 .55334034042 .4052229458 C 5.60611183650 .72001418713 .1955126044 C -2.83922112090 .52548062926 .0567446778$ C $0.3039481876-2.220299445 .2029811518$ C - 2.52820485240 .27673279694 .7159285256 C - $1.3604623987-4.4783723770 .8111878297$ C 4.50318316610 .63041084260 .8796830223 C $-0.2812658982-6.5494836085-0.0061040147$ C 3.60018220761 .76669879990 .3855392452 C $4.050936895-0.71254031250 .2774237824$ C -3.35348384070 .87577729593 .5765975989$ C 5.91857663020 .90747374650 .3679876493 C $0.7077913926-4.3662050657-0.6207524768$ C $-4.0472843739-0.26753187452 .824901376$ C - 2.47660257171 .68084721942 .6079087396 C -4.44054078541 .81947481934 .0959157038$ H $-0.75503363411 .2692935447-7.6491638137$ H -3.4515435793 $3.2475206029-4.4539313297$ H -3.012720772 $3.2116056075-2.74365532$ H - $1.78250687115 .9722761157-4.6555589861$ H -2.2611836829 1.6083631845 -5.9273618087 H - $2.16672133940 .3187105544-4.7264702425$ H -5.468126817 2.0899137152 -4.8602004054 H $1.47591445990 .5271005903-8.48028413$ H $3.1633555906-0.2787198672-6.8961345856$ H $-6.85099403770 .0280201571-4.6090084418$ H - $1.71298163877 .7707019072-2.9240983328$ H -6.0506434129 -1.8186666938 -3.2124784793 H $-0.77034201577 .2970554033-0.7139281427$ H $0.12925653093 .0837455839-4.5881299833$ H - $1.30792849353 .7919421313-5.3396439742$ H $3.70468266861 .5767328357-3.8274242034$ H $4.24878493650 .4352624989-2.5920725577$
H $2.56991485470 .9988389129-2.5919478079$ H $4.1242841701-1.7671402031-5.5390158282$ H $5.0438274076-1.2522367603-4.1218718869$ H $4.7637711123-0.1158871622-5.4430154533$ H - $2.9385336046-0.782703166-0.0867019771$ H - $3.2304703912-2.47932680070 .3310724529$ H $-4.5915205313-1.36730156520 .1526836148$ H - $2.3790742761-2.5751899876-3.3670920365$ H - $2.022080055-3.3627637685-1.8262478257$ H - $1.5622377508-1.6810963-2.0725961857$ H -5.6351039673 -3.0301331036 -1.4387072394 H $-4.2090798338-4.0498941909-1.2390202622$ H $-4.7349408182-3.5896325523-2.8615562526$ H $1.6918871495-1.3906148461-2.6622118684$ H $3.349757064-2.0108429035-2.6430308729$ H $2.241030694-2.5005241841-3.9291722024$ H $1.91616509963 .7288898067-0.586473354$ H 2.30412178244 .42650125860 .9933687243 H $2.33827156275 .4457881971-0.4553622496$ $\mathrm{H}-1.42112834664 .07689084591 .191155223$ H 0.10460664183 .70595695882 .005396558 H - 0.30674984082 .88709418530 .4977303496 H 0.82498412657 .06632218160 .7208395719 H 0.7740825635 .98922908282 .116586344 H - 0.73449786246 .53390457581 .3776292323 H $2.8662100592-5.17480984915 .1334882822$ H $2.1497665991-0.7167512646 .1494354924$ H 1.18921182350 .31572904385 .0858090455 H - $0.5765185566-1.44037935867 .6524080835$ H $2.9648143092-2.86510593475 .1503245477$ H $3.2226769744-2.22359184463 .5270986402$ H 4.38945189030 .01418237626 .2892622792 H 2.172445982 -7.3203918936 4.0687232999 H $0.9027398195-7.28919042341 .9722661616$ H 6.50077514020 .70312388665 .1513732934 H - 2.42799468720 .16432686578 .1375070593 H 6.54852920630 .9632590982 .7159002562 H -3.6510775612 1.20627766556 .2898519964 H - $0.0984029753-3.06433947814 .6284680991$ H $0.7300353601-2.63414858926 .1320070048$ H - $1.2519390543-3.42021197731 .0655862915$ H - $1.8612769892-4.98245131271 .645119432$ H - $2.0208804068-4.5496113927-0.0613493399$ H $0.6424632328-7.0895065487-0.239649736$ H - $0.8715586908-6.4929939369-0.9262558045$ H $-0.8523011213-7.14638491320 .7129205278$ H 2.55638969681 .62452522050 .6783377928 H $3.6403840441 .8360723609-0.7068833877$ H 3.93570857122 .72744779120 .7894194688 H $4.6093462935-1.54850664080 .713973015$ H $4.237730109-0.7189590899-0.8012776998$ H $2.9841761355-0.90373319160 .4213205798$ H $5.90379732610 .9591834875-0.7256174643$ H 6.31020703241 .86206425860 .7348511025 H 6.6223853210 .11754347930 .6514583142 H $0.8408245104-3.3088489779-0.3821902436$ H $0.1224602346-4.4334746915-1.5454682315$ H $1.6943478573-4.7969088663-0.8243507034$ H -3.323976378 - 0.97618502192 .4161864519 H -4.6378247513 0.13194901331 .9927659052 H - $4.7260966993-0.81385121483 .4886026573$ H - 1.96328946582 .49607804213 .1294739951 H -3.10126988892 .12987375681 .825917653$ $\mathrm{H}-1.7234550811 .06588466542 .1119476778$ H -5.1546524519 1.30870738944 .7501158634 H -5.0044552697 2.2212821208 3.248380094 H - -4.02113844772 .66918316074 .6454378305$

${ }^{3} 14 \mathrm{DFT}$

$\mathrm{E}^{0}=-3352.929734 ; \mathrm{G}^{0}=-3351.665025$

$\mathrm{E}^{0}{ }_{\mathrm{Et} 2 \mathrm{O}}=-3353.752001 ; \mathrm{G}^{\mathrm{o}} \mathrm{Et2O}=-3352.487293 ;$ Charge $=0 ;$ Multiplicity $=3$ 


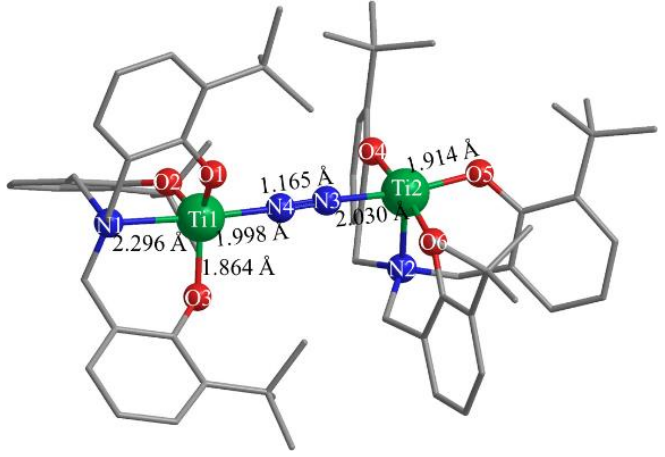

Ti 16.08605466335 .343683179610 .3545012167 Ti 15.42339432247 .730038195 .7954797696 O 15.42709929383 .67289122189 .8568080185 O 14.80345953046 .426219040511 .1902465226 O 17.87761653885 .795496925410 .5247299921 O 15.27903813028 .80654535564 .2197880753 O 16.43505320466 .38098193384 .9524919703 O 14.26255950848 .89826344936 .6997719509 N 16.38897522624 .411417914712 .4306334725 N 15.90620014396 .24281456628 .5794935275 N 15.7891035716 .81002811227 .5682282866 N 13.69671981626 .54819233485 .2282760674 C 15.5475994493 .185463555612 .5571297587 C 15.99315146875 .405253865813 .4742529554 C 15.77808208592 .415101401410 .1916338691 C 15.88235524892 .117761727711 .5636183042 C 16.2577656570 .836787466311 .9607946309 C 13.9999440066 .300669184312 .2571601672 C 14.54556498345 .779450024113 .4458147248 C 16.02146829821 .43982649979 .1995526196 C 17.82262827754 .037233149812 .6232898391 C 19.66357883965 .428847993913 .6528580768 C 16.38956471240 .16899368179 .65247616 C 18.76738642115 .196975469512 .6129133229 C 18.75920952776 .059437421211 .5031437515 C 20.54701025616 .498104276813 .5788040697 C 13.73920783265 .648952837514 .5742722053 C $16.5125607098-0.138491518511 .0058825748$ C 15.24469901548 .22976272143 .0063712877 C 15.87107163641 .76480871637 .7109024315 C 12.40902986836 .042646776714 .5257262225 C 12.64191200846 .686702021312 .1751774605 C 12.0701972168 .16633288576 .360681771 C 16.06954956498 .67704446591 .9479229636 C 14.01675652295 .08986650025 .3162844455 C 16.33828711235 .18612045824 .3665633719 C 11.88037405696 .547800651813 .3390628684 C 12.03604932567 .183897873110 .8587541908 C 14.331757257 .16608171922 .8204893104 C 19.63047459497 .165644871411 .4093866239 C 20.52002146897 .346371094912 .4731764657 C 12.59204703386 .77398886266 .2239575991 C 15.08248192984 .54868012774 .4217730393 C 12.96908207129 .21246709886 .6570646657 C 16.86895601932 .85318868677 .2813278787 C 14.43520018962 .22970134127 .4220292759 C 14.88884044193 .33652053253 .7651455803 C 16.04862055577 .91694786890 .7744198856 C 16.13855693380 .53883458036 .834184936 C 14.34752195246 .44722203511 .6281641919 C 13.25776636736 .93734998163 .8470952717 C 15.23234060226 .79812388930 .6173480778 C 12.78687190098 .409633698410 .3144146743 C 10.70019590198 .4207232766 .3258042397 C 16.84777201739 .99460093052 .0501693655 C 12.508735398910 .52520444396 .9157729251 C 17.43714008674 .56987288013 .7228930888 C 11.126469719710 .72356364426 .8747100691 C 12.07718022096 .05447569389 .8159356199 C 17.19390451093 .34861800773 .0878037296
C 13.490918747711 .65569163747 .2244100155 C 15.94178578782 .73827169293 .0846205226 C 17.600770937510 .30299900880 .7545194494 C 15.836955746511 .12803853932 .2908998386 C 19.7770716847 .37358947188 .891978121 C 19.5653160868 .124166207810 .2168684314 C 17.883933268210 .00364224073 .1832023064 C 10.56977482867 .589129427311 .0318834447 C 14.429263743711 .87149420066 .0265669628 C 10.22550992119 .69838405116 .5885837279 C 18.82811139925 .19916032413 .7652088855 C 12.766067618112 .97772737767 .4847970418 C 14.306974073311 .32257084028 .4833271237 C 18.20095818638 .834403083610 .197180792 C 20.64437818519 .205272165810 .3097158765 C 18.80135165156 .56530967523 .0708090559 C 19.27502338225 .35795160545 .2283979333 C 19.87035347624 .33300629383 .0564819434 H 14.50720765843 .513123701712 .4367895994 H 15.64741155522 .792796153513 .582834783 H 16.62806839136 .286373390813 .311257782 H 16.257010684 .999381025114 .4651380791 H 16.34379793810 .612889987113 .0225745802 H 18.06434300843 .32980715211 .818935837 H 17.91611050293 .485623849113 .5738082308 H 19.66444285624 .764228228914 .5148252652 H $16.5902364081-0.61221440978 .9265170993$ H 21.25302704696 .681798755314 .3829021613 H 14.1660441465 .240982928815 .4888803355 H 16.8062283748 - 1.138972867211 .3085029535 H 11.77669770695 .950005205215 .4034555322 H 13.0812040444 .53363890035 .1442180829 H 14.2935397444 4.9149889238 6.3667607256 H 10.83434145936 .836482890713 .3242225912 H 21.21443951998 .179969162112 .4464862647 H 11.7641074336 .09422246735 .9686803228 H 13.00705962386 .42881879147 .1815055005 H 16.68584448453 .81211590717 .7747669568 H 16.79800627663 .01933250136 .1989141691 H 17.89790980092 .54932548417 .5034995977 H 13.71175910411 .4535234357 .6939606559 H 14.32466779332 .42993270186 .3492662042 H 14.18132464383 .13809421257 .9750357819 H 13.91093028612 .85910125193 .8078089593 H $16.69006868748 .2050027056-0.0525932472$ H 17.16157987970 .16530828046 .9494695214 H 16.00653770930 .81388868245 .7823236319 H $15.4487426972-0.28322012867 .0533654467$ H 13.66101528715 .61206540391 .500929234 H 12.68993543837 .86648216443 .9830885146 H 12.55594254926 .16824936463 .4906969017 H $15.26412015926 .2290165565-0.3066132466$ H 12.78731343019 .224216817711 .0470338149 H 12.28772100098 .77889120769 .4091826357 H 13.82440062678 .182395232210 .059811562 H 10.0125414787 .60598473226 .1055957696 H 10.731046578411 .7149340467 .0713448409 H 13.10258506645 .78709137019 .5402549303 H 11.55070386896 .37492577288 .9084041672 H 11.57984379935 .152203338410 .1883109973 H 18.01106453752 .85093393012 .5758704442 H 15.79789094581 .79384140212 .5687779616 H 18.35165441839 .53801617240 .5260298921 H 18.125308012511 .25840649970 .8564554758 H $16.927247678510 .3873603443-0.1045367434$ H 15.111428775711 .18308114931 .4724364163 H 16.355675539212 .09225960942 .3464316368 H 15.289572234610 .98158259263 .2244276035 H 20.75146949066 .87360134228 .8748157148 H 19.75658254748 .08512577858 .0574019051 H 19.00691686356 .62117040468 .7135525573 H 17.43338040259 .78534632094 .1535488325 H 18.344993059410 .99618684573 .2458890393 H 18.6837104179 .28110442792 .9996259401 H 9.94035664556 .748774720411 .3437993097 
H 10.18170888037 .955754388510 .075631778 H 10.45156970998 .393487250511 .7656113553 H 15.000450161310 .97485192815 .7779757028 H 15.136486112712 .68031558886 .2443108816 H 13.860124937812 .15810458415 .1355711868 H 9.15970345349 .90345932956 .5684365758 H 12.184708101213 .3068616296 .6169834363 H 13.501436550813 .75870321297 .7021584016 H 12.090446291812 .91420832118 .3444697383 H 13.650716513611 .21599611989 .3537321833 H 15.013315677512 .13351188528 .6951454728 H 14.87378731210 .39572010238 .3760929696 H 17.36757067138 .134384563410 .0985334838 H 18.15210168219 .53008524119 .3507035353 H 18.04826284389 .415198530411 .1129628142 H 20.52810611399 .829716378411 .2017148667 H 20.57612240389 .86416754119 .4385131015 H 21.65266025618 .777889991810 .3218919155 H 18.52426481336 .46584147442 .0152780845 H 19.79161413147 .03454662773 .1196440646 H 18.07949160247 .23617352683 .5373711386 H 18.57766028585 .96628237015 .8089344009 H 20.26059691835 .83597307915 .2730712912 H 19.35577219834 .3808269115 .7172433275 H 19.96047775253 .34077039263 .5114154561 H 20.85024718534 .8160160663 .124693782 H 19.6425721874 .20223071071 .9933156447

${ }^{1} 14_{\text {DFT }}$

$\mathrm{E}^{0}=-3352.933335 ; \mathrm{G}^{0}=-3351.663155 ;$

$\mathrm{E}^{0}{ }_{\mathrm{Et} 2 \mathrm{O}}=-3353.753913 ; \mathrm{G}^{0}{ }_{\mathrm{Et} 2 \mathrm{O}}=-3352.483733$; Charge $=0 ;$ Multiplicity $=1$;

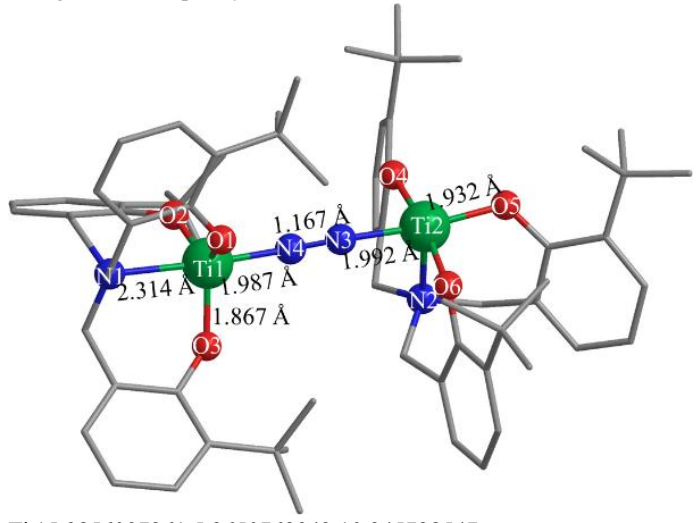

Ti 15.98560972615 .365076294310 .345728547 Ti 15.44256835757 .78461435275 .8418176699 O 15.46207771793 .66237643539 .7868772426 O 14.80453756656 .436485612911 .2543749222 O 17.84077798455 .561696247510 .3883898436 O 15.32066601568 .83664628634 .2264551921 O 16.50525984646 .42575276915 .0882682289 O 14.26372280648 .94654633546 .728379586 N 16.2907317414 .383507458312 .4186481733 N 15.70856002476 .30936124948 .619445468 N 15.66785565656 .87391830957 .5988610959 N 13.73514275226 .57122136745 .2649461617 C 15.41168199543 .179697789212 .5082760069 C 15.92808460355 .35210911513 .4956490019 C 15.76277060032 .40540547310 .1580858277 C 15.76923918292 .103861303411 .5324895961 C 16.09194344090 .8148115611 .9509501899 C 13.9653720796 .342252957312 .3065165787 C 14.4941435765 .780684581213 .4815998965 C 16.05018563151 .42485903419 .182134549 C 17.71408375673 .965692070912 .5988487656 C 19.5650587355 .381993097113 .5731286012 C 16.36535867490 .14762048579 .654706036 C 18.68302125975 .100520903512 .5334956424 C 18.70248921265 .890534980111 .3696456274 C 20.46278481076 .434182826213 .4510587098
C 13.68013336975 .659015826814 .6044114454 C $16.392445322-0.163506919411 .0131492681$ C 15.27648346268 .23993255063 .026857287 C 15.97277170711 .74950368177 .6877783692 C 12.3652675956 .103431005114 .556653651 C 12.62723130276 .776969597112 .2167216783 C 12.08256377078 .15740564636 .4097478095 C 16.09286409528 .67179425191 .9527076235 C 14.07427564415 .11762206895 .3508039476 C 16.42585659065 .24136468164 .4728383485 C 11.85833215376 .646671367113 .3784351846 C 12.04273342527 .31023716310 .9058982923 C 14.36711272127 .16922972742 .8572529194 C 19.58122599486 .98816357311 .2338492773 C 20.4566778786 7.219407702612.2998503847 C 12.62371250486 .7729932086 .254650534 C 15.17208687454 .59819414614 .4819533775 C 12.96080893959 .22069563196 .7080614004 C 16.9456679412 .87646739747 .2978894192 C 14.53525580562 .16206212577 .3343493146 C 15.00178543433 .40042399513 .7936877274 C 16.07881819037 .88631485190 .7964514666 C 16.32663042360 .53312952496 .8283349314 C 14.38734210976 .42583276481 .6796753197 C 13.2924074356 .95618358893 .8848845719 C 15.2717546966 .75729493660 .6626108901 C 12.84625288348 .506254884210 .3728140136 C 10.70764369088 .3862588436 .3858810236 C 16.851364182310 .00314307472 .0208642367 C 12.475360639410 .52333707476 .9757216584 C 17.5401299994 .65159314543 .8305262085 C 11.089272917410 .69449744896 .9467997165 C 12.0335763776 .18636008369 .8564630315 C 17.31946340243 .44275140353 .16356658 C 13.437262177911 .67590642657 .2678031658 C 16.07336201952 .82235000543 .125192007 C 17.581938192710 .30153022880 .7100297442 C 15.826427335111 .12533825432 .2543839758 C 19.71332390617 .11343486648 .7054675673 C 19.52641872327 .904819440510 .0085797731 C 17.901574387710 .0486017773 .1395488194 C 10.59597592667 .777430721211 .0857916199 C 14.348569491211 .91183007156 .0526818196 C 10.2083499779 .65191780396 .6614549835 C 18.92306331345 .29616146633 .8958581671 C 12.68642372412 .9810946647 .539923387 C 14.284677142411 .36635535228 .5119093358 C 18.1717747338 .63300166949 .9833890779 C 20.62114121548 .973276820310 .0568338813 C 18.88390823186 .6774882973 .2327543561 C 19.35727587775 .43198517765 .3636860424 C 19.98035801164 .45867809333 .1751307145 H 14.38537050223 .53404824312 .342570409 H 15.4542773632 .784943383813 .5371878771 H 16.59378125616 .215815559813 .3664394855 H 16.17323827334 .901542230614 .4719371729 H 16.10521847440 .588695543713 .0156902622 H 17.92081628523 .232911710211 .8096365444 H 17.80621399093 .439037569813 .5634089772 H 19.54279831094 .768879217914 .4722760513 H $16.5998449585-0.63641776078 .9420375531$ H 21.16016378736 .656504099814 .2529437896 H 14.08737506195 .218383006315 .5123185906 H $16.6475638726-1.169348973311 .3325040094$ H 11.7266427096 .019170605215 .4305825308 H 13.15268661184 .54795889885 .1467355709 H 14.32695747724 .93965943846 .4062424144 H 10.82367313186 .97289082613 .365169192 H 21.15655607538 .046377061712 .238027616 H 11.80220324896 .08997549915 .9849247654 H 13.0352477352 6.418512045 7.2098748878 H 16.65291425083 .84605239747 .7094652525 H 16.974251322 .97984409926 .2059290384 H 17.96370989082 .65774703667 .6391562707 H 13.8306166471 .35490504347 .5620402113 
H 14.46656629072 .37602119766 .2610898606 H 14.22370893263 .05243052947 .8873295824 H 14.02493077582 .91912577113 .7997212472 H $16.71610773428 .1635345814-0.0376634889$ H 17.35155655190 .18923540357 .0046025529 H 16.24742148350 .80543627215 .7705894962 H $15.6494241869-0.30907739087 .0057875076$ H 13.70302855895 .58608949821 .5695591171 H 12.73328733747 .89120270674 .0186133199 H 12.58180273276 .19235210073 .5328195092 H $15.30751949816 .1690894138-0.2492132485$ H 12.87354243939 .317761080211 .1082263877 H 12.36934086488 .8968134199 .4650740499 H 13.87657957258 .243008102410 .1229228345 H 10.03493034457 .56005275056 .1622871034 H 10.675123898611 .6768907537 .149673188 H 13.0443901755 .86223406789 .5856003465 H 11.53670981236 .54159985638 .9454025401 H 11.4820886225 .311891299910 .2184876719 H 18.14915318632 .9649803912 .6530907798 H 15.94675200411 .88825047562 .586352231 H 18.34457858959 .54711738530 .4854893651 H 18.089500526311 .26846702210 .787735034 H $16.896021262110 .356881849-0.1416390242$ H 15.088022899111 .15254789231 .4460161221 H 16.330666176112 .09847205332 .2826616599 H 15.295689200210 .98811171223 .1987185233 H 20.6823567586 .60270836218 .6897092409 H 19.68796448077 .80020216887 .8506890809 H 18.93406104146 .36395119918 .5589117365 H 17.46781803059 .82792747034 .1170422403 H 18.3409376207 11.05200397793 .1861514308 H 18.71491716819 .34249557662 .9514077868 H 9.931159766 .962058160911 .3899927887 H 10.22252117898 .169269426110 .1341485834 H 10.51410747938 .57905630711 .8273652437 H 14.940139397911 .03034282355 .7980634863 H 15.036479363512 .74084896016 .2555823788 H 13.756569751312 .17903659695 .1706804751 H 9.13825528429 .83458041926 .6495505182 H 12.085250341513 .297616966 .681035012 H 13.407110047713 .77833413857 .7470868257 H 12.02493654312 .90190828898 .4093615673 H 13.648233363211 .25106636729 .3959398906 H 14.976355957712 .19423111758 .7055768716 H 14.870880225110 .45239990388 .397506256 H 17.329139371 7.9399621642 9.908939374 H 18.11954185099 .30444344199 .1178122034 H 18.03656924559 .240189799410 .8848453511 H 20.51620251389 .635903739610 .9222265689 H 20.55999784619.59683921619.159530715 H 21.62317811128 .532128562710 .084312752 H 18.59723961526 .60315248662 .177560769 H 19.87269776477 .14946412673 .2844679643 H 18.16496592267 .33498911873 .7223147561 H 18.65185285276 .03036264475 .9439802365 H 20.34014159745 .91392193075 .4244046969 H 19.43765551154 .44863613175 .8404361003 H 20.08015470283 .45792739083 .609001841 H 20.95346138024 .95244241763 .2609416136 H 19.76154041234 .34855154642 .1077513965

\section{DFT}

$E^{0}=-4293.577244 ; G^{0}=-4291.858046 ;$

$\mathrm{E}^{0}{ }_{\mathrm{Et} 2 \mathrm{O}}=-6009.417898 ; \mathrm{G}^{\mathrm{o}}{ }_{\mathrm{Et} 2 \mathrm{O}}=-6007.6987 ;$ Charge $=0 ;$ Multiplicity $=1$

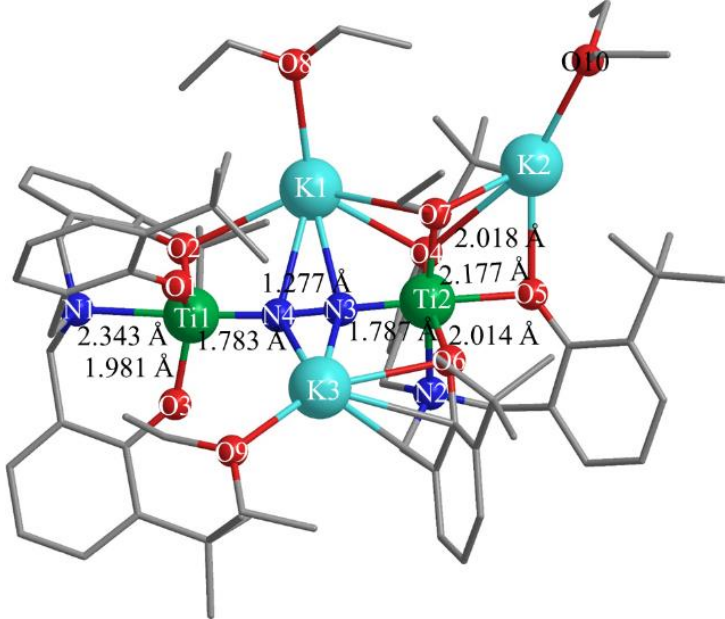

Ti 14.64112458245 .491157189410 .2588988163 Ti 13.71766446916 .69914182425 .6716298029 K 14.95266657298 .77105279088 .4071363822 K 12.13443301084 .58773615687 .7858743648 K 10.90186948296 .10644130393 .6668520801 O 13.52982800096 .889070598611 .1164189929 O 16.57225891875 .508803656610 .3296783204 O 13.83735997293 .712039744810 .0273742553 O 15.953206411410 .032549279610 .525611283 O 13.41507701796 .87971908573 .5234122722 O 14.31744726178 .62190859995 .6706814881 O 13.36210141764 .72794870975 .1687631575 N 14.8524857284 .807205009312 .4902644227 O 11.72919509886 .97939621345 .8729937575 N 14.37512963766 .04764391718 .5858458622 N 14.06724924086 .41166878217 .4006612276 N 15.8720320556 .22564366975 .1560988498 O 9.50852159994 .44985636868 .4825190815 C 14.99572073265 .990464262813 .3679357886 C 16.06411424943 .960950964212 .6394613038 C 13.11090294217 .286753710812 .3145982156 C 13.78597423996 .860405770713 .4804432463 C 13.36683246937 .295269766514 .7362828262 C 17.55840578795 .404115123311 .216752618 C 17.35356993764 .693859004212 .4210022609 C 11.98851628848 .154094103412 .4337102841 C 13.60944240264 .056787625212 .8218638528 C 13.36712542161 .563624193312 .9325308744 C 11.61888482338 .576156457813 .7130342163 C 13.55489436682 .712244556312 .1672847599 C 13.79844209752 .60744426210 .779128046 C 13.44463184050 .308569909612 .3447266806 C 18.37179267584 .628939043913 .3695486798 C 12.29346808888 .165578675814 .8603965606 C 14.23393082267 .63467266962 .8113786517 C 11.18351439588 .567575224611 .199530599 C 19.59707566725 .237326805613 .1317606058 C 18.82027622065 .997008324510 .941826468 C 15.50766656223 .6975338075 .115059658 C 13.75972173528 .60191507581 .873371705 C 16.87997642711 .109503192610 .4883731881 C 16.77297887437 .15108861665 .8834434539 C 15.44311512969 .31215563785 .594821666 C 19.80832656845 .900312697411 .9242992933 C 19.08545626156 .66588164039 .5890760105 C 15.63707040957 .45277318942 .9487175694 C 14.03692783751 .331865033810 .200470518 C 13.81508161270 .210039990611 .0050460449 C 16.17704015224 .88089496825 .7384316961 C 16.68780231698 .62819139375 .6289177851 C 14.11385607013 .68716311414 .8475352752 C 10.51141383127 .318387568210 .609338568 C 12.08436683579 .204506360410 .1300975512 C 17.88342458549 .34058380965 .5456189176 
C 15.304591014110 .040618613612 .898757264 C 15.95981002739 .303315700311 .7536026131 C 14.69206364749 .43677431541 .2542128912 C 10.08318598199 .574762946711 .5389813252 C 16.52664059298 .30469092832 .3008638043 C 16.13226488156 .24722362023 .690611046 C 16.06269788549 .32658618821 .4822440287 C 18.88840758895 .64128499828 .4600521575 C 16.27875373482 .56011697364 .8815042458 C 12.27573759558 .64140228531 .4987055103 C 13.55411085762 .52500555534 .2278181172 C 15.439297636210 .74093277665 .5574262747 C 14.36640772361 .40633061384 .0339479621 C 18.13437939357 .85218225559 .3741511952 C 16.668390715611 .40107693675 .4740542252 C 12.12766784192 .54996259453 .6812790434 C 17.886026211110 .72606533065 .4553746696 C 17.018483115111 .56398254129 .0575487653 C 11.96235081899 .69317654270 .4323848552 C 11.9017687917 .27280213850 .8912009818 C 13.82901738371 .9171245737 .7172481003 C 14.64770554171 .2015660448 .8015607516 C 11.37790483838 .96754787962 .7091768586 C 20.51429473457 .20136865459 .4828500512 C 12.12774150843 .55519287122 .5149854736 C 15.7151456281 .39537260234 .3782592568 C 14.124606247411 .52601391685 .5974778407 C 11.67550705531 .1991428913 .1218758878 C 11.09675592592 .9329384734 .7553143088 C 16.05432132931 .8273556228 .81982588 C $14.7910349659-0.26201846828 .3761740543$ C 10.42372491893 .500363359910 .4799154346 C 13.274965947411 .15396713654 .3800362255 C 11.25774401217 .74446103536 .9338725091 C 13.329910072411 .2025079526 .8748470666 C 9.20938277294 .11873173869 .8376372621 C 14.350439459813 .0387586395 .5808826616 O 8.34618399766 .79581364483 .0321110079 C 10.22415748948 .76034867486 .4823680969 C 8.44726074685 .16617856837 .867285524 C 8.13094288364.5455479642.3018719411 C 8.7157930375.26680777096.3868158509 C 7.42526576915 .86526676452 .4803840343 C 7.76535235098 .07031985643 .3073279797 C 7.4331055798 .86457178752 .0634729231 H 11.73816161388 .51497063923 .6377063461 H 12.63471598174 .48818700082 .7878866922 H 15.85109450256 .561083219412 .9747482412 H 15.29386231285 .656880667614 .3786394932 H 15.97381375173 .138625529711 .9185170999 H 16.06791068353 .501897291113 .6442983955 H 13.90169707946 .946149265215 .6186354375 H 12.77219374714 .703572181112 .5184179609 H 13.53199625943 .932646041113 .9160323513 H 13.17663942911 .665974901613 .99996465 H 10.77271844859 .246337641113 .8275187942 H $13.2781025354-0.589526039312 .9319544219$ H 18.19323136584 .088032136214 .2980216378 H 11.97190448598 .513544955515 .8373201927 Н 20.38986197925 .188618838513 .8722773456 H 17.849887406810 .764582981410 .8869305614 H 16.539395195111 .932933548911 .1350517034 H 17.81369327126 .83369740375 .6893051157 H 16.59855379756 .93060366396 .9493282762 H 20.78055440526 .349807793711 .7457468359 H $13.9553886106-0.779917636610 .5826371008$ H 17.26939287984 .72414517765 .6847370563 H 15.92381332924 .95695010236 .805696739 H 11.2610445593 6.5919595748 10.2748033698 H 9.88198490337 .58537823369 .7496939206 H 9.87370899436 .837252432611 .3601601331 H 12.71475338599 .995585397310 .5558792571 H 11.47779274829 .64995287669 .3317070065 H 12.72092961798 .4328361099 .6954890783 H 18.82225670618 .78573345015 .5549967799
H 14.265221628210 .287015565312 .6603336969 H 15.834328852510 .965045174913 .148923277 H 15.29054029799 .409692204313 .7923277852 H 16.99954801589 .025205883511 .9987202746 H 15.41687461018 .374483954511 .5362966307 H 14.344360497610 .20201757220 .5647283714 H 9.35118138569 .167813975712 .2439829339 H 9.54311560819 .846459629810 .6254892081 H 10.490922584510 .495763063811 .9694269524 H 17.59668073358 .15789311432 .4478296472 H 15.62908897885 .35512347433 .2959333523 H 17.21585907836 .11792960053 .521424668 H 16.755782677510 .00534686480 .9945010992 H 19.54594474554 .7770315488 .602849792 H 19.13957995366 .09534020427 .4929377885 H 17.85649585935 .28398233898 .417154587 H 17.34199848552 .59800398945 .1160815673 H 13.94008481050 .51108962493 .5913955131 H 17.0970814835 7.5251492679 9.4800015867 H 18.27723859838 .28302837468 .3723035779 H 18.32185794468 .645110030610 .1077875187 H 16.682764486212 .48574617155 .4271132017 H 18.818657642811 .27688327725 .3801833568 H 17.404482657910 .7634841978 .4162161858 H 17.70979592812 .40653889878 .9783665676 H 16.056499701111 .89327127048 .648885868 H 12.196402801610 .70476237190 .7812764746 H 10.89268572869 .66744631570 .1910035257 H $12.51533206119 .5167265231-0.4956715119$ H $12.35014397317 .1649913841-0.1012736351$ H 10.81094612247 .17360782330 .771678418 H 12.2828303326 .44229947491 .4917408287 H 12.7496390661 .75631870477 .8516851426 H 14.1003651021 .54032247256 .7261564832 H 14.05383545522 .9851737437 .7245559119 H 10.34531689968 .64267119172 .5169169063 H 11.340025056610 .04713085662 .8800787082 Н 20.72919887837 .958311813710 .2451096315 H 20.65505200357 .67073796738 .5029796667 H 21.25951278486 .40461646769 .576763188 H 11.10763163073 .75973088672 .1533648415 H 12.68342146343 .14123055461 .6683285887 H 16.31875432740 .50738817044 .2176012713 H 12.3084417570 .86158928172 .2963170895 H 10.65345391611 .28667815942 .7346356117 H 11.67409177660 .41836144433 .8899995416 H 11.11090333242 .20592073285 .5772147107 H 10.08130691282 .91823588684 .334816501 H 11.29507793613 .91753402995 .1818964526 H 16.02078766432 .88247500939 .1131775911 H 16.50196024611 .77502724067 .81925317 H 16.71237070451 .29638860139 .5164431223 H $15.4345514481-0.82980746439 .0556043519$ H $15.2435060277-0.30444057797 .3800193513$ H $13.8218990254-0.77124773748 .3226194305$ H 10.76262406442 .61224723489 .9350878794 H 10.20079655683 .186155525711 .5024225197 H 11.26178302644 .205022594110 .5379041943 H 13.784741550511 .41795151313 .4467906346 H 12.311247322511 .68057425024 .4074123178 H 13.089721084610 .08015098754 .3635098885 H 12.08218745158 .27516851647 .4431723276 H 10.80219407927 .10446994637 .7237879933 H 13.046812571110 .14648192276 .9108719587 H 12.40283508211 .78798209926 .9070449183 H 13.899969800211 .46460284637 .7781236132 H 8.35132332273 .42694885389 .8556748952 H 8.90247953935 .02999623410 .3752535362 H 14.934640836713 .38181791726 .4429519289 H 13.383185756213 .55127264425 .6132730588 H 14.864818216213 .36605066724 .6719550487 H 10.65820012819 .43642090515 .73566665945 H 9.85520157819 .36524753797 .3204057625 H 9.3601356167 8.2600819017 6.0247117514 H 8.36565770246 .16878271738 .3234644669 
H 7.49453106534 .64553733878 .0588967301 H 7.45683670883 .79641139731 .8812020007 H 8.49737154064 .15889245023 .2599253695 H 8.97988597334 .64574658051 .6167295769 H 8.73152597154 .273062835 .9272265029 H 9.68073595925 .75548686356 .2029279336 H 7.94147856045 .86145891375 .892567609 H 6.5581212525 .7640838473 .1555968375 H 7.0419081385 6.2270702239 1.514737355 Н 6.87180127067 .93080443813 .9385966284 H 8.51233230568 .59914536533 .9095722239 H 6.6365939938 .40297541971 .4736685977 H 8.31510792498 .968799811 .4236993597 Н 7.09605097739 .8675484742 .3374288075

$\mathbf{N}_{2}$

$\mathrm{E}^{0}=-109.5147248 ; \mathrm{G}^{0}=-109.527663$

$\mathrm{E}^{0}{ }_{\mathrm{Et} 2 \mathrm{O}}=-109.5423788 ; \mathrm{G}^{\mathrm{o}} \mathrm{Et2O}=-109.555317$ Charge $=0$; Multiplicity $=1$;

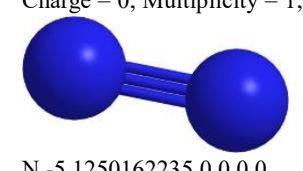

N -5.12501622350 .00 .0$

$\mathrm{N}-4.01486777650 .00 .0$

\section{$\mathbf{E t}_{2} \mathbf{O}$}

$\mathrm{E}^{0}=-233.6322692 ; \mathrm{G}^{0}=-233.525666$;

$\mathrm{E}^{0} \mathrm{Et2O}^{-}=-233.7022263 ; \mathrm{G}^{0} \mathrm{Et2O}=-233.5956231$ Charge $=0 ;$ Multiplicity $=1$

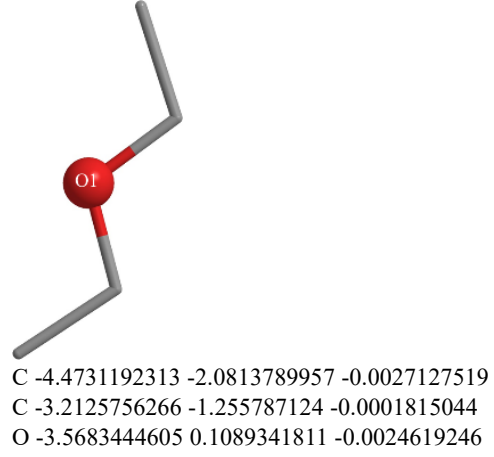

C $-2.43300331640 .9455661962-0.0001859878$ C $-2.89310411722 .38044418-0.0028036854$ H $-4.2411243154-3.1491314512-0.0011022001$ H $-5.0796063655-1.86026998760 .8791115203$ H - $5.0749406986-1.8619453369-0.8881463679$ H - $2.5871154213-1.4865410328-0.882393954$ H - $2.591793022-1.48479798850 .8857828878$ H - 1.8064921950 .73285996080 .885820462 H $-1.80169830970 .7311681682-0.8823713633$ H - $2.04090505353 .0643116701-0.0011575781$ H -3.4988477734 $2.5885986385-0.8882895798$ H -3.5036290936 2.59030192170.878987027

$\mathrm{C}_{2} \mathrm{H}_{4}$

$\mathrm{E}^{\mathrm{o}}=-78.57370621 ; \mathrm{G}^{\mathrm{o}}=-78.54497 ;$

$\mathrm{E}^{0} \mathrm{Et2}_{2}=-78.57515361 ; \mathrm{G}^{0} \mathrm{Et2O}=-78.5464174 ;$ Charge $=0 ;$ Multiplicity $=1$;

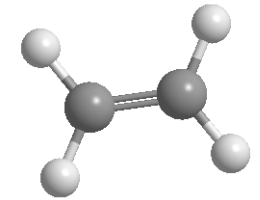

C $-6.2378549492-0.3319770538-0.0000000018$ C -5.08790170230 .33197706180 .0000000018$ H -6.2731990489 -1.41789461190.0000000005 H -7.1959576957 0.18039651560 .0000000005 H -5.0525576218 $1.4178946013-0.0000000005$ H - $4.1297989821-0.180396513-0.0000000005$

$\mathrm{H}_{2}$

$\mathrm{E}^{0}=-1.1702646 ; \mathrm{G}^{0}=-1.171725 ;$

$\mathrm{E}^{0}{ }_{\mathrm{Et} 2 \mathrm{O}}=-1.170006588 ; \mathrm{G}^{0}{ }_{\mathrm{Et} 2 \mathrm{O}}=-1.171466988 ;$ Charge $=0 ;$ Multiplicity $=1$

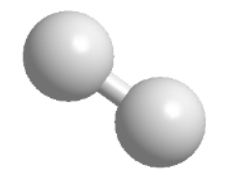

H $-6.6120533502-0.21774746680 .0$

H -7.21565164980 .21774746680 .0$

\section{Cyclic voltammetry}

\section{General information}

Cyclic voltammograms were recorded at room temperature with Princeton Applied Research (PAR) VersaSTAT 3 potentiostat. The working electrode was a glassy carbon disk $\left(\right.$ area $\left.=0.02 \mathrm{~cm}^{2}\right)$, the reference electrode was $\mathrm{Ag} / \mathrm{AgNO}_{3}$ (saturated), and the counter electrode was a platinum wire. 
Cyclic voltammogram

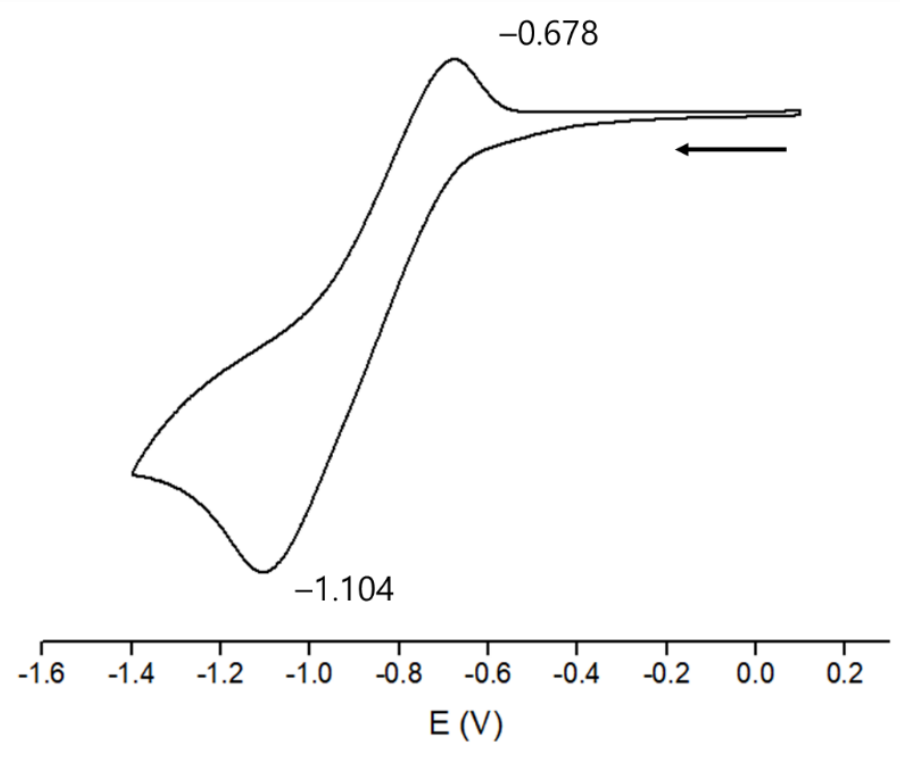

Figure S19. Cyclic voltammogram of $1(2.0 \mathrm{mM})$ in dry and degassed THF with $\mathrm{Bu}_{4} \mathrm{NPF}_{6}(0.1 \mathrm{M})$ as the supporting electrolyte (scan rate $=0.05 \mathrm{~V} / \mathrm{s}$ ). The cyclic voltammogram showed quasi-reversible one-electron redox potential at $\mathrm{E}_{1 / 2}=-0.891 \mathrm{~V}$ versus $\mathrm{Ag} / \mathrm{AgNO}_{3}$ (saturated) electrode, which corresponds to $\mathrm{Ti}(\mathrm{IV})+\mathrm{e}^{-} \rightleftarrows$ $\mathrm{Ti}(\mathrm{III})$. The result was in good agreement with the previous report. ${ }^{22}$

\section{Raman spectra}

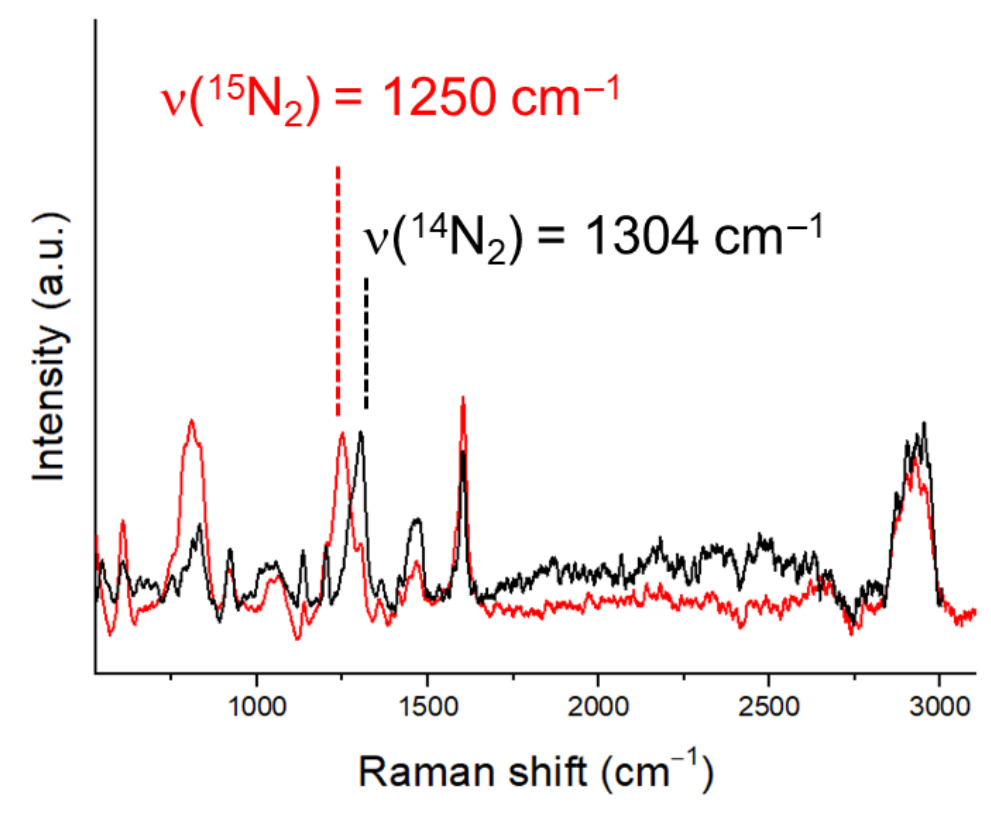

Figure S20. Raman spectra of $\mathbf{3}$ (black trace) and $\mathbf{3}^{\mathbf{1 5}} \mathbf{N}_{2}$ (overlaid with 3; red trace). 


\section{FTIR spectra}

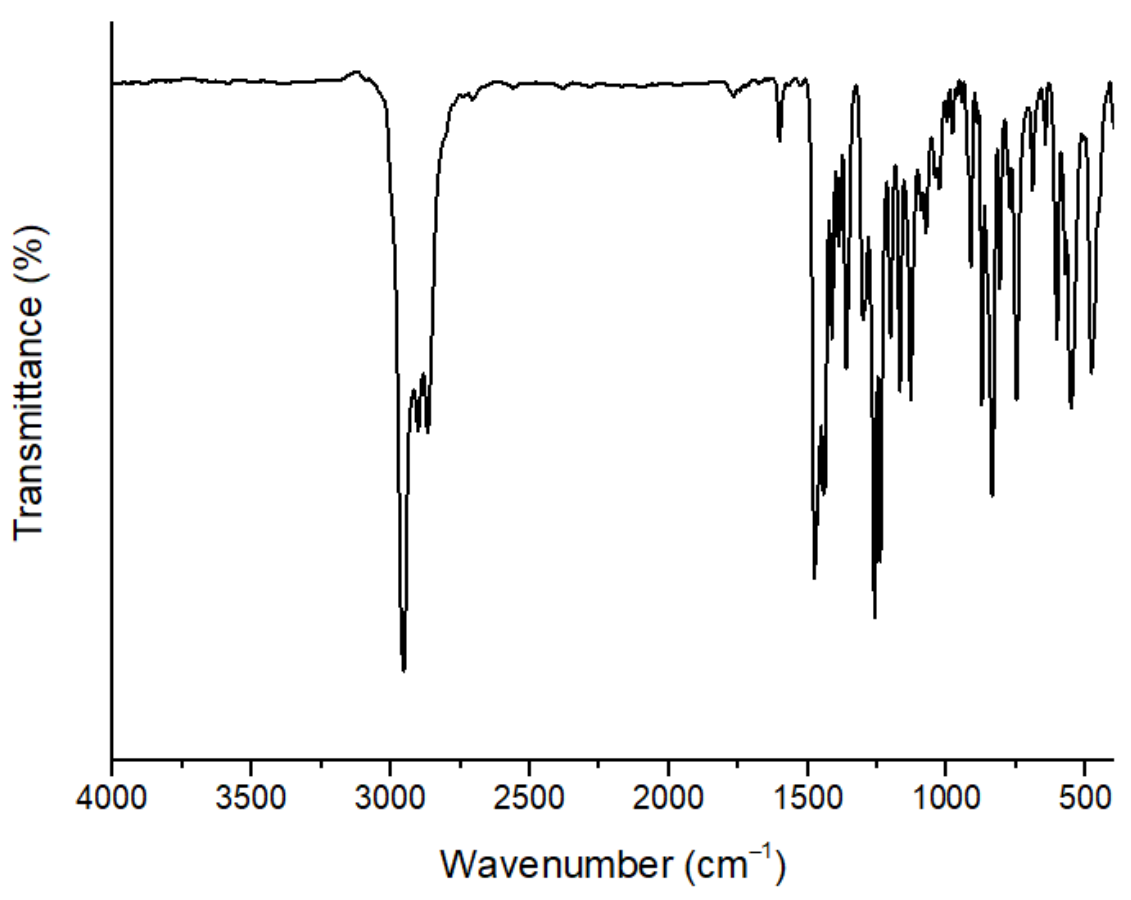

Figure S21. FTIR spectrum of $2\left(\mathrm{KBr}, \mathrm{cm}^{-1}\right)$ : 2954 (s, C-H), 2900 (s, C-H), 2866 (s, C-H), 1602 (s), 1481 (s), 1442 (s), 1361 (s), 1258 (s), 1240 (s), 1172 (s), 1123 (s), 876 (s), 837 (s), 750 (s), 552 (s), 479 (s).

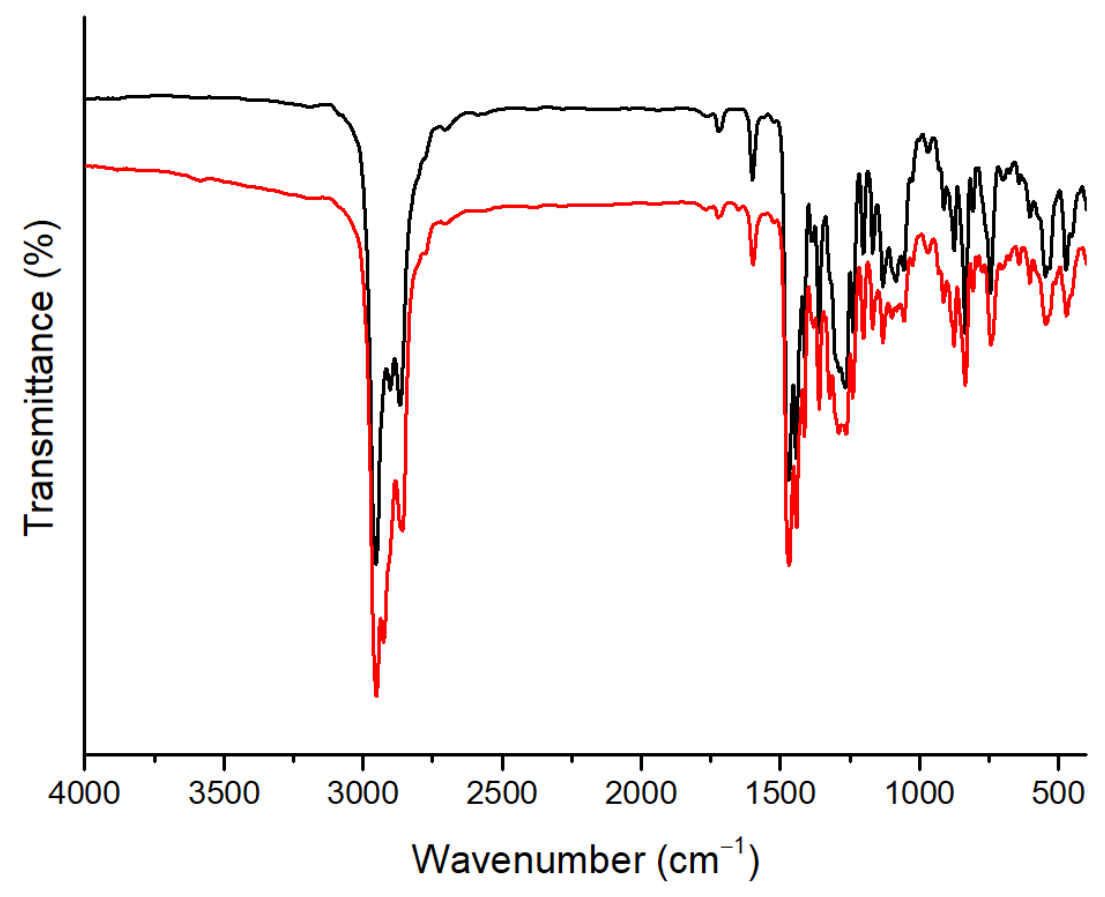

Figure S22. FTIR spectra of $\mathbf{3}$ (black trace) and $\mathbf{3 -}^{\mathbf{1 5}} \mathbf{N}_{2}$ (overlaid with 3; red trace) $\left(\mathrm{KBr}, \mathrm{cm}^{-1}\right)$ : 2958 (s, C-H), 2910 (s, C-H), 2855 (s, C-H), 1717 (s), 1601 (s), 1472 (s), 1430 (s), 1410 (s), 1356 (s), 1260 (s (br)), 1174 (m), 833 (s). 


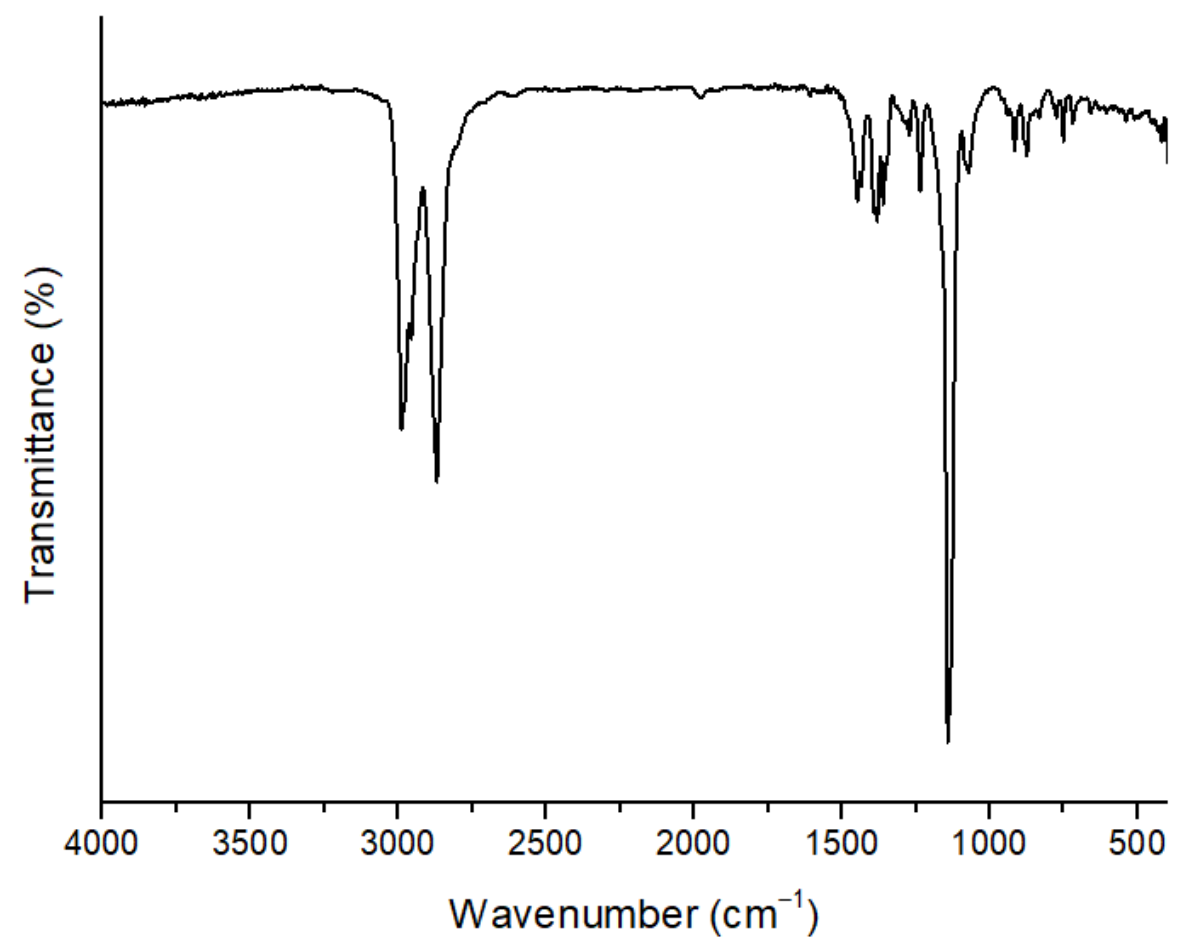

Figure S23. FTIR spectrum of 4 (KBr, cm $\left.{ }^{-1}\right): 2987$ (s, C-H), 2957 (s, C-H), 2869 (s, C-H), 1383 (m), 1237 (s), $1143(\mathrm{~s}), 875(\mathrm{~m}), 748(\mathrm{~m})$.

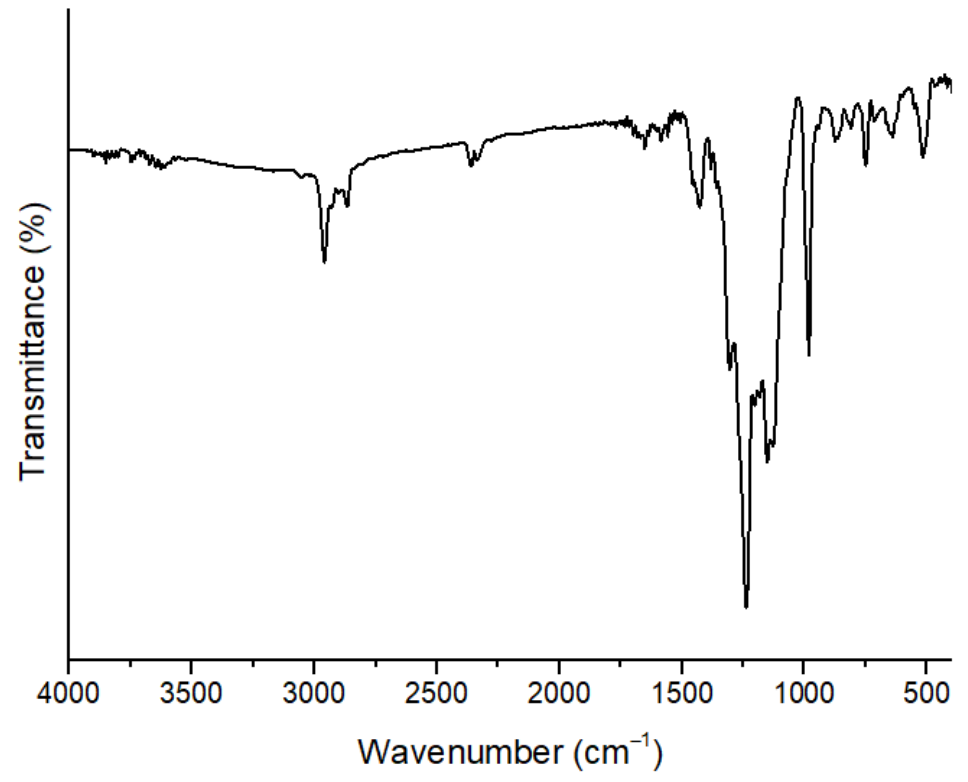

Figure S24. FTIR spectrum of 5 (KBr, cm $\left.{ }^{-1}\right): 2958$ (s, C-H), 2912 (s, C-H), 2865 (s, C-H), 1427 (m), 1232 (s), 1151 (s), 979 (s), 760 (s). 


\section{UV-Vis spectra}

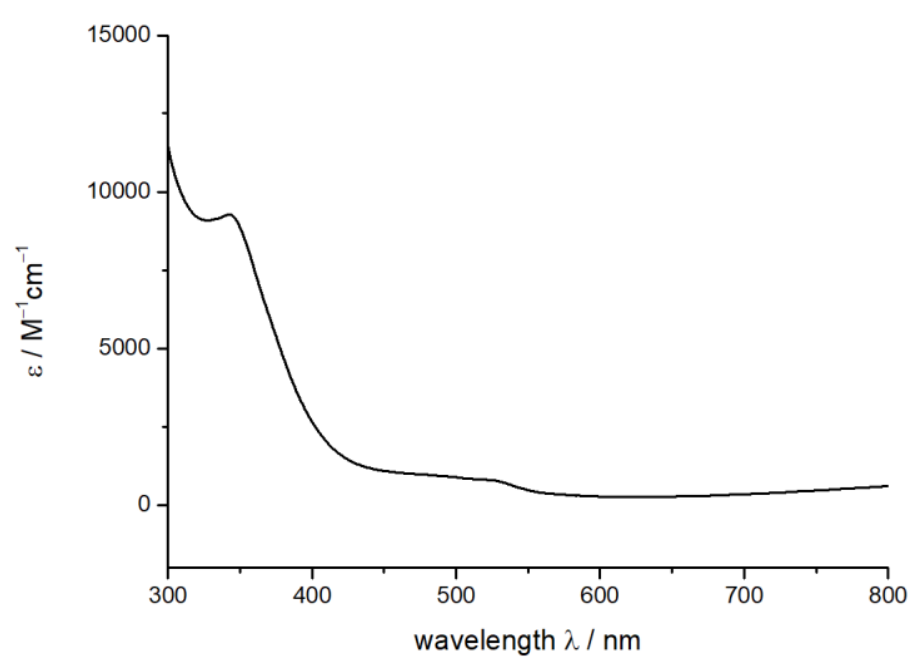

Figure S25. UV-Vis spectrum of of 2 , recorded in benzene. $\left(0.1 \mathrm{mM}, 25{ }^{\circ} \mathrm{C}, \mathrm{nm}\left(\varepsilon=\mathrm{M}^{-1} \mathrm{~cm}^{-1}\right)\right): \lambda(\varepsilon)=343$ (9300), ca. 525 (ca. 771, shoulder).

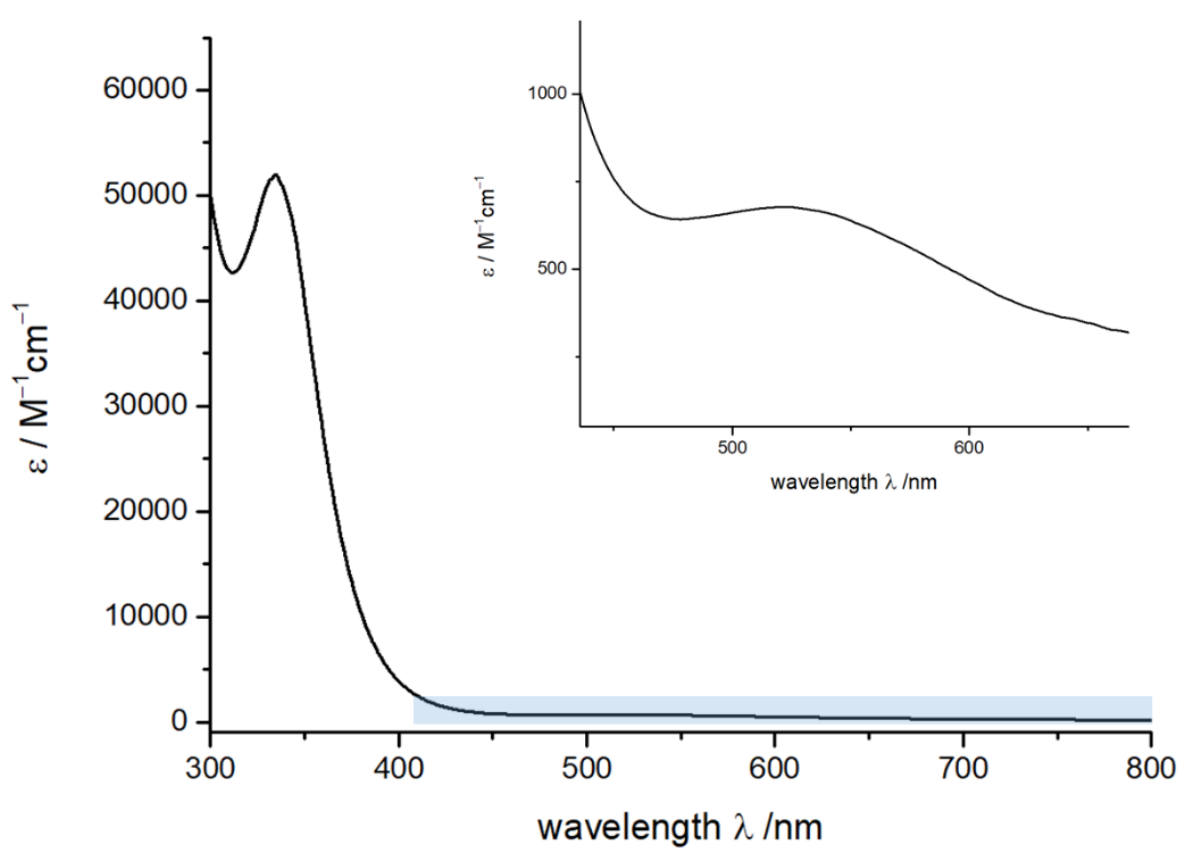

Figure S26. UV-Vis spectrum of of 3 , recorded in toluene. $\left(0.1 \mathrm{mM}, 25{ }^{\circ} \mathrm{C}, \mathrm{nm}\left(\varepsilon=\mathrm{M}^{-1} \mathrm{~cm}^{-1}\right)\right): \lambda(\varepsilon)=292$ (35094), 322 (31064), ca. 555 (ca. 569, shoulder). 


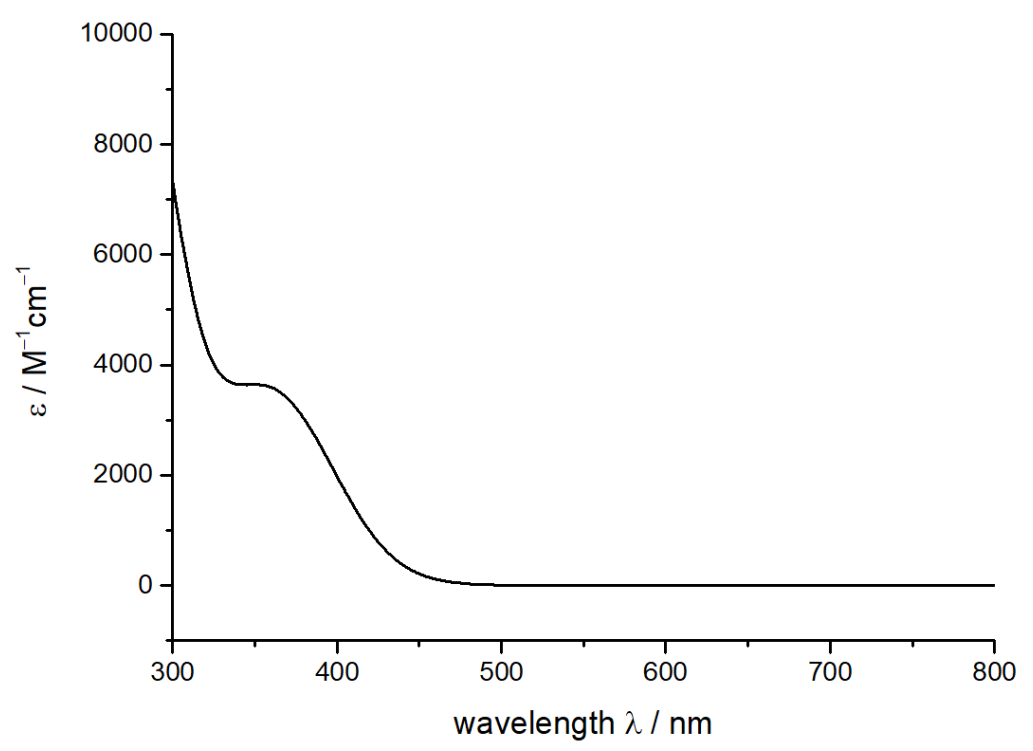

Figure S27. UV-Vis spectrum of of 4 , recorded in benzene. $\left(0.1 \mathrm{mM}, 25^{\circ} \mathrm{C}, \mathrm{nm}\left(\varepsilon=\mathrm{M}^{-1} \mathrm{~cm}^{-1}\right)\right): \lambda(\varepsilon)=\mathrm{ca} .360$ (ca. 3605, shoulder).

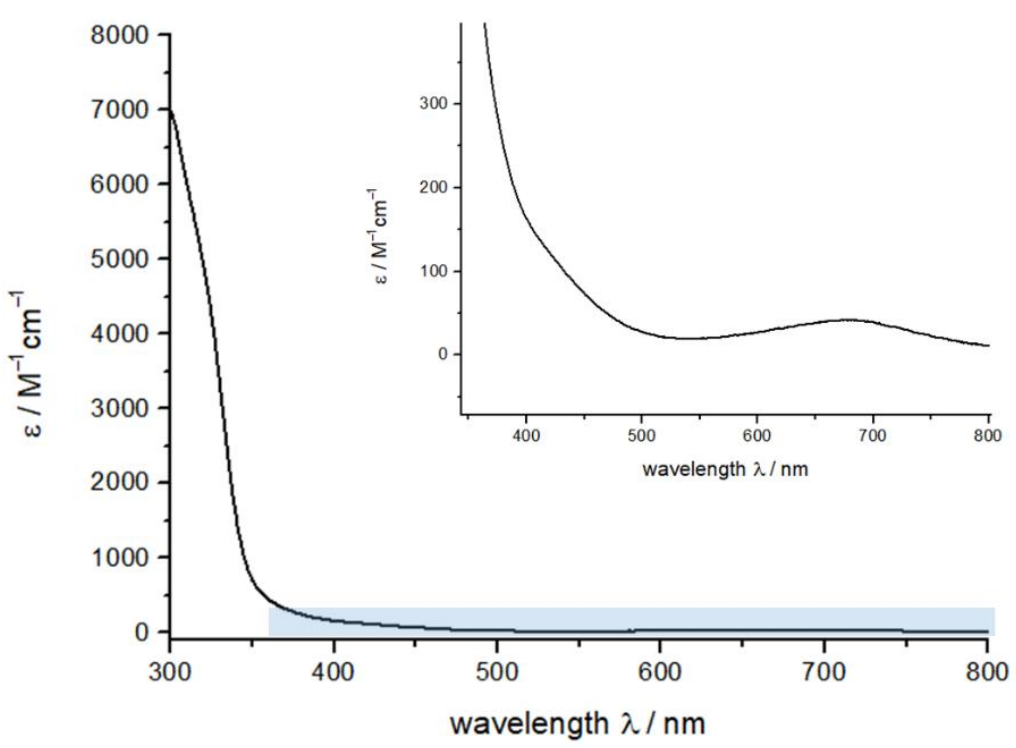

Figure S28. UV-Vis spectrum of of 5, recorded in benzene. $\left(0.1 \mathrm{mM}, 25^{\circ} \mathrm{C}, \mathrm{nm}\left(\varepsilon=\mathrm{M}^{-1} \mathrm{~cm}^{-1}\right)\right): \lambda(\varepsilon)=680(42)$. 
NMR spectra

$\mathbf{L 1 H}_{3}$

${ }^{1} \mathrm{H}$ NMR

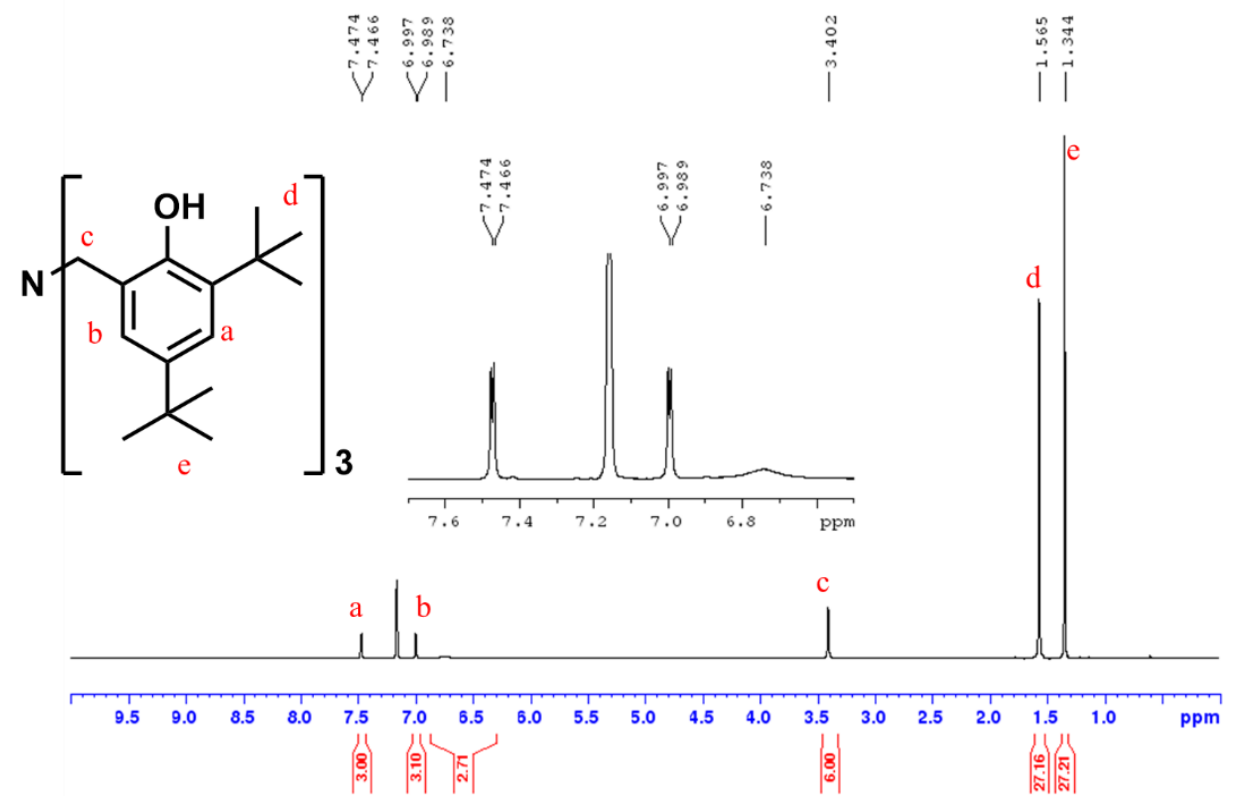

Figure S29. ${ }^{1} \mathrm{H}$ NMR spectrum of $\mathbf{L} 1 \mathrm{H}_{3}$ in $\mathrm{C}_{6} \mathrm{D}_{6}$

${ }^{13} \mathrm{C}$ NMR

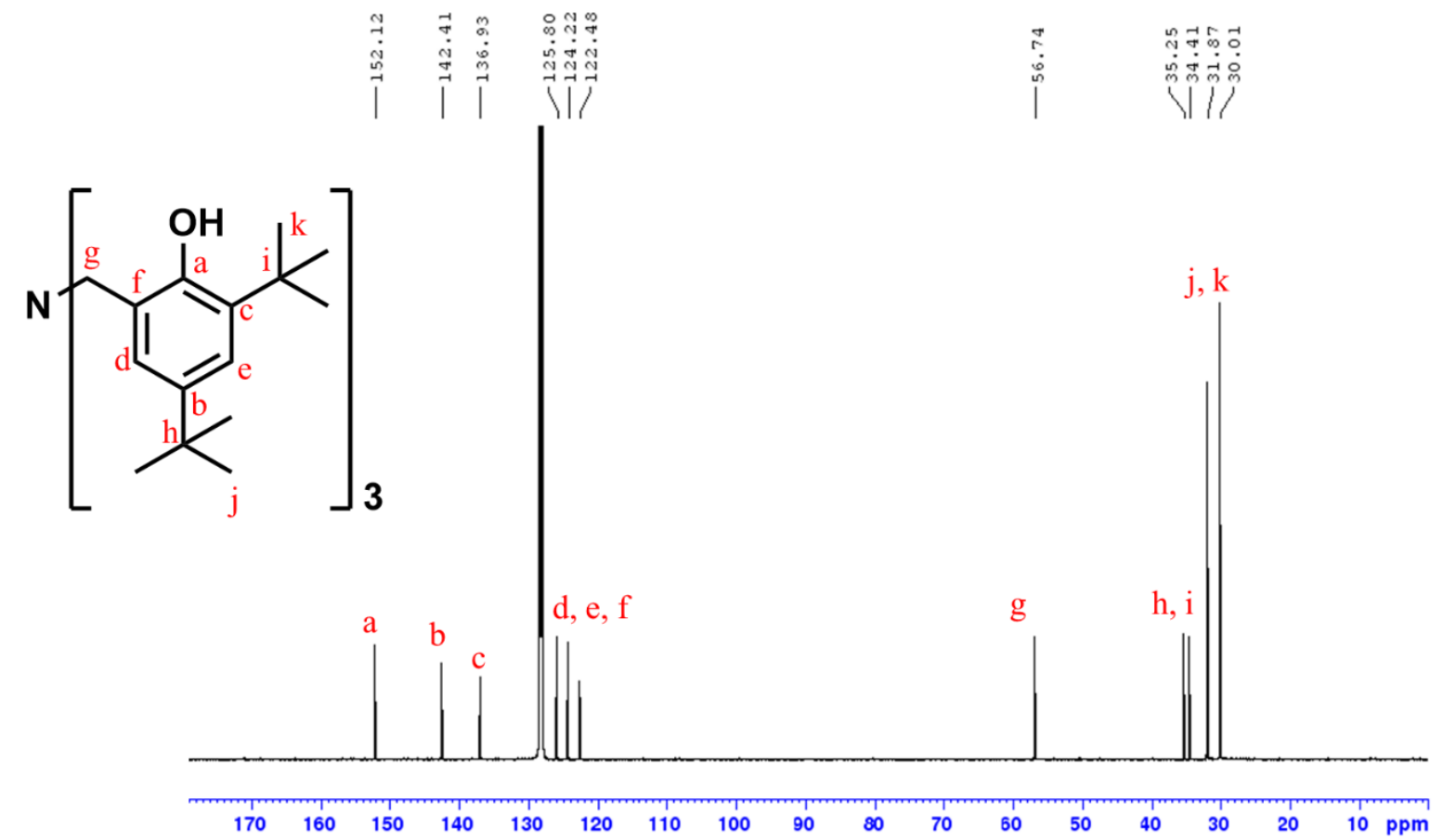

Figure S30. ${ }^{13} \mathrm{C}$ NMR spectrum of $\mathbf{L} 1 \mathrm{H}_{3}$ in $\mathrm{C}_{6} \mathrm{D}_{6}$ 
${ }^{1} \mathrm{H}$ NMR

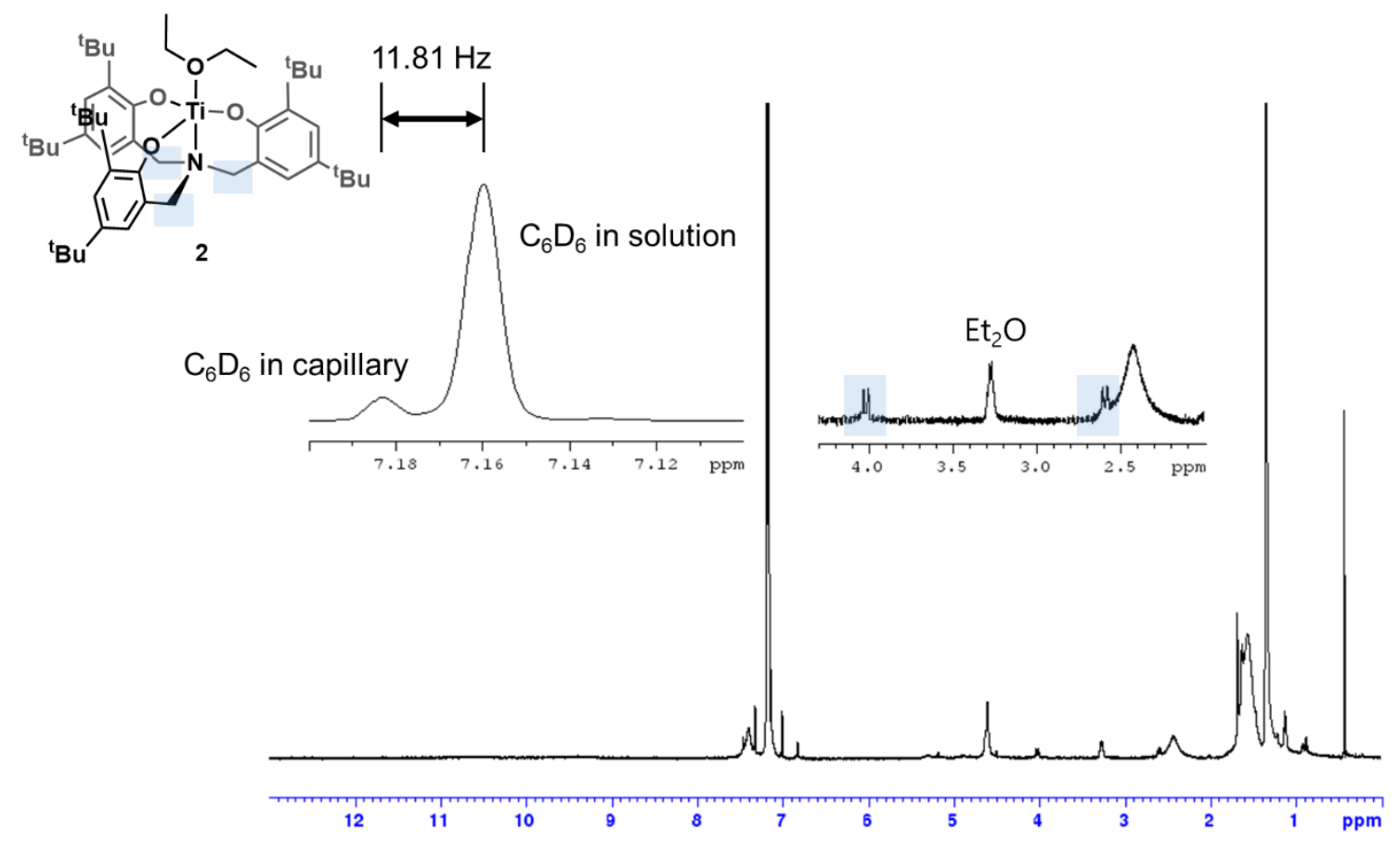

Figure S31. ${ }^{1} \mathrm{H}$ NMR spectrum of 2 in $\mathrm{C}_{6} \mathrm{D}_{6} . \mathrm{C}_{6} \mathrm{D}_{6}$ filled capillary was added to measure magnetic moment. 
${ }^{1} \mathrm{H}$ NMR

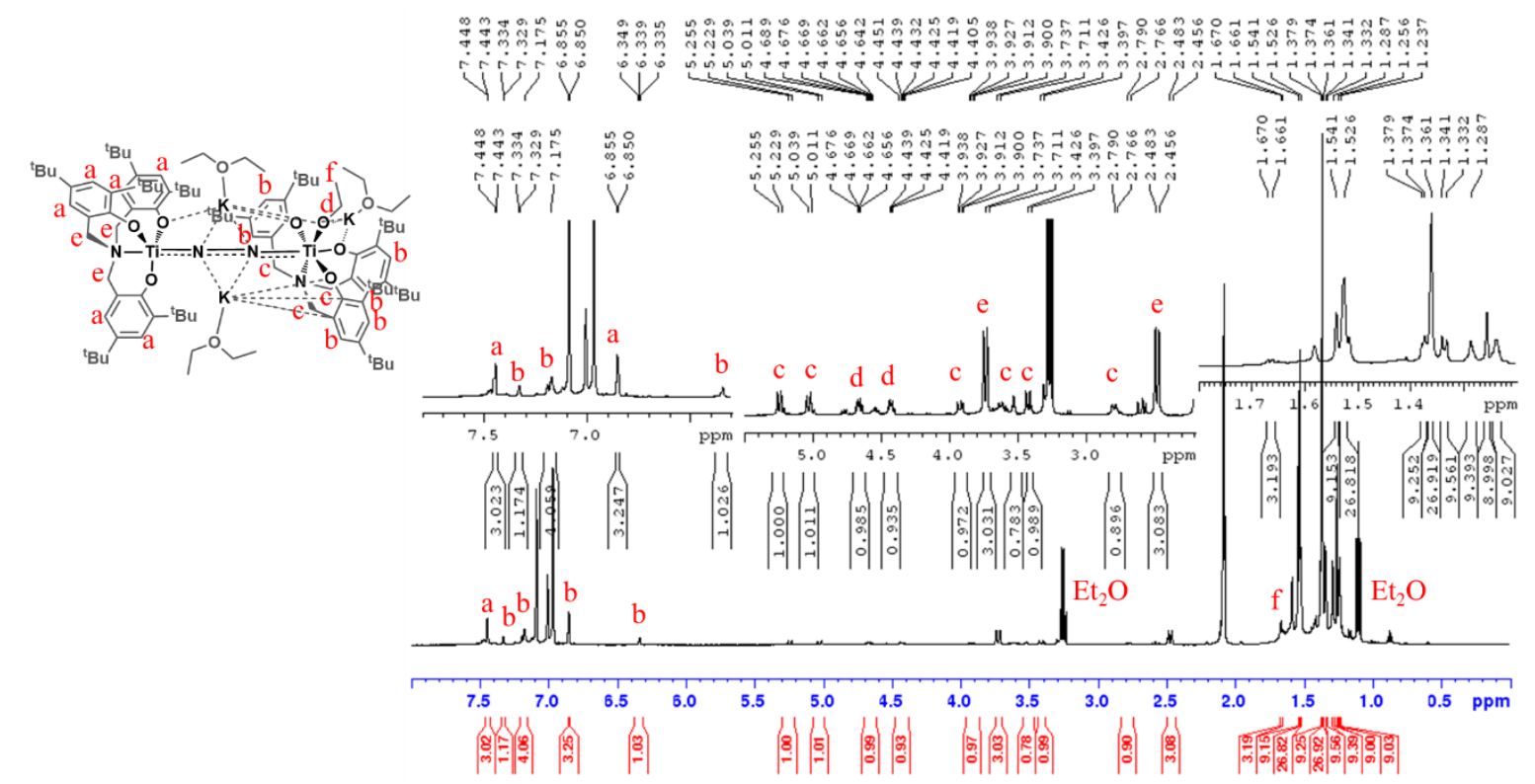

Figure S22. ${ }^{1} \mathrm{H}$ NMR spectrum of 3 in $\mathrm{PhMe}-d_{8}$

${ }^{13} \mathrm{C}$ NMR

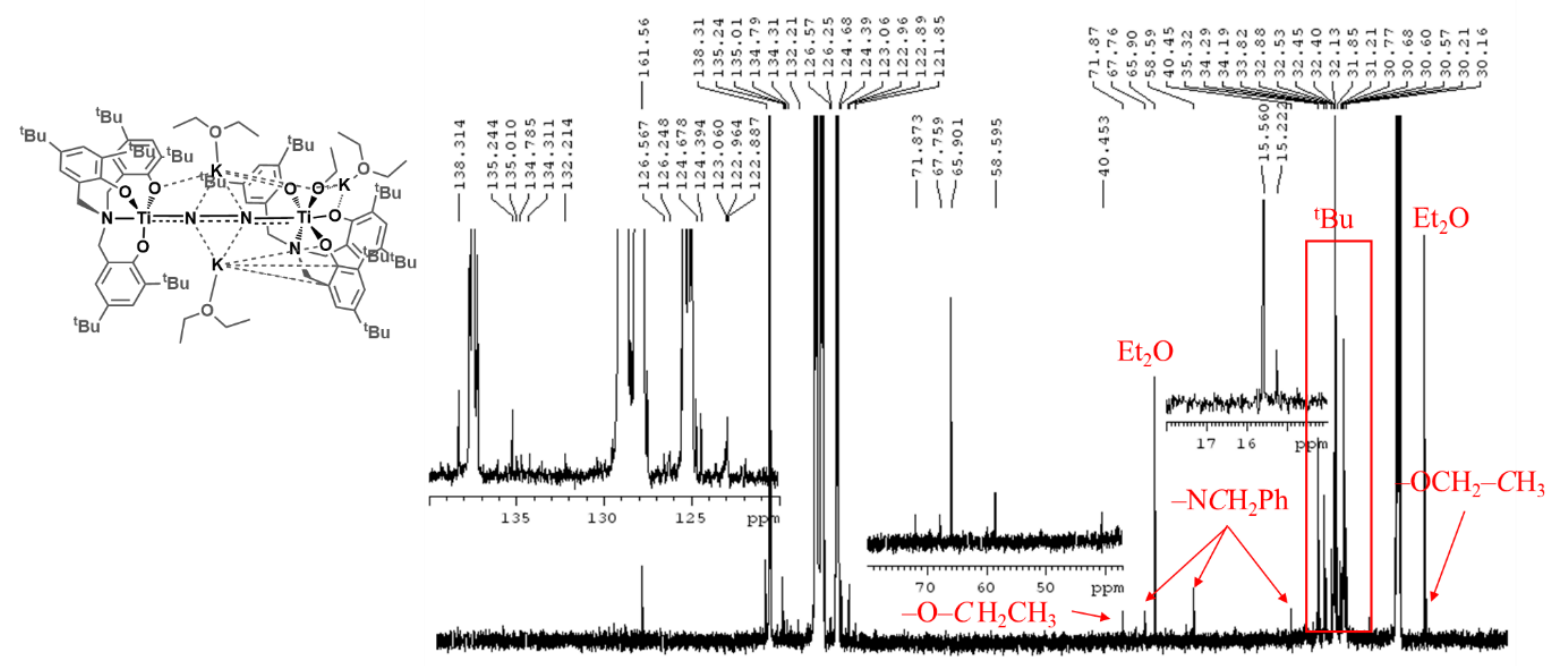

$\begin{array}{lllllllllllllllllll}190 & 130 & 170 & 160 & 150 & 140 & 130 & 120 & 110 & 100 & 90 & 80 & 70 & 60 & 50 & 40 & 30 & 20 & \text { ppm }\end{array}$

Figure S33. ${ }^{13} \mathrm{C}$ NMR spectrum of $\mathbf{3}$ in $\mathrm{PhMe}-d_{8}$ 
${ }^{15} \mathrm{~N}$ NMR

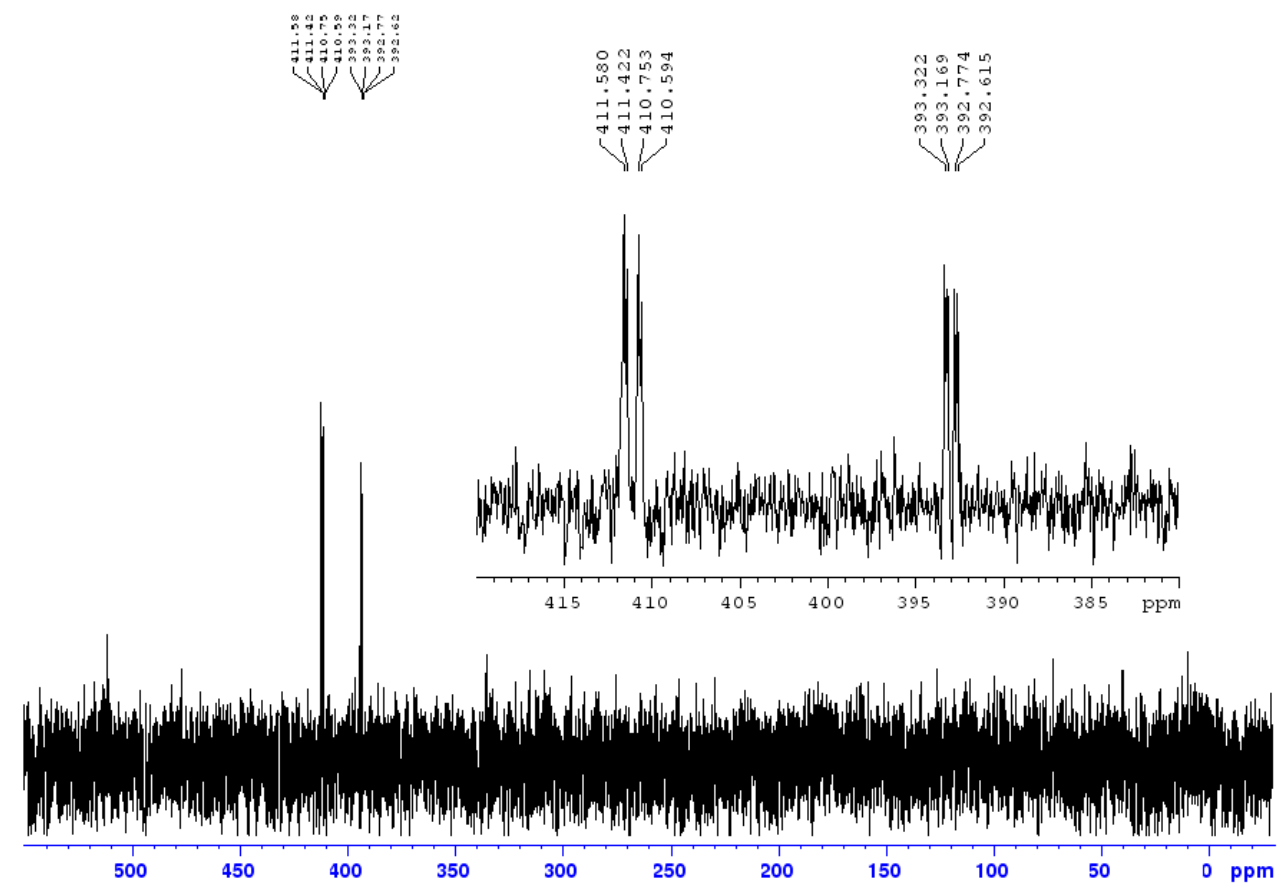

Figure S34. ${ }^{15} \mathrm{~N}$ NMR spectrum of $\mathbf{3 -}^{\mathbf{1 5}} \mathbf{N}_{\mathbf{2}}$ in $\mathrm{PhMe}-d_{8}$ 
${ }^{1} \mathrm{H}$ NMR

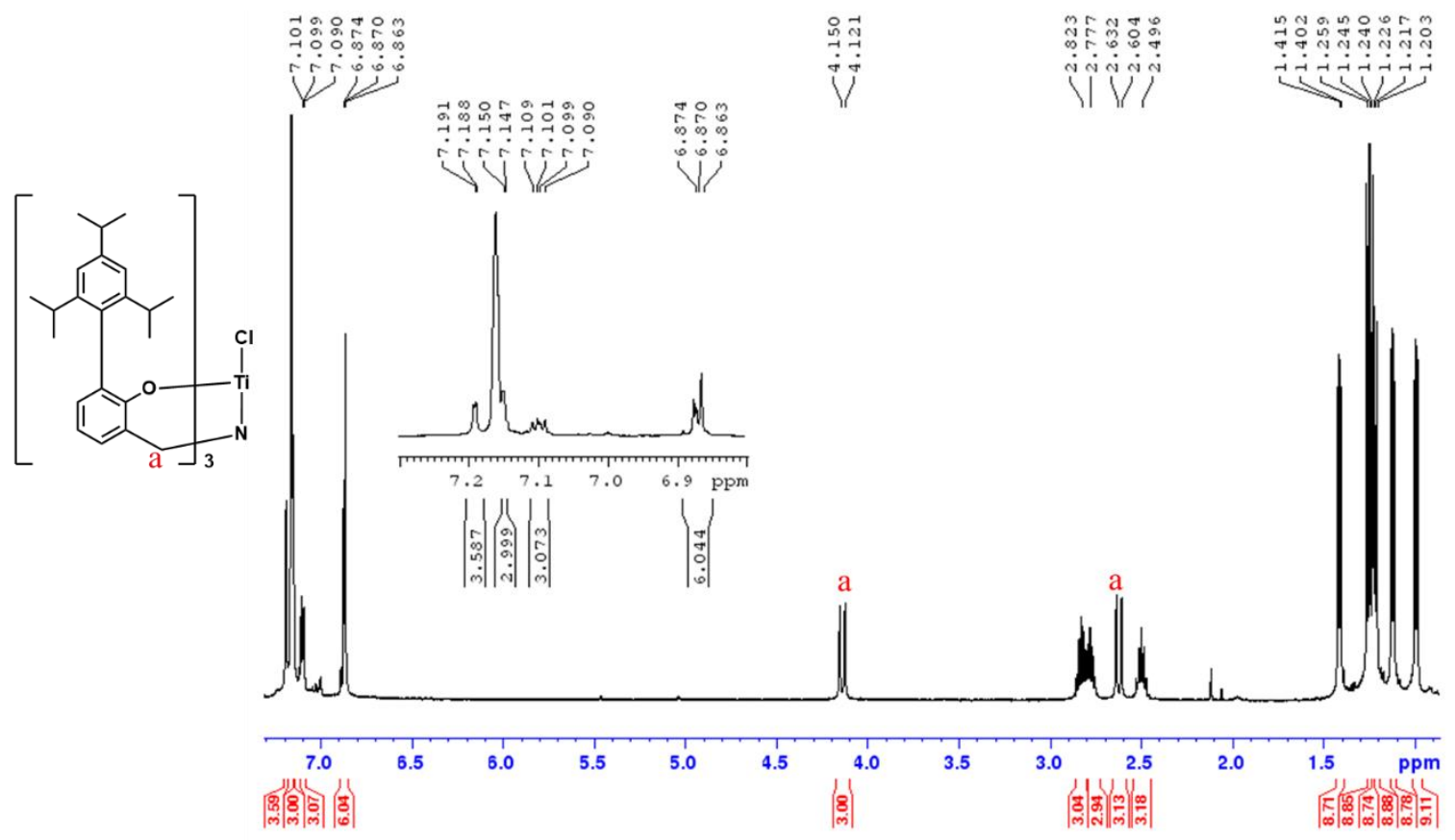

Figure S45. ${ }^{1} \mathrm{H}$ NMR spectrum of 4 in $\mathrm{C}_{6} \mathrm{D}_{6}$

${ }^{13} \mathrm{C}$ NMR

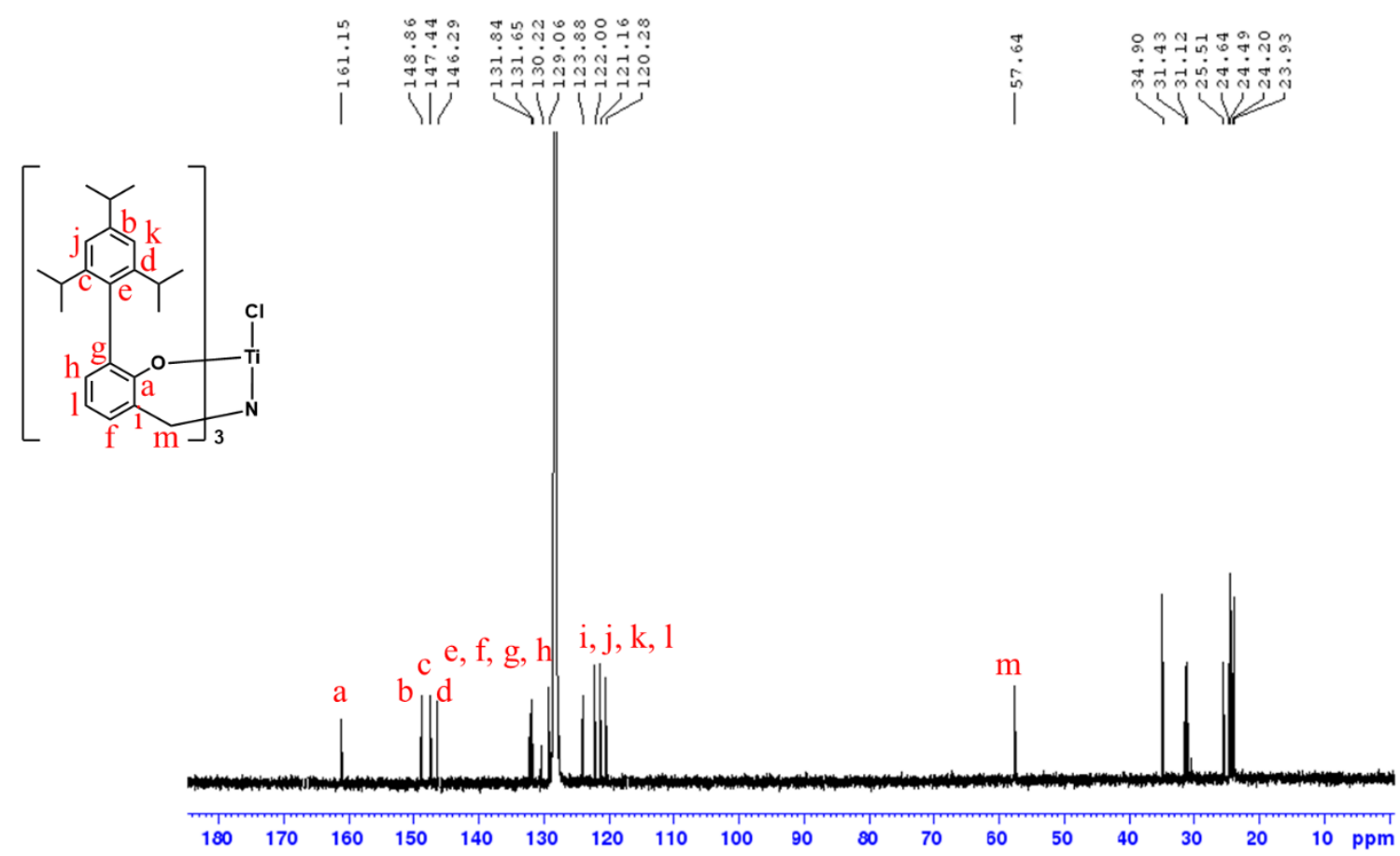

Figure S36. ${ }^{13} \mathrm{C}$ NMR spectrum of 4 in $\mathrm{C}_{6} \mathrm{D}_{6}$ 


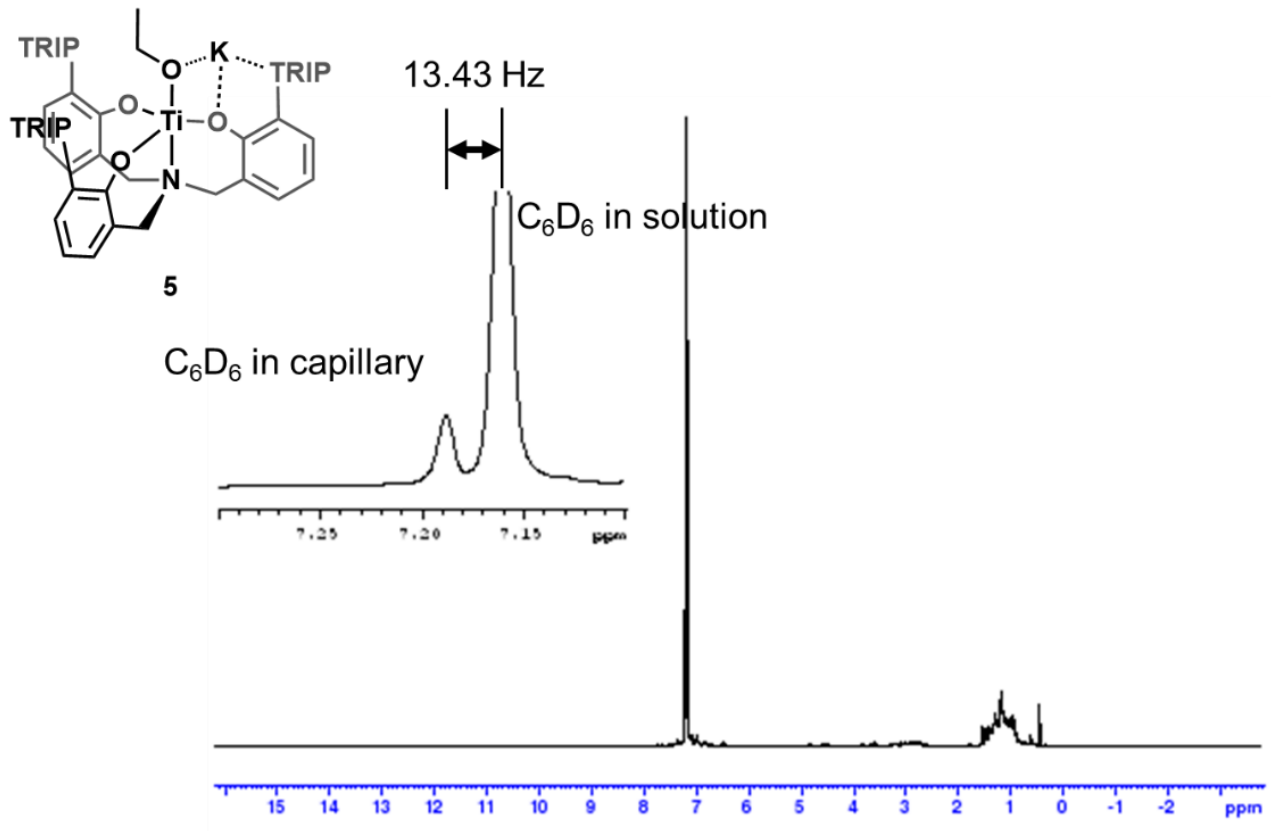

Figure S37. ${ }^{1} \mathrm{H}$ NMR spectrum of 5 in $\mathrm{C}_{6} \mathrm{D}_{6} . \mathrm{C}_{6} \mathrm{D}_{6}$ filled capillary was added to measure magnetic moment.

Table S4. Structurally characterized and reported transition metal dinitrogen dimers with different geometry on each metal center

\begin{tabular}{|c|c|c|c|}
\hline & Geometry & CDS Refcode & Reference \\
\hline Structure & $\begin{array}{c}\text { Trigonal } \\
\text { bipyramidal- } \\
\text { tetrahedral }\end{array}$ & GEBHON & \\
\hline
\end{tabular}




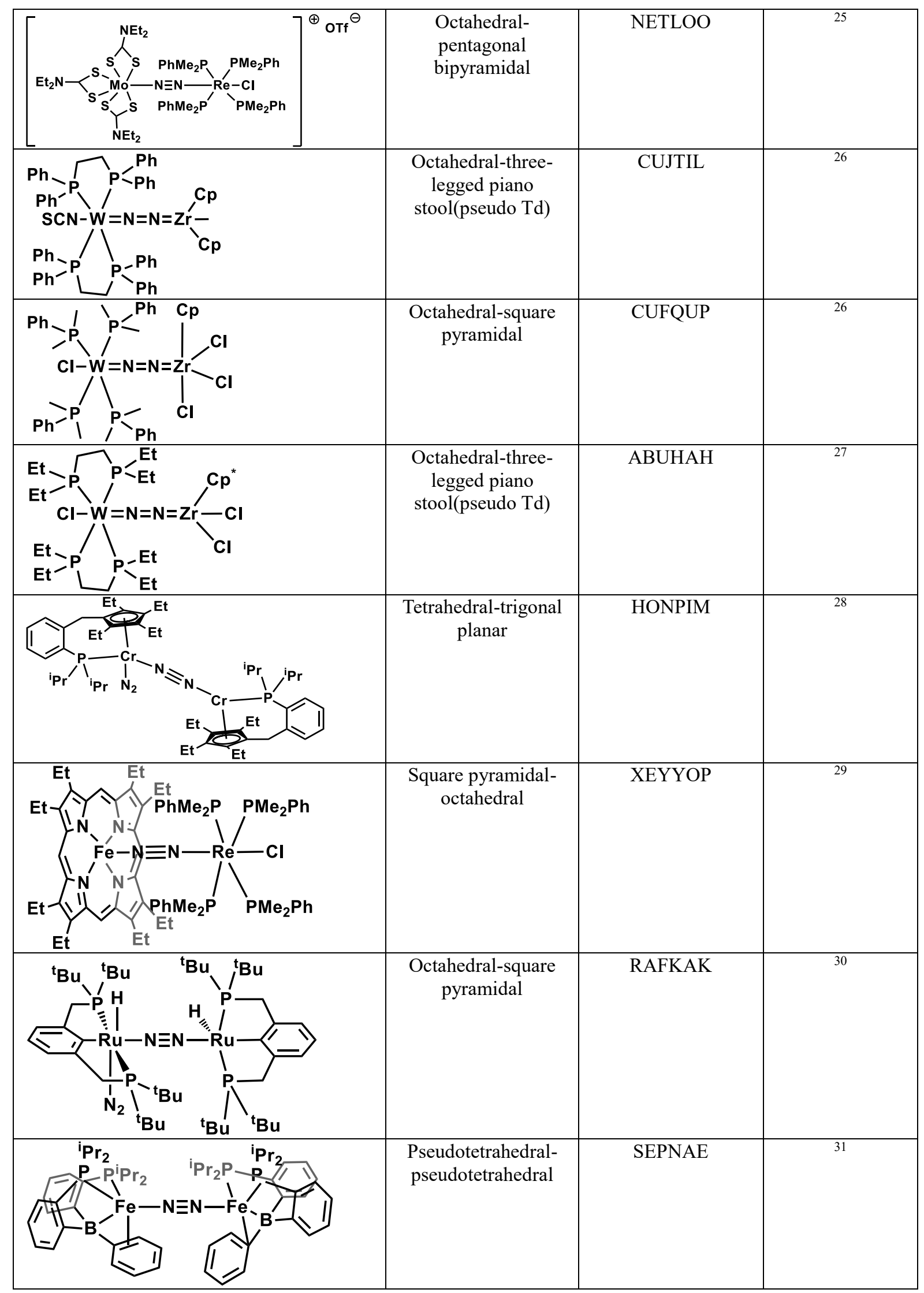




\begin{tabular}{|l|l|l|l|}
\hline & & \\
\end{tabular}

\section{References}

(1) Weatherburn, M. W. Phenol-hypochlorite reaction for determination of ammonia. Anal. Chem. 1967, 39, 971-974.

(2) Doyle, L. R.; Wooles, A. J.; Jenkins, L. C.; Tuna, F.; Mclnnes, E. J. L.; Liddle, S. T. Catalytic Dinitrogen Reduction to Ammonia at a Triamidoamine-Titanium Complex. Angew. Chem. Int. Ed. 2018, 57, 6314-6318.

(3) (a) Brookhart, M.; Grant, B.; Volpe, A. F. [(3,5-(CF3)2C6H3)4B]-[H(OEt2)2]+: a convenient reagent for generation and stabilization of cationic, highly electrophilic organometallic complexes. Organometallics 1992, 11, 3920-3922; (b) Liebing, P.; Pietrasiak, E.; Otth, E.; Kalim, J.; Bornemann, D.; Togni, A. Supramolecular Aggregation of Perfluoroorganyl lodane Reagents in the Solid State and in Solution. Eur. J. Org. Chem. 2018, 2018, 3771-3781.

(4) Nomura, K.; Tewasekson, U.; Takii, Y. Synthesis of Titanium Complexes Containing an Amine Triphenolate Ligand of the Type [TiX $\{(\mathrm{O}-2,4-\mathrm{R} 2 \mathrm{C} 6 \mathrm{H} 2)-6-\mathrm{CH} 2\} 3 \mathrm{~N}]$ and the Ti-Al Heterobimetallic Complexes with AlMe3: Effect of a Terminal Donor Ligand in Ethylene Polymerization. Organometallics 2015, 34, 3272-3281.

(5) Bae, D. Y.; Park, G. S.; Ko, N.; Son, K.-S.; Lee, E. First-row early transition metal complexes with a highly sterically demanding triisopropylphenyl amino triphenolate ligand: synthesis and applications. Dalton Trans. 2019, 48, 9617-9624.

(6) Bergbreiter, D. E.; Killough, J. M. Reactions of potassium-graphite. J. Am. Chem. Soc. 1978, 100, 2126-2134.

(7) (a) Evans, D. F. 400. The determination of the paramagnetic susceptibility of substances in solution by nuclear magnetic resonance. J. Chem. Soc. 1959, 2003-2005; (b) Bain, G. A.; Berry, J. F. Diamagnetic Corrections and Pascal's Constants. J. Chem. Educ. 2008, 85, 532.

(8) (a) Dargaville, T. R.; De Bruyn, P. J.; Lim, A. S. C.; Looney, M. G.; Potter, A. C.; Solomon, D. H.; Zhang, X. Chemistry of novolac resins. II. Reaction of model phenols with hexamethylenetetramine. J. Polym. Sci., Part A: Polym. Chem. 1997, 35, 1389-1398; (b) Kol, M.; Shamis, M.; Goldberg, I.; Goldschmidt, Z.; Alfi, S.; Hayut-Salant, E. Titanium(IV) complexes of trianionic amine triphenolate ligands. Inorg. Chem. Commun. 2001, 4, 177-179.

(9) Wickramasinghe, L. A.; Ogawa, T.; Schrock, R. R.; Müller, P. Reduction of Dinitrogen to Ammonia Catalyzed by Molybdenum Diamido Complexes. J. Am. Chem. Soc. 2017, 139, 9132-9135.

(10) Watt, G. W.; Chrisp, J. D. Spectrophotometric Method for Determination of Hydrazine. Anal. 
Chem. 1952, 24, 2006-2008.

(11) Dolomanov, O. V.; Bourhis, L. J.; Gildea, R. J.; Howard, J. A. K.; Puschmann, H. OLEX2: a complete structure solution, refinement and analysis program. J. Appl. Crystallogr. 2009, 42, 339-341.

(12) Sheldrick, G. SHELXT - Integrated space-group and crystal-structure determination. Acta Crystallogr. A 2015, 71, 3-8.

(13) Sheldrick, G. Crystal structure refinement with SHELXL. Acta Crystallogr. C 2015, 71, 3-8.

(14) Frisch, M. J.; Trucks, G. W.; Schlegel, H. B.; Scuseria, G. E.; Robb, M. A.; Cheeseman, J. R.; Scalmani, G.; Barone, V.; Petersson, G. A.; Nakatsuji, H.; Li, X.; Caricato, M.; Marenich, A. V.; Bloino, J.; Janesko, B. G.; Gomperts, R.; Mennucci, B.; Hratchian, H. P.; Ortiz, J. V.; Izmaylov, A. F.; Sonnenberg, J. L.; Williams; Ding, F.; Lipparini, F.; Egidi, F.; Goings, J.; Peng, B.; Petrone, A.; Henderson, T.; Ranasinghe, D.; Zakrzewski, V. G.; Gao, J.; Rega, N.; Zheng, G.; Liang, W.; Hada, M.; Ehara, M.; Toyota, K.; Fukuda, R.; Hasegawa, J.; Ishida, M.; Nakajima, T.; Honda, Y.; Kitao, O.; Nakai, H.; Vreven, T.; Throssell, K.; Montgomery Jr., J. A.; Peralta, J. E.; Ogliaro, F.; Bearpark, M. J.; Heyd, J. J.; Brothers, E. N.; Kudin, K. N.; Staroverov, V. N.; Keith, T. A.; Kobayashi, R.; Normand, J.; Raghavachari, K.; Rendell, A. P.; Burant, J. C.; lyengar, S. S.; Tomasi, J.; Cossi, M.; Millam, J. M.; Klene, M.; Adamo, C.; Cammi, R.; Ochterski, J. W.; Martin, R. L.; Morokuma, K.; Farkas, O.; Foresman, J. B.; Fox, D. J. Gaussian 16 Rev. C.01, Wallingford, CT, 2016.

(15) Zhao, Y.; Truhlar, D. G. A new local density functional for main-group thermochemistry, transition metal bonding, thermochemical kinetics, and noncovalent interactions. J. Chem. Phys. 2006, 125, 194101.

(16) (a) Becke, A. D. Density-functional thermochemistry. III. The role of exact exchange. J. Chem. Phys. 1993, 98, 5648-5652; (b) Perdew, J. P.; Wang, Y. Accurate and simple analytic representation of the electron-gas correlation energy. Phys. Rev. B 1992, 45, 13244-13249; (c) Marenich, A. V.; Cramer, C. J.; Truhlar, D. G. Universal Solvation Model Based on Solute Electron Density and on a Continuum Model of the Solvent Defined by the Bulk Dielectric Constant and Atomic Surface Tensions. J. Phys. Chem. B 2009, 113, 6378-6396.

(17) (a) Dolg, M.; Wedig, U.; Stoll, H.; Preuss, H. Energy-adjusted ab initio pseudopotentials for the first row transition elements. J. Chem. Phys. 1987, 86, 866-872; (b) Ehlers, A. W.; Böhme, M.; Dapprich, S.; Gobbi, A.; Höllwarth, A.; Jonas, V.; Köhler, K. F.; Stegmann, R.; Veldkamp, A.; Frenking, G. A set of f-polarization functions for pseudo-potential basis sets of the transition metals $\mathrm{Sc} C \mathrm{Cu}, \mathrm{Y} A \mathrm{Ag}$ and La Au. Chem. Phys. Lett. 1993, 208, 111-114.

(18) Höllwarth, A.; Böhme, M.; Dapprich, S.; Ehlers, A. W.; Gobbi, A.; Jonas, V.; Köhler, K. F.; Stegmann, R.; Veldkamp, A.; Frenking, G. A set of d-polarization functions for pseudo-potential basis sets of the main group elements $\mathrm{Al} \mathrm{Bi}$ and f-type polarization functions for $\mathrm{Zn}, \mathrm{Cd}, \mathrm{Hg}$. Chem. Phys. Lett. 1993, 208, 237-240.

(19) Hariharan, P. C.; Pople, J. A. The influence of polarization functions on molecular orbital hydrogenation energies. Theor. Chim. Acta 1973, 28, 213-222. 
(20) Martin, J. M. L.; Sundermann, A. Correlation consistent valence basis sets for use with the Stuttgart-Dresden-Bonn relativistic effective core potentials: The atoms $\mathrm{Ga}-\mathrm{Kr}$ and $\mathrm{In}-\mathrm{Xe}$. J. Chem. Phys. 2001, 114, 3408-3420.

(21) (a) Krishnan, R.; Binkley, J. S.; Seeger, R.; Pople, J. A. Self-consistent molecular orbital methods. XX. A basis set for correlated wave functions. J. Chem. Phys. 1980, 72, 650-654; (b) McLean, A. D.; Chandler, G. S. Contracted Gaussian basis sets for molecular calculations. I. Second row atoms, $Z=11-18$. J. Chem. Phys. 1980, 72, 5639-5648; (c) Frisch, M. J.; Pople, J. A.; Binkley, J. S. Self-consistent molecular orbital methods 25. Supplementary functions for Gaussian basis sets. J. Chem. Phys. 1984, 80, 3265-3269.

(22) Lionetti, D.; Medvecz, A. J.; Ugrinova, V.; Quiroz-Guzman, M.; Noll, B. C.; Brown, S. N. RedoxActive Tripodal Aminetris(aryloxide) Complexes of Titanium(IV). Inorg. Chem. 2010, 49, 4687-4697.

(23) Geri, J. B.; Shanahan, J. P.; Szymczak, N. K. Testing the Push-Pull Hypothesis: Lewis Acid Augmented N2 Activation at Iron. J. Am. Chem. Soc. 2017, 139, 5952-5956.

(24) Cozzolino, A. F.; Silvia, J. S.; Lopez, N.; Cummins, C. C. Experimental and computational studies on the formation of cyanate from early metal terminal nitrido ligands and carbon monoxide. Dalton Trans. 2014, 43, 4639-4652.

(25) Seymore, S. B.; Brown, S. N. Kinetic Effects in Heterometallic Dinitrogen Cleavage. Inorg. Chem. 2006, 45, 9540-9550.

(26) Ishino, H.; Nagano, T.; Kuwata, S.; Yokobayashi, Y.; Ishii, Y.; Hidai, M.; Mizobe, Y. Syntheses, Structures, and Reactivities of Heterobimetallic Bridging Dinitrogen Complexes Containing Group 6 and Group 4 or 5 Transition Metals1. Organometallics 2001, 20, 188-198.

(27) Ishino, H.; Takemoto, S.; Hirata, K.; Kanaizuka, Y.; Hidai, M.; Nabika, M.; Seki, Y.; Miyatake, T.; Suzuki, N. Olefin Polymerization Catalyzed by Titanium-Tungsten Heterobimetallic Dinitrogen Complexes1. Organometallics 2004, 23, 4544-4546.

(28) Li, J.; Yin, J.; Wang, G.-X.; Yin, Z.-B.; Zhang, W.-X.; Xi, Z. Synthesis and reactivity of asymmetric $\mathrm{Cr}$ (i) dinitrogen complexes supported by cyclopentadienyl-phosphine ligands. Chem. Commun. 2019, 55, 9641-9644.

(29) Zhang, Q.-F.; Chim, J. L. C.; Lai, W.; Wong, W.-T.; Leung, W.-H. Bridged Dinitrogen Complexes of Iron and Chromium Porphyrins. Inorg. Chem. 2001, 40, 2470-2471.

(30) Gusev, D. G.; Dolgushin, F. M.; Antipin, M. Y. Hydride, Borohydride, and Dinitrogen Pincer Complexes of Ruthenium. Organometallics 2000, 19, 3429-3434.

(31) Suess, D. L. M.; Peters, J. C. H-H and Si-H Bond Addition to Fe $\equiv N N R 2$ Intermediates Derived from N2. J. Am. Chem. Soc. 2013, 135, 4938-4941.

(32) (a) Fryzuk, M. D.; Johnson, S. A.; Rettig, S. J. New Mode of Coordination for the Dinitrogen Ligand: A Dinuclear Tantalum Complex with a Bridging N2 Unit That Is Both Side-On and End-On. J. Am. Chem. Soc. 1998, 120, 11024-11025; (b) Studt, F.; MacKay, B. A.; Johnson, S. A.; Patrick, B. O.; Fryzuk, M. D.; Tuczek, F. Lewis Adducts of the Side-On End-On Dinitrogen-Bridged Complex 
[\{(NPN)Ta\}2 $(\mu-H) 2(\mu-\eta 1: \eta 2-N 2)]$ with AlMe3, GaMe3, and B(C6F5)3: Synthesis, Structure, and Spectroscopic Properties. Chem. Eur. J. 2005, 11, 604-618. 